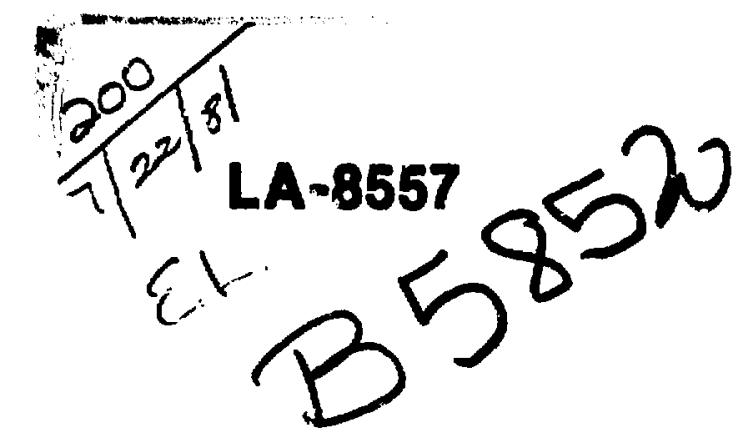

高 


\title{
LA-8557
}

UC-21

Issued: May 1981

\section{Models and Analyses for \\ Inertial-Confinement Fusion-Reactor Studies}

\author{
Ihor O. Bohachevsky
}
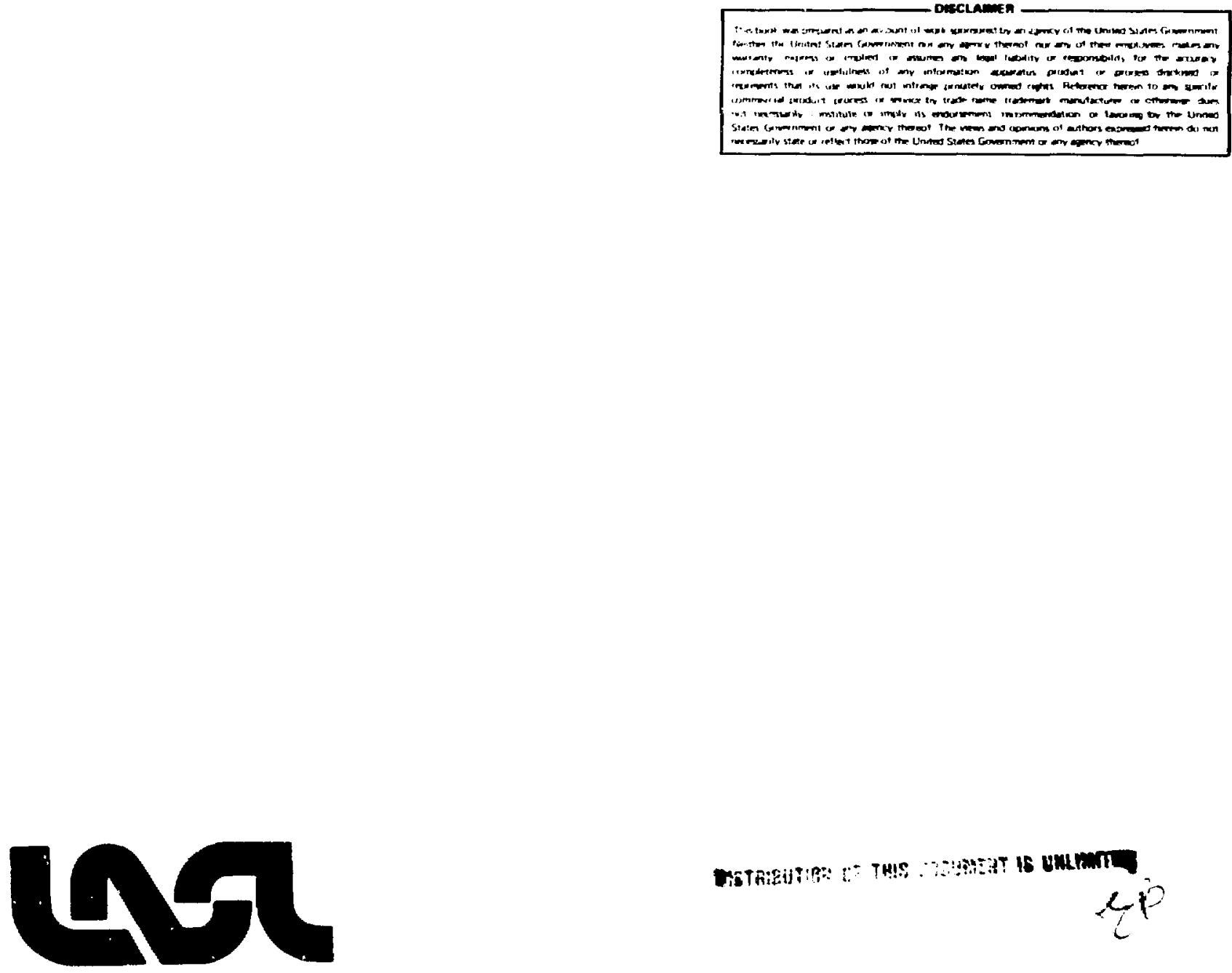

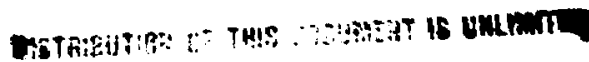<smiles>C1CCC(CC2CC2)C1</smiles> 


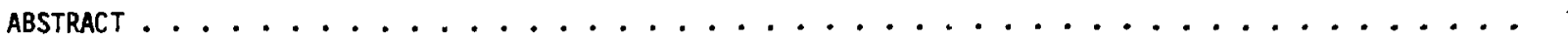

I. GENERAL INERTIAL CONFINEMENT FUSION (ICF) REACTOR DESIGN CONSIDERATIONS . . . . . . . . 1

A. ICF Reactor System Requirements ........................... I

B. Reactor System Components ............................... 2

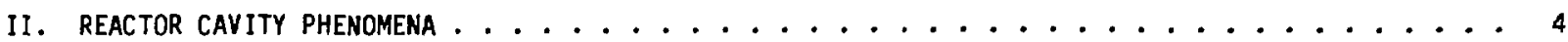

A. General Considerations .............................. 4

B. Cavity Phenonena Modeling ............................ 5

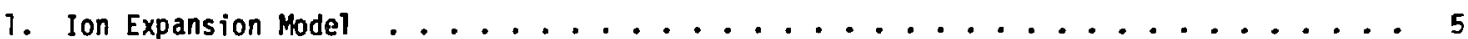

2. Ion Expansion, Computational Procedure .................... 8

3. Ion Expansion, Illustrative Results ...................... g

4. Radiation Effects, Fireball and Blast Wave . . . . . . . . . . . . . . 13

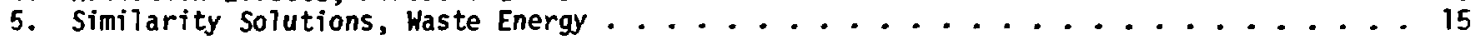

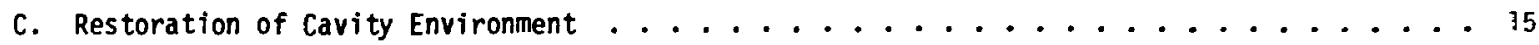

1. Reservoir Exhaust ........................... 16

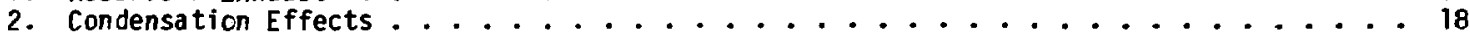

III. FIRST-WALL EFFECTS

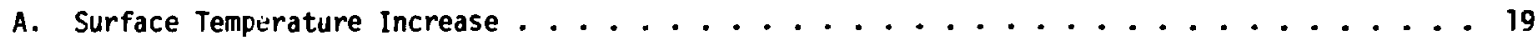

1. Surface flux Model . . . . . . . . . . . . . . . . . . . . 19

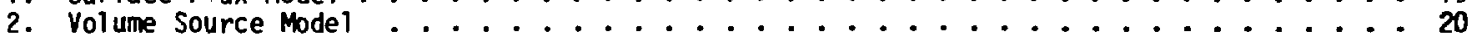

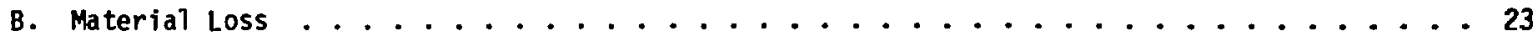

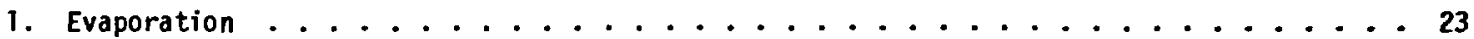

2. Sputtering Erosion in Inertial Confinement fusion Reactors . . . . . . . . . 24

a. Introduction ............................ 24

b. Generat Description ........................ 24

c. Dependence of Sputtering and Its Approximation .............. 25

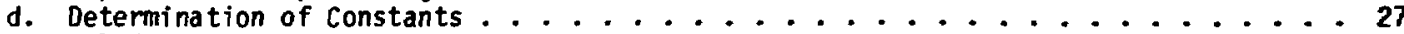

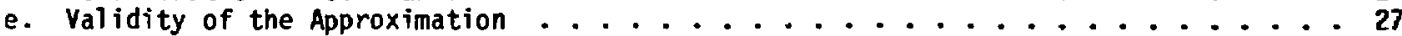

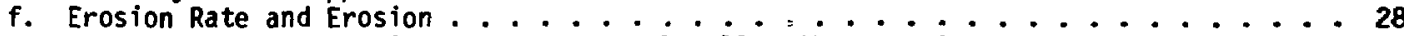

g. Dependence of Sputtering Erosion on Fuel-Pellet Characteristics . . . . . . 29

h. Erosion Estinates ........................ 31

i. Low-Energy Regime. . . . . . . . . . . . . . . . . . . . . 31

j. Intermediate-Energy Regime . . . . . . . . ............ 32

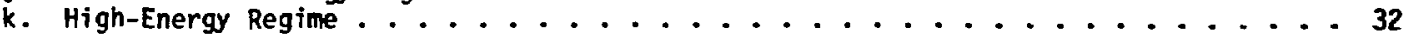

1. Dependence of Erosion Rate on Ion-Beam Characteristics ............ 33

IV. ICF REACTOR NEUTRONICS . . . . . . . . . . . . . . . . . . . . 34

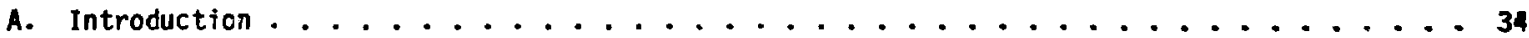

B. Neutron Transport ............................... 34

C. Energy Deposition and Blanket Response . . . . . . . . . . . . . . . . 35

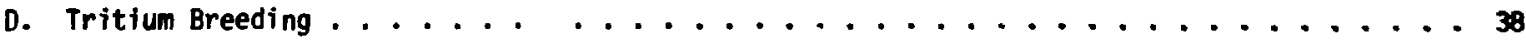

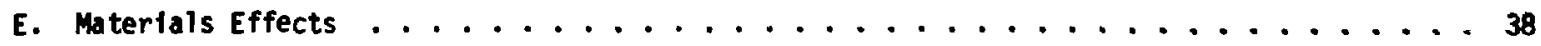

1. General Considerations . . . . . . . . . . . . . . . . . . . 38

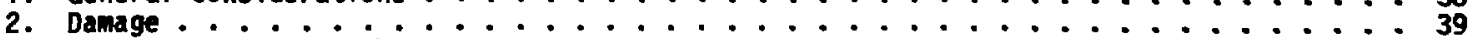

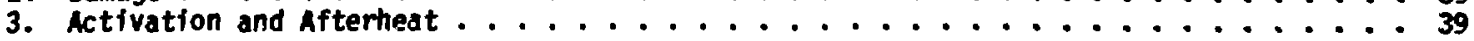

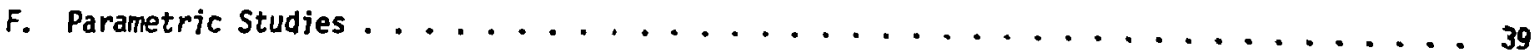




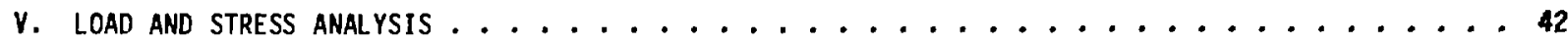

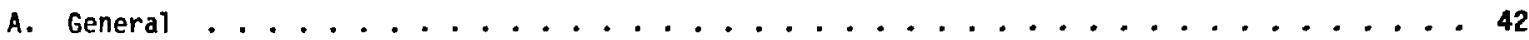

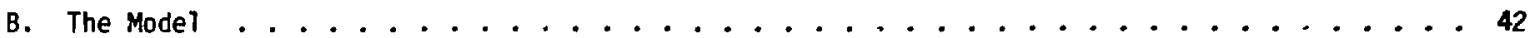

C. Impulse Generation . . . . . . . . . . . . . . . . . . . . . . . 43

1. Evaporation Recoil .............................443

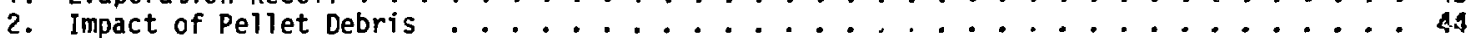

3. Blast Wave Reflection ............................ 44

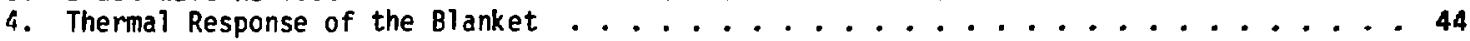

D. Elastic Stability ............................. 45

E. Sample Results ...............................46

F. Hydrodynamic Coupting ................................46

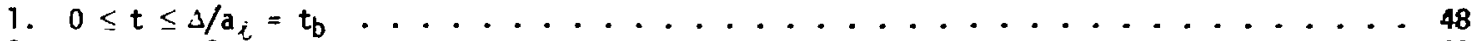

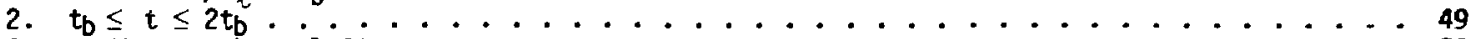

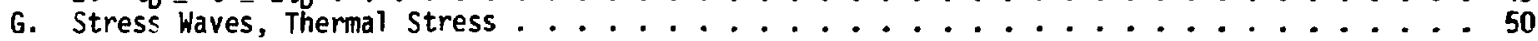

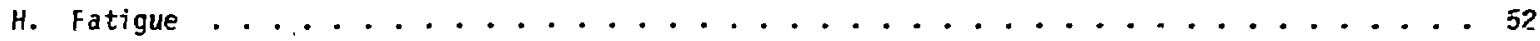

I. Concluding Remarks .............................. 52

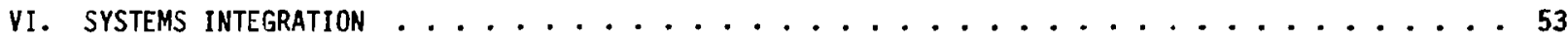

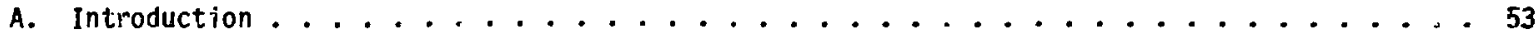

B. Systems Integration Procedure ... . . . . . . . . . . . . . . . . . 54

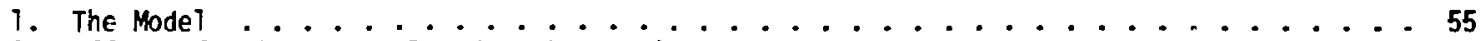

2. Effect of Gain Curve, Boundary Constraints .................. 56

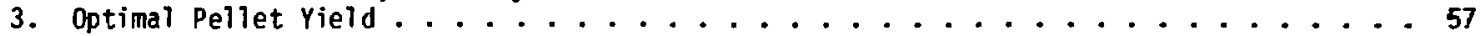

4. Reactor Configuration ....................... 58

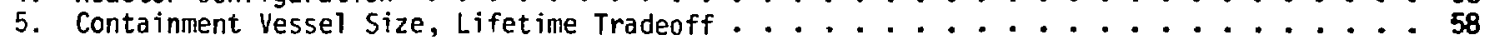

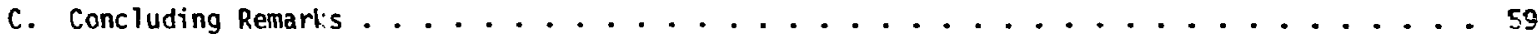

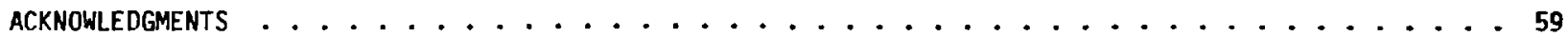

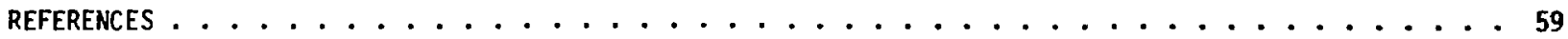


Studies of the technical and economic feasibility of inertial confinement fusion (ICF) reactors for electric power generation and for other commercfal applications have been conducted at the Los Alamos National Laboratory for the past decade. Although prevfously in the Laser Division, the group conducting these investigations is now a part of the Analysis and Assessment Division of the Laboratory.

During the course of these studies, several specific reactor concepts were conceived and developed, many mathematical models were constructed, and numerous analyses were performed. Results and descriptions of this work are contained in technical papers, reports, summaries of presentations, and unpublished memoranda. The present report evolved from a desire to combine these individual contributions into one accessible reference that also would serve as a tutorial introduction to ICF reactor systems studies.

In selecting material for this report, preference was given to explicit analytical results that provide insights into trends and parametric dependencies, rather than to detailed numerical calculations that usually are pertinent oniy to specific concepts or designs. Inadequacies inevitably exist in the treatment of some areas. Constructive criticism of the report will be welcomed in anticipation of improved and more complete revisions.

The report is organized to facilitate a logical progression of conceptualization and analysis leading to preliminary reactor designs. It begins with a discussion of fuel-pellet microexplosions and the characteristics of their emissions, then proceeds through the interactions of these emissions with the cavity background medium and structure to load and stress analyses. The details are presented in six sections.

Section I introduces general ICF reactor design considerations and sumarizes requirements for preliminary specification of reactor conceptual designs.

In Sec. II, the phencmena that develop inside reactor cavities due to interactions between fusionpellet microexplosion products and cavity residual gases are discussed. Also discussed in this section are requirements for restoring the cavity environment to conditions permitting a successive pellet microexplosion.

In Sec. III, methods for estimating temperature increases and material losses induced at the inside surface of the reactor cavity wall by energy and particles released during fusion-pellet burn are presented.

In Sec. IV, the effects of neutrons emitted from the pellet are characterized and methocis for analyzing these effects are indicated.

In Sec. $V$, modeling and analysis of loads and stresses in ICF reactor vessels are discussed and general results pertaining to the dependencies of these quantities on various engineering design parameter are presented.

Sec. VI consists of general remarks pertaining to ICF reactor and power plant systems integrations. Letalled computer calculations are onitted in favor of simple analytic models that my be used to define preliminary reactor configurations and to estimate the sensitivities of their characteristics to changes in values of design parameters. Typical results are included to illustrate the approach. 
MODELS AND ANALYSES FOR

INERTIAL CONFINEMENT FUSION REACTOR STUDIES

by

Ihor 0 . Bohachersky

\section{ABSTRACT}

This report describes models and analyses devised at Los Alamos Nationa) Laboratory to determine the technical characteristics of differe 't inertial confinement fusion (ICF) reactor elements required for componer integration into a functional unit. We emphasize the generic properties or the different elements rather than specific designs. The topics discussed are general ICF reactor design considerations; reactor cavity phenomena, including the restoration of interpulse ambient conditions; first-wall temperature increases and material losses; reactor neutronics and hydrodynamic blanket response to neutron energy deposition; and analyses of loads and stresses in the reactor vessel walls, including remarks about the generation and propagation of very short wavelength stress waves. A discussion of analytic approaches useful in integrations and optimizations of ICF reactor systems concludes the report.

I. GENERAL INERTIAL CONFINEMENT FUSION (ICF) REACTOR DESIGN CONSIDERATIONS

A. ICF Reactor System Requirements

ICF is the approach to harnessing nuclear fusion energy in which the reacting fuel is held together (confined) by the inertia of its mass and, to some degree, by the inertia of the mass of the surrounding material (tamper). The energy liberated by the fusion of two atoms is very large. When the total energy released must be controlled (that is, when it is in the range 100 to $1000 \mathrm{MJ}$ per pulse), the mass of the fuel reacting at one time must be small. This means the inertial confinement time is short (on the order of $\left.10^{-9} \mathrm{~s}\right)$; consequently, a substantial fraction (> 10x) of the fuel will react only if it is initialiy compressed to a density between 50 and $500 \mathrm{~g} / \mathrm{cm}^{3}$. In ICF, the fuel is compressed by depositing a large amount of energy on or near the surface of $\bar{a}$ spherical pellet. The resulting temperature rise evaporates the surface material, and vaporization produces a reaction force (analogous to the rocket action) that compresses the pellet.

The fuel temperature must be increased into the range of thermonuclear reaction values. Because these values are lowest for the fusion of deuterium and tritium (D-T; 10 to $60 \mathrm{keV}$ or $10^{8}$ to $\left.6 \times 10^{8} \mathrm{~K}\right)$, these elements will fuel the first generation of fusion reactors. The fue? pellet performs most efficiently when the required temperature rise is achieved atiabatically with the compression necessary to obtain a sufficiently high reaction rate. The question of how to accomplish this operation by fuel-pellet design and beam energy pulse shaping is not addressed in this report. Neither do we discuss problems associated with the generation of pulses sufficiently energetic and intense to induce pelifet ignition. We assume that a compatible combination of pellet and 
driver (laser, light ion, or heavy ion beam) has been determined and use the above geireral characterization of the ICF process to provide a background for discussing ICF reactor system requirements.

Practical use of the ICF process requires a device--an ICF reactor--that satisfies the requirements listed $i_{4}$ the remainder of this section. The physical elements necessary to satisfy these requirements are listed below.

ICF reactors are required to induce nuclear fusion and to convert released energy into convenientiy usable forms, such as electricity and heat. The reactor system must satisfy a set of requirements that may be divided into two classes: (1) those pertaining to confinement and conversion of the released energy, and (2) those pertaining to repetitive fuel-pellet injection and ignition for long-term cyclic operation.

The first class comprises containment of fuel pellet microexplosions, conversion of neutron and plasma energies, and breeding of tritium to maintain self-sufficiency of fusion energy generation.

The second class comprises restoration of cavity conditions to the pre-explosion (ambient) state, injection of fuel pellets, and transport and delivery of a driver energy pulse to induce pellet ignition.

These requirements are satisfied, in general, by special-purpose subsystems of the ICF reactor. The subsystems must be conceived, designed, and matched properly to ensure reliable and economical reactor operation.

\section{B. Reactor System Components}

The core of the reactor is the microexplosion containment vessel. It consists of structural shells, a scheme to protect the innermost ("first") wall, and a blanket where neutron energy is converted into heat and tritium is produced (bred).

Structural shells usually consist of parts of spheres, cylinders, and cones. The exact shape depends on the first-wall protection scheme and on the detaits of the blanket design. The materials for the structural shells are selected according to heat transfer and heat resistance characteristics, neutron damage and activation characteristics, and structural strength.
Inertially confined fusion of a fuel pellet leads to the inciderice of the following forms of energy onto the first wall of the containment vesse 1: (1) electromagnetic radiation in which the dominant components are $x$-ray energy released after the reaction and laser light not absorbed by the pellet, (2) kinetic energy of the plasma debris, and (3) kinetic energy of fusion neutrons. Depending on the design concept, any of these forms can determine the lifetime of the first wall. Protecting this wall from the energy forms released during pellet microexplosions and modified by the cavity phenomena is the most challenging reactor design task.

Several schemes have been proposed to protect the first wall from radiation and ion damage. These schemes may be grouped into two generic classes: (1) cavities with permanent solid walls; the lifetimes of these walls can be extended by protective sacrificial liners and/or magnetic fields; and (2) cavities with renewable fluid walls, such as thin adhering liquid films or thick free-falling curtains.

A cavity wall protected by a buffer gas may belong to either class. If the gas is replaced after each microexplosion, it can be viewed as a renewable wall. If the gas is replaced only partially, the wall can be treated as solid, with the state of the gas defining the ambient cavity conditions. This example illustrates that the division between cavity phenomena and first-wal? problems is artificial and arbitrary.

Because tritium must be bred to ensure the self-sufficiency of fusion energy production, large amounts of lithium must be used in ail blanket designs. Fortunately, lithium has one of the largest known heat capacities and is liquid above $455 \mathrm{k}$. Because of these properties, the lithium used in most branket designs also is used as the heat transfer medium. For some special applications, for example, generation of very high temperature ( $2000 \mathrm{~K}$ ) process heat, the blanket must contain regions of (for example) graphite.

Because liquid lithium is chemically active, special attention must be given to the compatibility of materials. This disadvantage may be circumvented by designs that employ solid lithium compounds encapsulated in pellets ranging from 
approximately $1 \mathrm{~mm}$ to $1 \mathrm{~cm}$. However, efficient conveyance of these pellets may require development of special mechanical devices.

The kinetic energy of neutrons emitted during fusion of fuel atoms is converted in the blanket into heat that must be transported to heat exchangers and transferred to a working fluid, for example, water and steam, more suitable than lithium. Such a primary heat transfer loop alleviates probleins associated with the expected lithium contamination and permits utilization of we11-developed standard steam turbines. In addition to neutron energy, the energy content of the pellet debris and of the $x$ rays must be captured and used to ensure maximum efficiency.

The most efficient heat transport mechanism is forced circulation. Therefore, an ICF reactor system requires pipes, pumps, and other flowrelated equipment, in addition to heat exchangers and steam generators. These components can be modifications of analogous elements developed for the liquid metal fast breeder reactor (LMFBR) program and for commercial power plants.

ICF reactor designs need not be limited to the standard steam cycle, but may exploit other concepts for energy extraction and conversion, such as magnetohydrodynamic (MHD) generators or ion decelerators and high-temperature process heat generators that use radiative energy transfer. These devices can be described best in terms of specific reactor systems.

The unburned tritium in the cavity clearing passages, the tritium bred in the blanket, and the tritium that permeates into any cooling loops must be separated, processed, and delivered to the fuel-pellet manufacturing facility. Separation processes proposed include permeation through senipermeable tritium windows (large membranous surfaces), distillation of liquid lithium, coldtrapping in liquid metal loops, water-trapping or sparging in inert gas coolant loops, gettering, chemical binding (in molten salts), and the use of carrier fluids as in heat pipes. Technical and economic feasibility studies indicate that, at present, heat-pipe concentrators, gettering, molten salt extraction, and inert gas sparging are not competitive with the other methods. However, additional studies are needed for a more thoraugh evaluation.
Tritium recovered from tritium-breeding blankets and from coolant loops will contain large amounts of protium, and tritium recovered from cavity exhaust streams will be mixed with an approximately equal amount of unburned deuterium. Therefore, purification and isotopic separation are needed before processing in the fuel-pellet factory. Many processes can accomplish this task, but cryogenic distillation appears to offer efficiency, low cost, simplicity, reliability, and safely that will be difficult to improve.

The tritium throughput in an ICF reactor will be small by industrial standards, a few kilograms per day. The scale of the extraction, purification, and isotopic separation processes will resemble laboratory operations that are reasonably well understood. Therefore, developments are required mostly in the adaptation of current techniques to meet the long lifetime, reliability, and safety standards expected of a commercial operation.

Tritium containment is the primary concern in the selection of processes and in the design of tritium-handling equipment to protect the gublic, the operating and maintenance personnel, and the environment from the hazards of radioactivity.

Restoration of cavity conditions to the preexplosion ambient state requires removal of mass and heat between successive pellet burns. Mass removal associated with interpulse cavity clearing is accomplished with nozzles and vacuum pumps. Heat is transported by the primary loop to the heat exchanger.

Next to pellet ignition and energy containment and conversion, the most critical step required for demonstration of engineering feasibility is the development of a fuel-pellet delivery system that would operate in a commercial power plant continucusly with the necessary accuracy and reliability. Development of such a system requires solutions of the following technical problems:

- Pellet acceleration to the desired velocity;

- Pellet tracking; that is, acquiring and processing pellet position and velocity data to determine trajectory control requirements; and 
- Peliet and driver beam synchronization; that is, achieving coincidence of pellet and beam focal regions in space and time.

The last problem may be solved with simultaneous pellet guidance and beam pointing. Tradeoff studies are needed to determine the relative effectiveness and merits of these two functions.

The energy pulses generated in the driver are delivered into the reactor cavity where they ignite fuel pellets through a beam transport system composed of the following elements:

- Tubes, evacuated to a pressure sufficiently low to avoid beam quality degradation;

- Windows to contain pressure at beam tube entrances (for example, the pressure ratio between the laser amplifier and the beam transport tube may be as high as 1 000); they may be material or aerodynamic;

- Mirrors, both plane and focusing, with and without coolant passages; and

- Control systems for beam pointing, beam switching, and beam quality monitoring and correcting.

Specific requirements are given for laser driven systems. Analogous requirements, differing in detail, will be necessary for ion-beam driven systems.

The beam pointing system compensates for deviations in fuel-pellet trajectories by tilting the axis of each beam and translating its focus. Beam pointing in ICF reactors may be accomplished either with movable and deformable optical elements or with phase conjugation based on nonlinear effects. The technology base for the first option is large; it includes hydraulic and piezoelectric actuators. (The characteristics of electromechanical actuators appear to be outside parameter ranges of interest in laser reactors.)

Phase conjugation is a recent innovation and requires additional studies before its applicability to precise beam pointing can be evaluated adequately.

The beam-switching system is required for reactor designs in which several vessels are served by one driver. This function is accomplished with either tilting or rotating mirrors.
The task of the beam quality monitoring and correcting system is to sample the high-energy laser pulses incident onto the fue 1 pellets and to statistically correct high-order wavefront aberrations on the basis of this acquired information. The system is composed of a diagnostic sensor, a feedback loop, and a deformable mirror driven by piezoelectric actuators.

The technology to design and build an adequate beam transport system for ICF reactors appears to be available. Developments are needed to extend the sizes of individual elements and to achieve reliable and reproducible accuracy in repetitive (10- to $40-\mathrm{Hz})$ operations.

\section{REACTOR CAVITY PHENOMENA}

A. General Considerations

The particles remaining in ICF reactor cavities after each pellet microexplosion may interfere with subsequent laser beam propagation and/or fuel-pellet injection. This possibility imposes the only restriction on the interpulse state of the cavity medium. Therefore, a relatively wide choice of ambient conditions may be exploited to enhance protection of the innermost (first) cavity wail and to increase energy extraction. Consequently, a variety of physical phenomena may develop that dominate the ICF reactor cavity design, depending not only on cavity conditions between pulses but also on detajls of fuelpellet structure. These phenomena modify the forms of energy released in the pellet microexplosion and lead to different cavity design problems. Therefore all relevant cavity phenomena must be analyzed thoroughly before first-wall design problems can be identified and solved.

Interpulse ambient cavity conditions nay be grouped into three broad categories.

- Vacuum, when the mean free path of pellet debris ions is longer than or comparable to a characteristic cavity dimension for both momentum and charge exchange reactions;

- Gaseous atmosphere (possibly ionized by the bisst of prompt radiation emitted from the pellet), in which the debris ior mean free paths are short for at least one kind of encounter; and 
- Mixture of vapor and liquid phases.

of course, convenient and economic operating conditions may fall into some regime between these categories. In such cases, satisfactory modeling and analysis of cavity phenomena may be quite difficult.

First-wall protection schemes for ICF reactor cavities were characterized briefly in Sec. I. clearly, not all protection schemes and interpulse ambient conditions are compatible. The reasons may be physical and/or economical. Because a high vacuum is difficult if not impossible to maintain in any but solid wall cavities, a vacuum can be used only with cavity concepts that employ bare refractory metal walls, sacrificial liners, or magnetic field protection. Vapor and liquid phases will be present in the cavity when liquid metal (for example, lithium) is used for wall protection either as an adhering thin film or as free-falling jets or curtains.

Depending on the choice of ambient cavity conditions and on the fuel-pellet structure, the following phenomena may dominate cavity design:

- Unimpeded energy propagation from the exploded pellet to the cavity wall in the forms of neutrons, $x$ rays, and debris ions;

- Debris ion expansion in the presence of a magnetic field through vacuum or through a background medium in which an average ion mean free path is short relative to a representative cavity dimension for at least one type of encounter;

- Large-scale (blast and/or acoustic wave) and small-scale (turbulence) motions of the continuum buffer medium induced by pellet explosion and expansion; and

- Hydrodynamic motion with evaporation and condensation effects.

The effects of radiant energy transport may or may not be negligible in the last three classes of cavity phenomena.

Modeling and analysis of some of these phenomena are discussed below without reference to a particular cavity concept.

\section{B. Cavity Phenomena Modeling}

The objectives of modeling and investigating cavity phenomena are to determine

- transient thermal and mecnanical wall loadings;

- potential damage mechanisins to the cavity first wa11; and

- requirements for the restoracion of anbient cavity conditions between successive fuelpellet microexplosions for repetitive operation.

1. Ion Expansion Model. The expansion of pellet microexplosion products through a vacuum does not require special analysis blit leads directly to investigations of the interaction of these products with the first-wall material. However, establishing and maincaining a hard vacuum between pellet microexplosions may require more equipment and time than is practicable. Therefore, it is desirable to model the expansion of debris ions through as dense an atmosphere as can be tolerated. Such an atmosphere also may alleviate some first-wall design problems. For wider applicability (for example, to the magneticaliy protected reactor cavity concept), the effects of magnetic field are included in the mode' described below.

The requirement for the transmissibitity of laser beams imposes an upper limit on the admissible gas density $\mathbf{n}$ in the reactor cavity. At present, this limit is not known precisely, ${ }^{1}$ but it is not expected to exceed a value between $10^{75}$ and $10^{16}$ atoms $/ \mathrm{cm}^{3}$. A representative volume of an ICF reactor cavity (4-m-long cylinder with a 2-m radius) is $5 \times 10^{7} \mathrm{~cm}^{3}$. The total number of atoms in such a cavity may be between $5 \times 10^{22}$ and $5 \times 10^{23}$. If only $0.1 \%$ of the nominal pellet yield of $150 \mathrm{MJ}$ is absorbed by these atoms, the energy per atom wiit range between 19.0 and $1.9 \mathrm{eV}$, sufficient to jonize and thus to transform the nedium into a perfectly conducting plasma.

At the above conditions, the collisional cross sections $\sigma$ for the cavity medium particles are approximately $10^{-15} \mathrm{~cm}^{2}$ (Refs. 2,3); consequently, the mean free path $\lambda(\lambda \approx 1 / \pi n o)$ is 
between 0.30 and $0.03 \mathrm{~cm}$ long. These estimates show that phenomena with characteristic lengths of $1 \mathrm{~m}$ or more can be modeled with a continuum description of the background medium. However, the pellet debris ions generated in the microexplosion behave differently. For example, calculations based on the Lindhard stopping theory show that at the neon density of 1.5 $\times 10^{16}$ atoms $/ \mathrm{cm}^{3}$ (the upper ailowable 1imit) the mean debris ion range is between 2 and $5 \mathrm{~m} .{ }^{4}$ Ion ranges of that magnitude are comparable to or larger than radii of ICF reactor cavities and much larger than the mean free paths in the background medium. Therefore, the ion motion cannot be approximated within the framework of a fluid model but must be determined from kinetic theory. In our analysis this is accomplished by calculating trajectories for a statistical sample ( 5000 to 20000 ) of ions.

Consistent with previous estimates, the plasma model for the analysis of cavity phenomena contains three parts: background fluid, a collection of discrete, relatively high-energy ions, and an electromagnetic field. The single component background fiuid is initially ionized by the flash of $x$ rays emitted from the burning $D-T$ fuel pellet to a degree that makes it a perfectiy conducting plasma. Therefore, its behavior is described by modified Lundquist equations supplemented with an appropriate equation of state and a rate equation that determines changes in the degree of ionization. The motion of pellet debris ions is described with a representative sample of ion trajectories calculated by integrating the equation of motion for each simulation ion with the Lorentz force contributing to the acceleration. The model also includes phenomenological terms that describe the momentum and energy transfer between fluid and discrete ions and a rate equation that determines the average ion charge.

The electromagnetic field is described with Maxwell's equations in which the displacement current is neglected. Ohm's law is replaced with postulates that the electric field is always and everywhere perpendicular to the magnetic field, that local-charge neutrality prevails, and that the electrons are massless and drift without inertia in the direction perpendicular to electric and magnetic fields. Thus, the model constitutes a significant generalization and extension of considerations introduced by Dickman, Morse, and Nielson ${ }^{5}$ and developed further by Goldstein and coworkers. 6

To be specific, we present the differential equations and the constitutive relations corresponding to the ajove plasma model. For conciseness, we employ vector representation and write the differential equations in conservation form to facilitate future discussion of finite difference approximations. In our notation convention, the operator acts on all factors that follow it.

The equations expressing conservation of mass, momentum, and energy are

$$
\begin{gathered}
\frac{\partial o}{\partial t}+\nabla \cdot(o u)=0, \\
\frac{\partial}{\partial t}(o u)+(\nabla \cdot u)(o u)+\nabla p \\
+\frac{1}{C} B \times\left(J-J_{I}\right)+\Gamma_{B}=0,
\end{gathered}
$$

and

$$
\begin{gathered}
\frac{\partial}{\partial t}\left[o\left(\frac{\partial}{2}|u|^{2}+\varepsilon\right)\right]+(\nabla \cdot u)\left[o\left(\frac{1}{2}|u|^{2}+E\right)+p\right] \\
=E \cdot\left(J-J_{I}\right)+S_{B},
\end{gathered}
$$

and the equation expressing the change in the ionization fraction $\alpha$ is

$$
\frac{d \alpha}{d t}=F_{1}(0, T)
$$

In the above equations, $F_{1}$ is a given function, $t$ denotes time, 0 is the mass density of the background fluid, $u$ is the velocity vector, $p$ is the pressure, $c$ is the speed of light in a vacuum, $B$ is the magnetic field vector, $J$ is the total current vector, $J_{I}$ is the current carried by the debris ions, $E$ is the internal energy, $E$ is the electric field yector, and $T$ is the temperature. The symbol $\nabla$ denotes the gradient operator and $|u|$ is the magnitude of the vector $u$. The terms $\Gamma_{B}$ and $S_{B}$ represent, respectively, the collisional momentum and the energy interchange between background fluid and debris ions.

The trajectories of the debris ions are determined $^{5,6}$ by integrating the equations of motion 


$$
m_{i} \frac{d v_{i}}{d t}=q_{i}\left(E-\frac{1}{c} B \times v_{i}\right)-r_{i},
$$

where $m_{i}, v_{i}$, and $q_{j}$ are the mass, velocity vector, and charge of the $i^{\text {th }}$ ion, and $\Gamma_{i}$ is the collisional drag force exerted by the background fluid on the ion. In practice, Eq. (5) is integrated for simulation particles representing many ions of a given element; a typical calculation may contain any number (between 5000 and $25000)$ of simulation particles. The charge of the simulation particle will change according to

$$
\frac{d q_{i}}{d t}=F_{2}\left(0, T, m_{i}\right)
$$

where $F_{2}$ is the recombination rate for each ion material.

The electromagnetic field is governed by Maxwell's equations, which, with the previously indicated approximations, reduce to

$$
\frac{\partial B}{\partial t}=-C \nabla \times E
$$

and

$$
J=\frac{c}{4 \pi} \nabla \times B .
$$

Equations (1)-(8) do not form a closed system and must be supplemented with auxiliary relations among different physical quantities. These are of two kinds: those relating the thermodynamic variables $T, p, \varepsilon$, and $\circ$ (known as equations of state) and those relating the current density $\mathrm{J}$ and the electric field E (known as Ohm's law).

The thermodynamic variables are related with

$$
T=\frac{m(\gamma-1)}{\left(N_{A} O+n m\right) k} D E
$$

and

$$
p=\left(\frac{N_{A^{0}}}{m}+n\right) k T,
$$

where $m$ is the molar mass of the background fluid, $\gamma$ is the ratio of specific heats lassumed constant), $N_{A}$ is Avogadro's number, $k$ is the Boltzmann constant, and $n$ is the electron density.

Consistent with the previously stated hypothesis about the behavior of the electrons, the expression for the current density $\mathrm{J}$ is

$$
J=J_{I}+\frac{N_{A} e}{\sqrt{\pi}} \text { Zoou }- \text { nec } \frac{E \times B}{\mid B I^{2}} \text {. }
$$

where $Z$ is the atomic number of the background fluid, $e$ is the charge of an electron, and the debris ion current density $J_{I}$ is given by

$$
J_{I}=\sum_{V O L T M E}^{\text {UNIT }} q_{j} v_{j} .
$$

From the assumption of charge neutrality, the electron density $n$ is

$$
n=\frac{1}{e} \sum_{\text {VOLUNE }}^{\text {UNIT }} a_{i}+\frac{N_{A^{O}}}{m} Z_{\alpha} .
$$

The relation between current density and electric field (Ohm's law) may be solved for the electric field $E$. Use of the postulated orthogonality of the electric and magnetic fields makes the expression

$$
E=\frac{B}{n e c} \times\left(J_{I}+\frac{N_{A} e}{m} \text { Zaou }-J\right) .
$$

Equations (1)-(14) constitute a closed seifconsistent system describing the plasma model.

The expression for the drag force $i_{i}$ is derived easily fren the concept of the stopping power SP. If $x$ is the distance along the ion path and KE is its (kine iic) energy,

$$
\frac{d(K E)}{d(O x)}=S P
$$

But $\quad K E=1 / 2 m v_{i}^{2}, \quad d(o x)=\alpha d x \quad$ ifor locally constant $o)$, and $v_{i}=d x / d t$; hence

$$
\frac{d(X E)}{d(D x)}+\frac{m v_{i}}{D} \frac{d v_{i}}{d x}+\frac{m}{D} \frac{d v_{i}}{d t} .
$$

Because $d v_{j} / d t$ is the acceleration, by Newton's law, $m\left(d v_{j} / d t\right)=o(d K E / d o x)$ is the force $r_{i}$. Using Eq. (IE), we obtarn $T_{i}$ $=$ oSP. For numerical calculations, $S P$ is expressed as the sum of eleitronic, $S_{P_{e}}$, and nucleonic, $\mathrm{SP}_{n}$, contributions; that is,

$$
\Gamma_{i}=o\left(S P_{e}+S P_{n}\right)
$$


The stopping powers of different media have been investigated extensively both theoretically and experimentally. Various physical mechanisms may doninate or contribute to this phenomenon, depending on the range of parameters in which the calculations are performed. Examples of mechanisms relevant to ICF reactor studies are excitation and ionization of the background fiuid (Bethe-B)och processes), Coulomb interactions between debris and background ions, and Couiomb interactions between debris ions and free electrons. The proper expressions for Eq. (16) depend on the conditions prevailing in the cavity and on the desired degree of approximation.

Consistent with the sign convention in Eqs. (2) and (5), the reaction force on the background medium $\Gamma_{B}$ is the sum of $\Gamma_{i}$ over all ions present in each unit volume. In all calculations of the forces and of the ion energy loss $S_{B}$, the relative velocity between the ions and the background medium is lised.

The characteristic time for the ionization/recombination procsisis governed by $\mathrm{Eq}$. (4) may be very short compared to the integration time step determined by the fiuid dynamic phenomena. In that case, instead of Eqs. (4) and (9), use relations based on the Saha equation. These can be of two types: numerically calculated and tabulated compatible values of ionization fraction $\alpha$, internal energy $E$, and temperature $T$ (Ref. 7 ) or anaiytic approximations. An example of the letter applicable to the study of ICF reactor cavity phenomena is the coronal model of Shearer and Barnes, 8

$$
\alpha=\frac{26}{Z}\left[\frac{k T(k e V)}{1+(26 / Z)^{2} k T(k e V)}\right]^{1 / 2},
$$

that satisfactorily approximates experimental results in parameter ranges of our interest.

2. Ion Expansion, Computational Procedure. Determining the time evolution of conditions inside ICF reactor cavities following a fuelpellet microexplosion requires solving an initial value problem for the above equations inside a finite domain bounded by rigid electrically conducting walls. Therefore, the boundary conditions are vanishing of the tangential component of the electric field and of the normal component of mass velocity and continuity of the tangential component of the magnetic field. Because the events following pellet microexplosion occur in times that are short in comparison to the characteristic time for the magnetic diffusivity in the wa'l material, it is appropriate also to require that the magnetic field at the boundary remain unchanged.

The plasma model used in our analysis of the cavity phenomena does not contain viscosity and heat conductivity; therefore, the above stated boundary conditions are the only requirements imposed by the physical considerations. However, to make the initial-boundary value problem numerically well posed, the boundary conditions must be specified for ali primary variables (that is, those whose time rate of change is given by differential equations). To obtain these additional conditions, we anticipate that the cavity phenomena will consist of wave reflections and postulate that the reflections will be predominantly normal. Consequentiy, the reflection conditions (that is, yanishing of the normal derivative at the boundary) should be approximately valid locâlly for pressure, density, and the tangential component of the velocity. We also postulate that the ions will adhere to the wall upon striking it. This is achieved by removing the ion from the comratationai scheme whenever its trajectory intersects the boundary. A record of the time, position, and ion energy at impact is retained for use in analyses of first-wall effects described in the next section.

Solutions describing the evolution of plasma conditions inside reactor cavities are obtained by numerical integration of the governing equations. Differential Eqs. (1)-(4) and (7) are replaced with finite difference approximations using the Lax-Friedrichs scheme introduced by $\operatorname{Lax}^{9}$ and applied effectively by Bohachersky and collaborators $^{10-12}$ to calculate multidimensional flows with shock waves and radiation effects. Equation (5) is integrated straightfonwardy in time, and the auxiliary variables are evaluated at each time step and smoothed with a four-point averaging process to ensure a stable and accurate computation. 
Exploratory calculations and supporting analysis of debris ion expansion through a background fluid showed that the relaxation time for the transfer of momentum and energy from the ions to the fluid is much shorter than the characteristic fluid-dynamic time because, in the early stages of the expansion, the mass ratio of the ions to the entrained fluid is much larger than unity. Therefore, the complete set of governing difference equations is stiff. The degree of stiffness may be appreciated from the fact that at the characteristic interaction time of approximately $10^{-12} \mathrm{~s}$, the typical characteristic fluid-dynamic time is only approximately $10^{-6} \mathrm{~s}$. The resolution of the associated computational difficulties consisted of finding an approximate but asymptotically correct characterization of the ion-fluid interaction in each computational cell and incorporating the corresponding expressions for the veincity and internal energy into the code.

The integration is initialized by (1) determination of the desired magnetic field distribution (either by placing coils at appropriate locations or by solving the boundary value problems for the magnetic vector poteniial), (2) placement of simulation particles into desired locations with desired velocities (energie;), and (3) prescription of initial distributions for the density, velocity, and temperature of the background fivid.

After initialization, the variables are advanced in discrete time steps in the following order.

(1) The simulation particles are moved in accordance with Eq. (5).

(2) The values of density, velocity, and internal energy of the fluid are advanced by using Eqs. (1) $-(3)$.

(3) The auxiliary thermodynamic quantities lincluding ionization fraction, a) are determined.

(4) The current is calculated with Eq. (8).

(5) The electric field is calculated with Eq. (14).

(6) The magnetic field is advanced with Eq. (7) completing the cycle.
The computations are continued until al1 simulation particles disappear into containient walls or until a quasi-steady state is reached.

3. Ion Expansion, Illustrative Results. Results of the above described plasm modeling are shown in Figs. 1-8 to illustrate qualitatively the general behavior of the plasma.

Figures 1-3 illustrate ion expansion in a vacuum, that is, with the background fluid omitted. In this case, of course, the computations are simplifier significantly; in particular, it is possible to combine the computations of electric and magnetic fields into the solution of a single Poisson's equation at each time step. The physical assumptions leading to this approximation have been formulated and discussed in Ref. 5, and the first studies of ion motion in ICF reactor cavities were reported in Ref. 6 .

Figure 1 illustrates successive stages of fuel-pellet debris expansion in a cylindrical reactor vessel that opens into a large conical end surface, called an energy sink. Wich absorbs the ion energy with acceptable surface loating. The externally generated axial magnetic field of approximately $1000 \mathrm{G}$ protects the cylinder wall by deflecting the ions and guiding them into the energy sink. Figure 2 is a cumulative histogran of energy and particle depositions on the confining walls that shows the magnetic field functioning as intended. Because the values plotted in Fig. 2 must be divided by the element of the cone area at each axial location to obtain deposjtion per unit area, the surface load intensity increases as the cone vertex is approached and the ion strean is collimated along the reactor vessel axis. This suggests that an cpening should be provided at the axis of symetry through wich the ions can escape. Their kinetic energy can be converted into useful form outside the vessel with a direct energy conversion device such as an mo generator. If that option is exploized, the conical end of the cylind;ical vessel need not be enlarged. The shapes of the expanding debris cluster and of the magnetic contour 3 ines in such a cavity are shown in Fig. 3a, and the intensity of the fields is shown in Fig. 3b. Formation of the "magnetic funnel" that protects the miterial wall and directs the debris ions into the inlets to MWD channels is significant. 
IIME $\quad 6000806$

RMax 9 950t $\cdot 07$

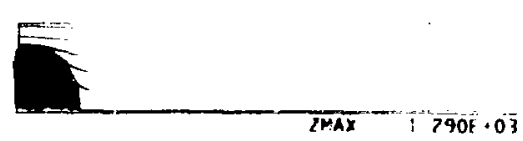

IIMt 18001 OS
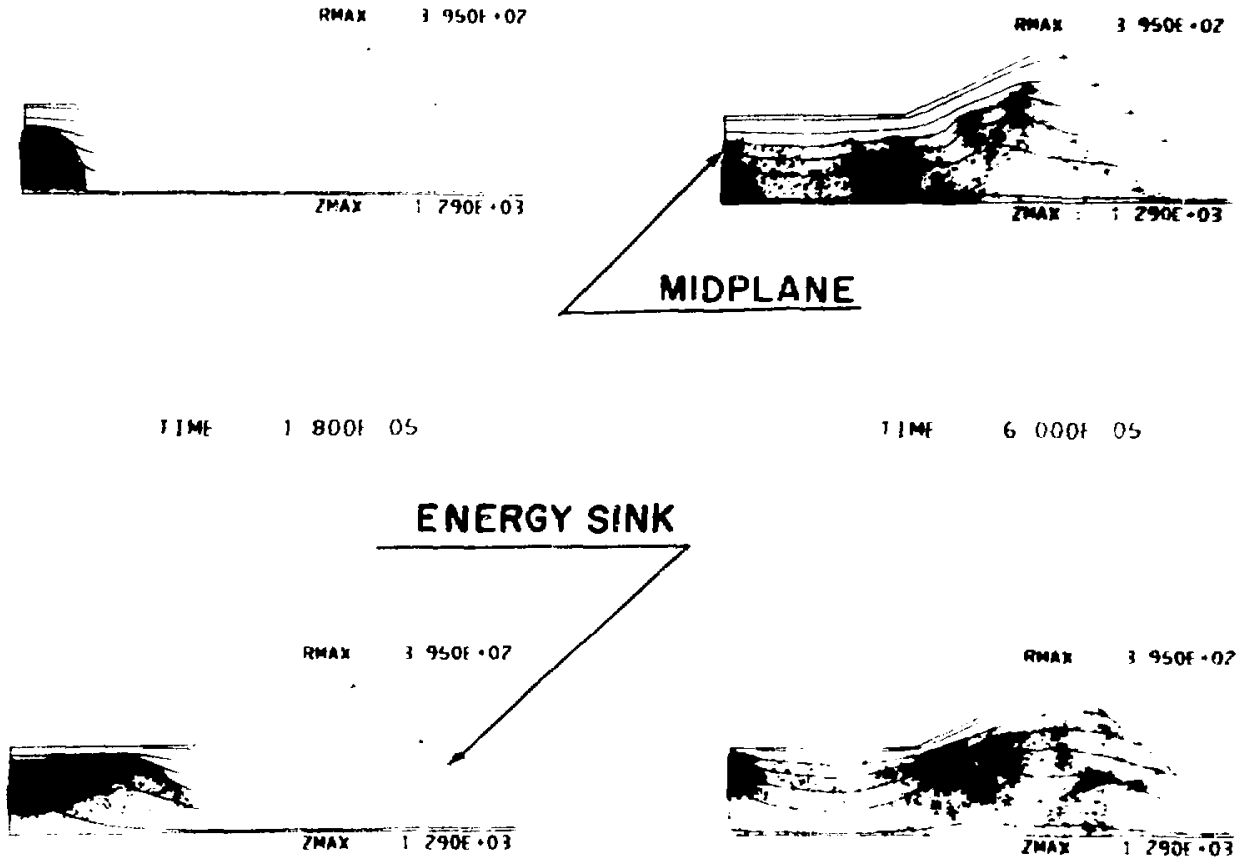

IIMF 3 600t OS

11 Ime 78005 OS
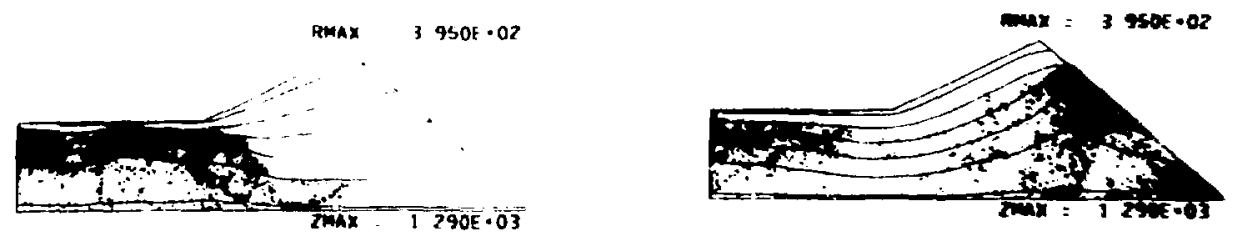

Fig. 1. Ion expansion in vacuum. 


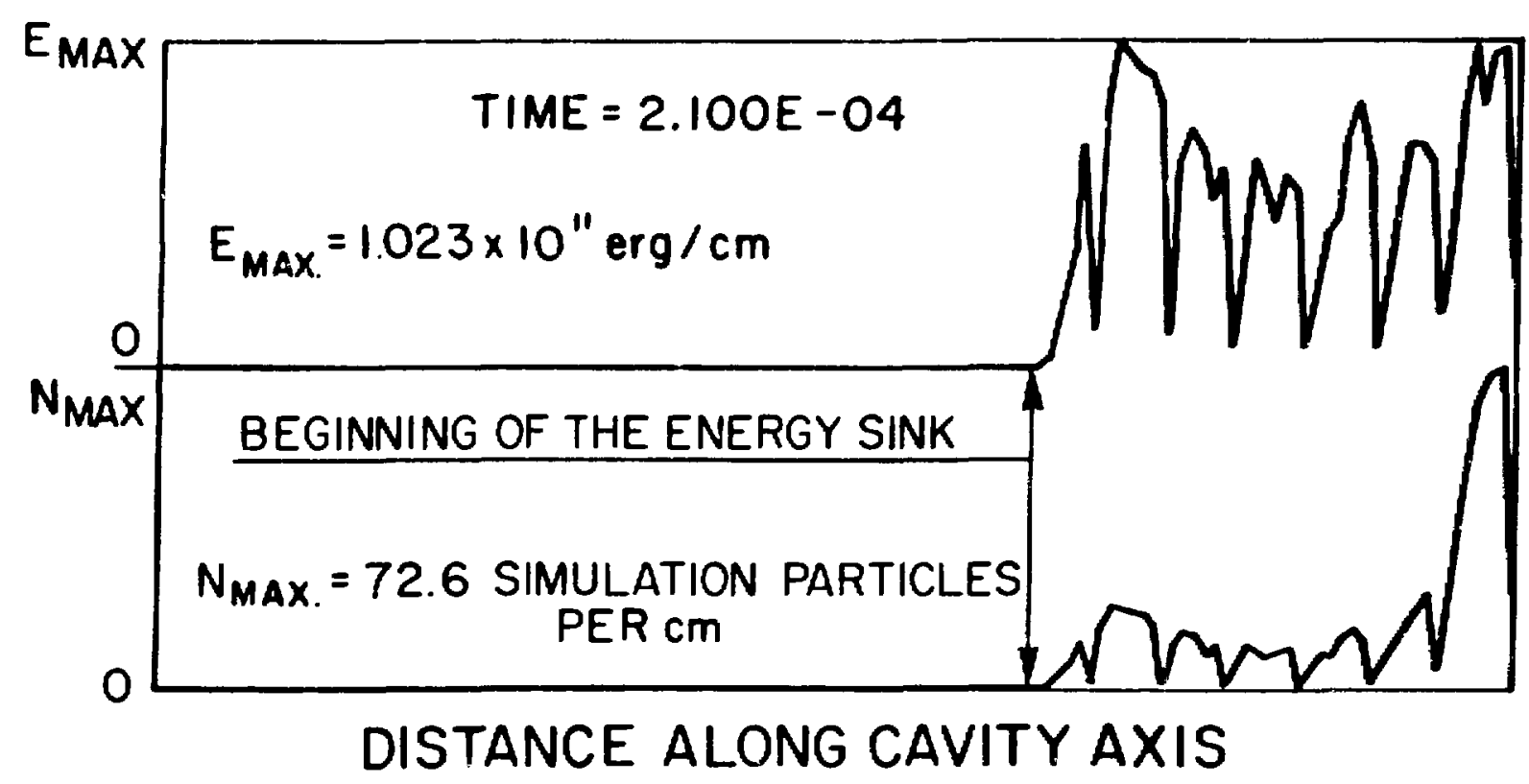

Fig. 2. Ion eneray deposition in the energr sink.

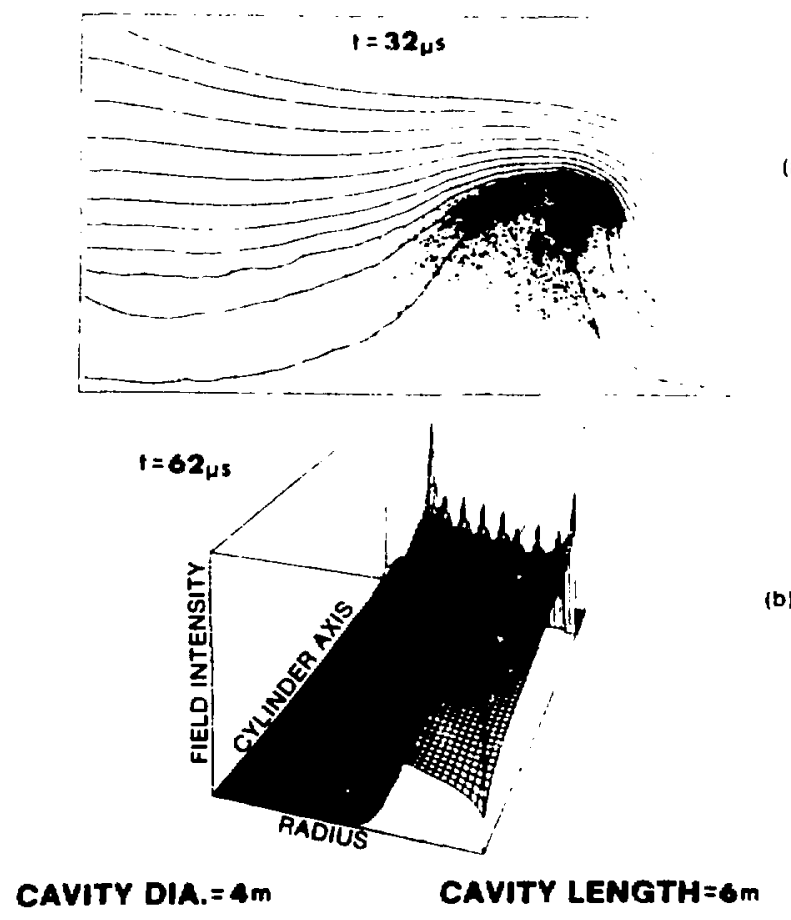

Fig. 3. (a) Debris expansion in a 6-kG field. (b) Magnetic field intensity. (a)

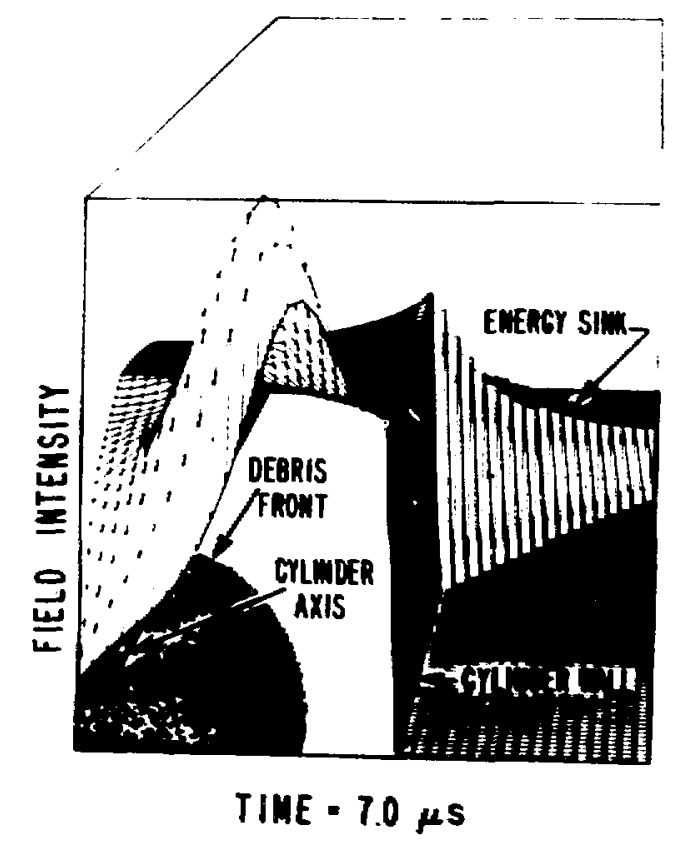

fiq. 4. Ion expansion and magnetic field displacement. 


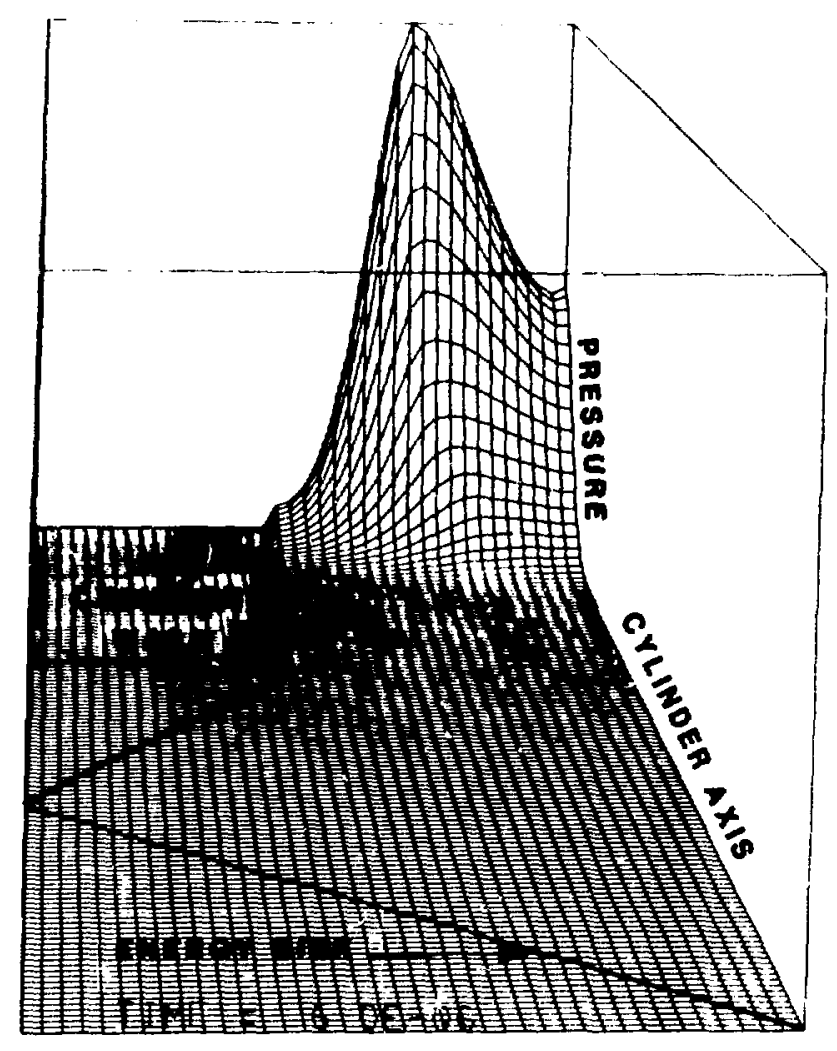

TIME $6.0 \mu \mathrm{s}$

Fig. 5. Pressure wave formation.

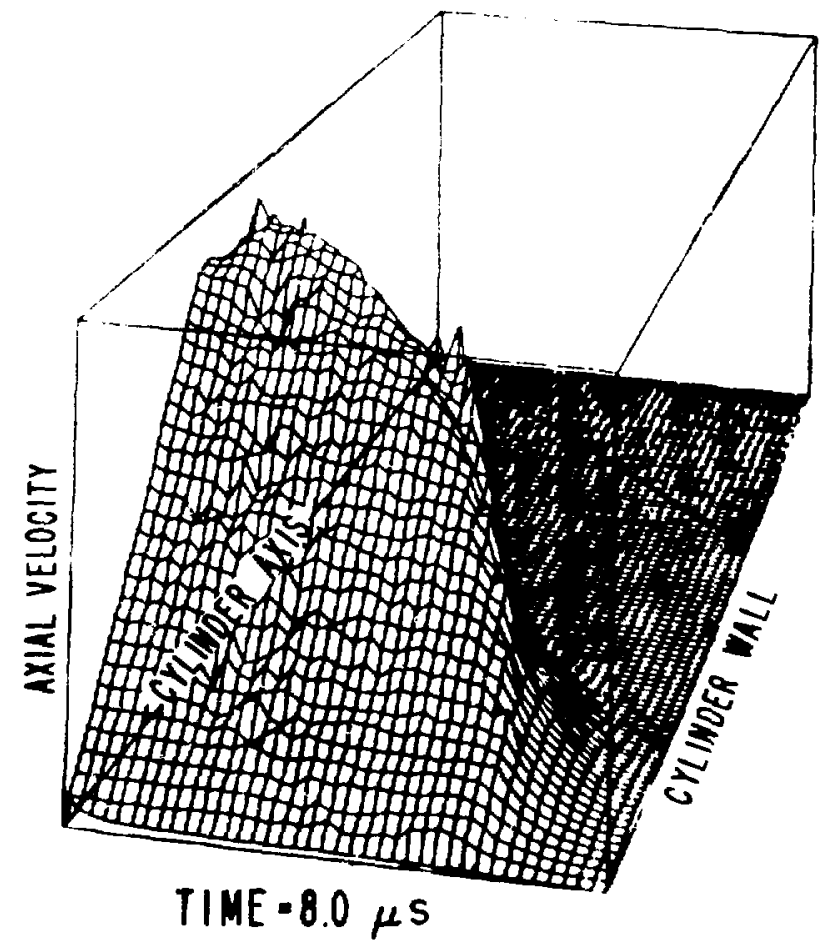

Fig. 7. Axial velocity distribution in reactor cavity.

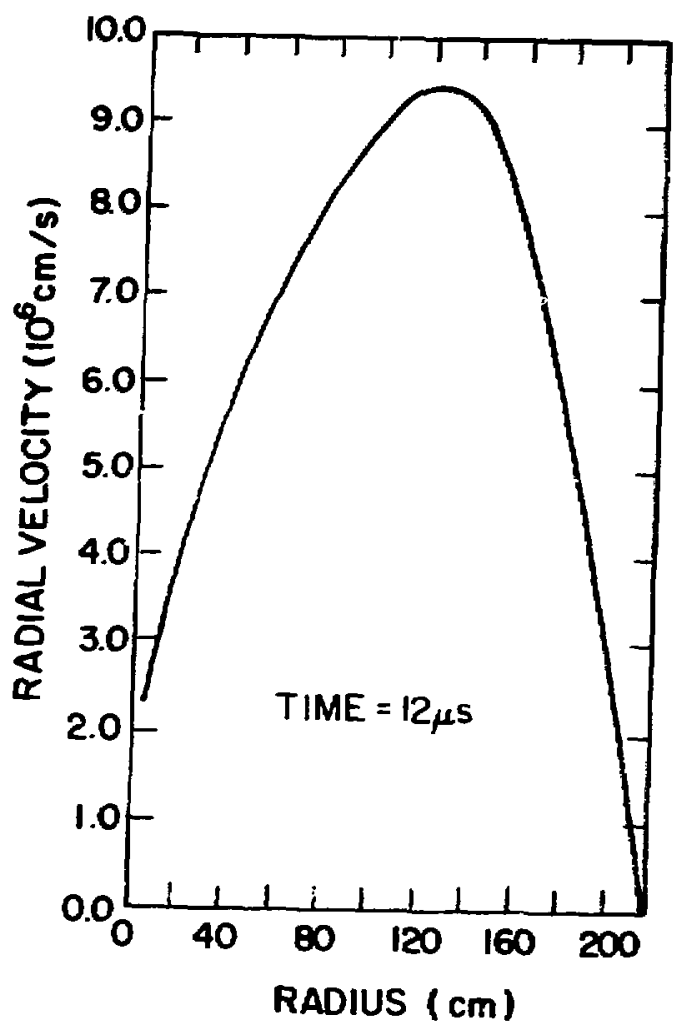

Fin. 6. Radial velocity distribution.

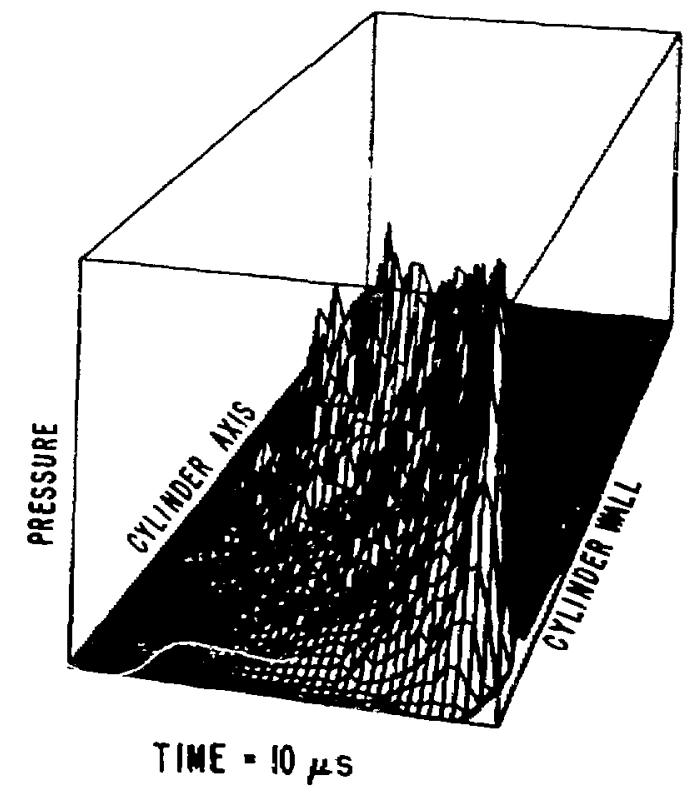

Fin. 8, Pressure distribution in reactor cavitv. 
Figures 4-8 illustrate the microexplosioninduced expansion of debris ions through a background fluid with an imbedded magnetic field. Figure 4 shows the correlation between the expanding debris front and the location of the maximum magnetic field intensity, and Fig. 5 shows the pressure wave generated by the interaction of pellet products with the background fluid. Figure 6 shows the corresponding radial velocity profile of the background fluid at the reactor cavity midplane.

A typical axial velocity distribution is shown in Fig. 7; evidently the background fluid acquires a nearly uniform axial velocity well suited to driving MHD generators.

The solutions indicate that the infinitely conducting background medium attaches "inertia" to the magnetic lines of force and irhibits their compression. Except for this indirect influence, the background fluid does not affect the ion trajectories significantly before the pressure waves reflect from the cavity walls. However, the ions impart such a high velocity to the background fluid that despite large energy deposition, the temperature and density inside the region of expanding ions decrease to very low vaiues. Thus, the individual ions act analogousiy to a jet ejector, and the effect is quite strong. It reduces the density inside the expanding ions to a value less than one-thousandth of the initial ambient density. Figure 8 shows the pressure wave generated by the interaction of peliet products and background fluid and the low-temperature (because temperature is proportionai to pressurel region that develops behind it as a result of the fluid expansion.

This analysis of the effectiveness of the magnetic protection of the first warl of the containment vessel must be supplemented by estimates of the growth rates of flute instabilities. A) though these rates cannot be obtained within the framework of the present model, an independent analysis 13 showed that the relevant doubling times exceed the time required to clear the cavity of the debris; therefore, these phenomena will not compromise the concept.
4. Radiation Effects, Fireball and Blast Wave. Eneray transfer from the debris ions to the background fluid contained in the above ion expansion model is not the only mechanism capable of generating a pressure wave--radiation transport is another. In this section we discuss the effects of radiation transport to complement our analysis of ion-background fluid interactions.

Radiation energy transport influences cavity phenomena whenever the opacity of the background fluid induces absorption of enough energy to affect significantly the state of the fluid. Thus, accurate opacity tables and an accurate equation of state are essential for understanding the absorption of radiation energy and subsequent phenomena. These quantities are such camplex and sensitive functions of the material and of the temperature that any quantitative discussion of radiation effects is valid only for a particular fluid and within particular ranges of temperature, density, and energy release of the fuei pellet. Therefore, the following discussion is necessarily limited to qualitative aspects of possible pheromena and to commerits about the possibilities of their occurrence or nonoccurrence in specific circumstances.

The study of phenomena associated with very large energy releases in very small volumes was initiated by Bethe and his colleagues in Los Alamos ${ }^{14}$ and was extended and incorporated into a general treatment of high-temperature hydrodynamic phenomena by Zel'dovich and Raizer. ${ }^{15}$ The following discussion is based on the updated analysis of Bethe. 16

The D-T fusion reaction inside a fuel peliet gives rise to the following sequence of events. The liberated energy heats the pellet material and a sphere of surrounding fluid to a very high tenperature (millions of degrees). The exact extent and state of this region are not known because it is very difficult to detemine theoretically the transfer of energy from the pellet material to the surrounding fluid. Following the initial stage of energy deposition. the heated material radiates and expands until the temperature at its boundary decreases to a value at wich the cavity medium becomes opaque to the dominant radiative emission. Inside the radiating and expanding surface 
the material is transparent to its radiation; therefore, this region is isothermal. The density and temperature dependence of the absorption properties of the cavity fluid determines whether such a blocking layer develops at a radius smaller than the radius of the reactor cavity.

When the radiative energy loss from the heated mass is reduced to a diffusion-like process, the velocity of the radiative energy transport becomes much lower than the velocity of the shock wave. Therefore, the shock wave separates and propagates ahead of the luminous fireball. This shock may or may not be sufficiently strong to ionize the fluid, an action that depends on the properties of the medium and on the characteristics of the energy release.

Because of spherical divergence, the layer of shock-heated fluid expands adiabatically, and therefore its temperature decreases. This generates a "cooling wave"15,16 that propagates into the expanding fireball. The cooling wave is strong enough to redice the fireball temperature to a value at which its surface again becomes transparent to the radiating temperature of the fireball. From then on, energy is lost by volumetric radiation. Again, this may or may not take place before the shock wave reaches the cavity wa17.

In summary, the events outlined above lead to the development of up to five regions behind the

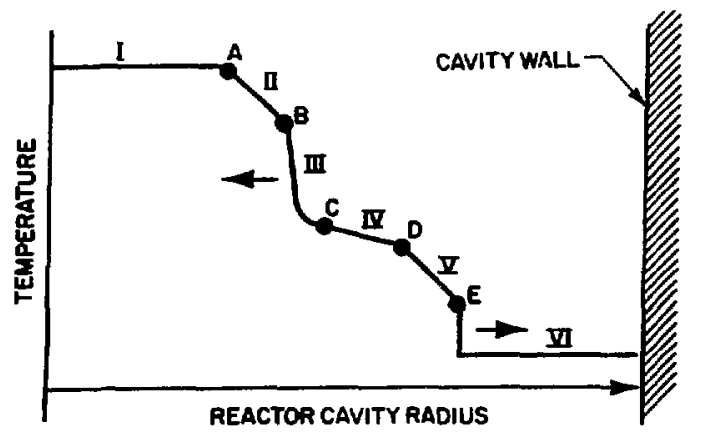

Fig. 9. Temperature distribution in reactor cavity (schematic). I - isothermal region. II adiabatic expansion region in front of the cooling wave. III - cooling wave. IV adiabatic expansion region behind the cooling wave. $V$ - adiabatic expansion region behind the shock. VI - interpulse ambient conditions.

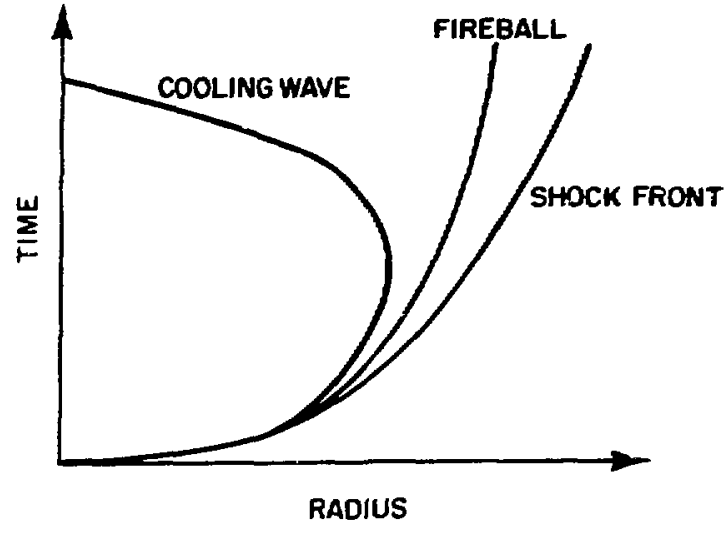

Fig. 10. Wave propagation in reactor cavity (scinematic).

shock. These regions are shown in Fig. 9, which is borrowed from Ref. 16. Figure 10 shows the propagation of the boundaries between these regions.

For a specific fluid and a specific energy release, we can estimate quantitatively the extent and properties of the regions depicted in Fig. 9. Unfortunately, the detailed estimates derived in Refs. 14-16 were obtained for energy releases of approximately $100 \mathrm{GJ}$ in air. Therefore, they are not applicable to reactor studies because even though the results may be scaled with the energy yield, the thermochemical and optical properties of the cavity fluids considered are entirely different from those of air.

For example, in calculating the above phenomena applicable to ICF (1ight ion driver) cavity conditions ( $30 \mathrm{mu}$ of energy deposited inside a $10-\mathrm{cm}$ radius in argon at a pressure of 50 torr and density of $\left.1.114 \times 10^{-4} \mathrm{~g} / \mathrm{cm}^{3}\right)$, Peterson and Moses ${ }^{17}$ found that most of the energy escapes as radiation, so the shock, in.:separates from the fireball at a radius of approximately $270 \mathrm{~cm}$, is very weak. Also, the cooling wave does not develop because the radiative loss and the expansion of the fireball are sufficient to reduce the radiating surface temperature below transparency, wich, for noble gases (argon), is high relative to molecular gases. Thus, for these ICF reactor cavity conditions, Regions III and IV of Fig. 9 do not develop. 
5. Similarity Solutions, waste Energy. When certain cavity conditions and energy release characteristics are present, the shock separates from the fireball at a radius that is small compared to the cavity size, and the shock is too weak to ionize the fluid. Under these iircumstances, shock propagation and characteristics can be determined from the Taylor-Sedov similarity solution. 14,i8 This solution is accurate provided tha energy generating the shock and the effective adiabatic exponent of the medium are determined correctly.

The Taylor-Sedov solution is theoretically valid for point energy releases; in practice it is a good approximation if the mass swept by the shock is large relative to the mass of the pellet. In ICF applications, this approximation will be valid only for electron or light ion drivers when the ambient cavity pressures are nearly $1 \mathrm{~atm}$. In general, the total mass of buffer gas in the cavity is expected to be only comparable to the pellet mass. A valid description of the blast wave in this general case has been obtained by Freiwald and Axford ${ }^{19}$ by modifying the classical Taylor-Sedov similarity solution to account for the non-negligible pellet mass. Although their analys is significantly improves the similarity solution when fluid and pellet masses are comparable, it requires numerical integrations, depends on ambient conditions, and, like the similarity solution, is sensitive to the effective adiabatic exponent of the medium. Therefore, their results must be evaluated for each specific case to obtain a quantitative description of the cavity phenomena. Details of the Freiwaid-Axford analys is and representative results are contained in Refs. 1 and 19 and will not be discussed further here.

of interest in the study of ICF reactor cavity phenomena is the concept of "waste energy" 14 introduced by 6. 1. Taylor. In investigations of blast effects, this energy is defined as the energy remaining in the hot fluid traversed by the shock after the pressure decays to the ambient value. Because this energy is no longer available to drive the shock, it is considered "waste." In the study of cavity phenomena, a more useful definition is the internal erergy of the fluid at the time when wave motion has dissipated and the medium has attained an approximately homogeneous state. With this definition, waste energy is the amount of heat that must ba removed between successive pulses to ensure long-term steady-state operation.

The waste energy for the Taylor-Sedov similarjty solution was calculated in Ref. 14 and found to increase with secreasing adiabatic exponent. The values of waste energy could reach a significant fraction (> 1/2) of the pellet $x-r a y$ and debris energy yield because the energy of the fusion reaction is released at a very high temperature, and most of it goes into heating the surrounding material. Therefore, when a gas buffer is used for first-wall protection, a substantial amount of heat must be removed from the cavity between pulses.

The waste energy (heat) can be removed either by radiation to the cavity wall or by convection (by circulation of tile cavity fluid). Removal of heat by radiation requires operation at temperatures that may be higher than practicable. For example, the temperature required for argon was determined by Peterson and Moses 17 to be approximately $8000 \mathrm{~K}$.

\section{c. Restoration of Cavity Environment}

After each pellet microexplosion, the cavity environment must be restored to its ambient preexplosion conditions to ensure

- injection of the fuel pellet with required consistency,

- propagation of laser beams with required quality and accuracy, and

- long-term operation by maintaining the energy and mass content of the cavity at a constant average level.

Each of these requirements involves removing a certain amount of mass and heat during every cycle of reactor operation. It is impossible to identify in advance the dominant (controliang) constraint without considering a specific design configuration and mode of operation. In this sec$t$ ion we derive genera? relations among different 
cavity parameters, which provide a lower bound for the time required to remove the necessary amount of mass from the cavity. Heat transfer requirements and blanket phenomena are analyzed in a later section.

1. Reservoir Exhaust. We postulate that the mass is exhausted from the cavity through a convergent-divergent (DeLaval) nozzle and that the pressure on the downstream side (at the entrance to the heat exchanger or condenser) is maintained low enough at all times to ensure sonic flow at the nozzle throat.

The mass $M$ contained at the time $t$ in a reactor cavity of volume $V$ is

$$
V o(t)=v_{\sigma_{0}}-\int_{0}^{t} \frac{d M}{d t} d t,
$$

where the subscript o denotes the initial state following completion of the pellet burn.

Equation (18) implies that

$$
\frac{d o}{d t}=-\frac{1}{V} \frac{d M}{d t}
$$

We also have the relation

$$
\frac{d M}{d t}=A 0^{\star} u^{\star}=A 0^{\star} a^{\star},
$$

where $u$ is the particle velocity, $a$ is the sound speed, $A$ is the throat area, and the asterisk denotes conditions at the throat. For an ideal gas with a constant ratin of specific heats $\gamma$,

$$
o^{*}=o\left(\frac{1+\gamma}{2}\right)^{\frac{1}{1-\gamma}}
$$

and

$$
a^{*}=a \sqrt{\frac{2}{\gamma+T}}=\sqrt{\frac{2 \gamma}{\gamma+T} \frac{p}{0}},
$$

where $p$ is the pressure; in this case, the value of the critical pressure ratio is

$$
\left(\frac{r+1}{2}\right)^{\frac{\gamma}{\gamma-1}}
$$

For an adiabatic process, which we assume,

$$
\frac{\mathrm{p}}{\mathrm{P}_{0}}=\left(\frac{0}{\mathrm{o}_{0}}\right)^{\mathrm{Y}}
$$

Combining Eqs. (19) through (22), we abtain the differential equation

$$
\frac{d o}{d t}=-\frac{A}{v}\left(\frac{2}{T+\gamma}\right)^{\frac{1+\gamma}{2(\gamma-1)}} \sqrt{\frac{\gamma \rho_{0}}{o_{0}^{\gamma}}} o^{\frac{\gamma+1}{2}},
$$

which can be integrated between the limits

$$
0=o_{0} \text { at } t=0
$$

and

$$
0=o_{a} \text { at } t=\tau_{n} \text {, }
$$

where the subscript a denotes the desired ambient value and $\tau_{n}$ is the time required to complete the process.

Using the relation

$$
\frac{D}{p}=\frac{m}{R T} \text {, }
$$

where $m$ is the molecular mass, $R$ is the universal gas constant, and $T$ is the temperature, the result is

$$
\tau_{n}=\frac{V}{A} F(\gamma) \sqrt{\frac{m}{R T_{0}}}\left[\sqrt{\frac{T_{0}}{T_{a}}}-1\right],
$$

with the algebraic function $F(\gamma)$ given by

$$
F(\gamma)=\left(\frac{1}{2}\right)^{\frac{1}{\gamma-1}}(\gamma+1)^{\frac{\gamma+1}{2(\gamma-1)}} \sqrt{\frac{2}{\gamma}} \frac{1}{\gamma-1} .
$$

This function is plotted in Fig. 11; for small values of $r$, the function decreases rapidly with increasing $y$ and approximately levels off near the value 5 for $\gamma>1.50$.

In reactor applications, the ratio of initial postexplosion temperature to ambient temperature, $T_{0} / T_{a}$, probably will be very high (for 


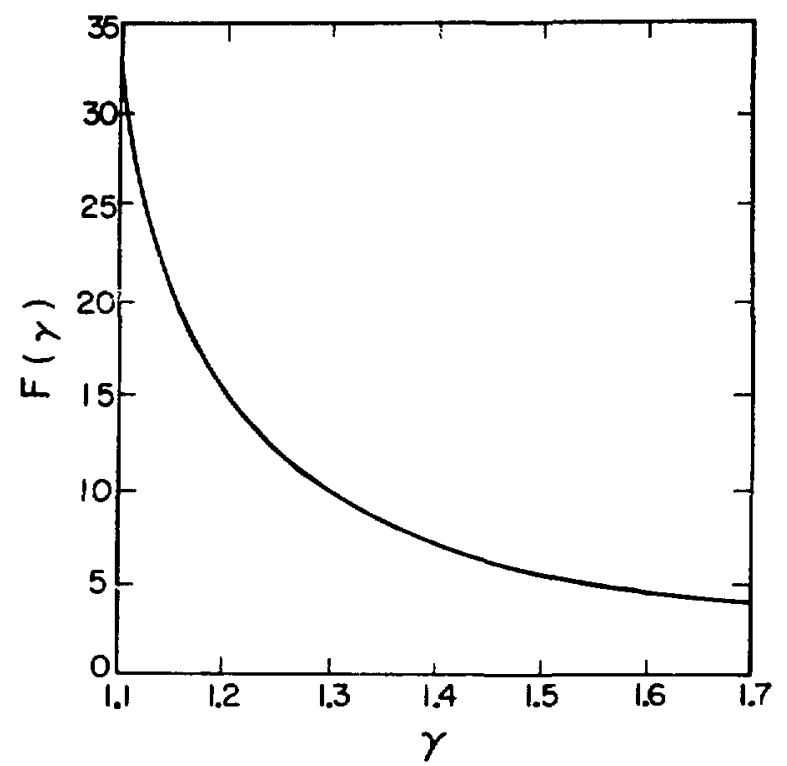

Fig. 11. Effect of the adiabatic exponent $\gamma$ on cavity exhaust time.

example, $\left.T_{0} / T_{a}>100\right)$. In that case, we can neglect 1 in the last term of Eq. (26) and obtain an expression that does not contain the initial conditions attained after completion of the pellet burn but only the ambient temperature that the exhaust process is intended to reach:

$$
\tau_{n}=\frac{v}{A} F(\gamma) \sqrt{\frac{m}{R T_{a}}} .
$$

This result seems to indicate that $\tau_{n}$ does not depend on the initial (postexplosion) conditions, but this is not really so because the original restriction is on $D_{a}$. To determine this quantity from Eq. (28), we must know tice pressure $\mathrm{p}_{\mathrm{a}}$ that is related to the initial pressure $p_{0}$ by the adiabatic expansion process.

Equation (28) shows the expected proportionality of $\tau_{n}$ to the volume of the cavity, $v$, and the inverse proportionality to the nozzle throat area $A$. It also shows that the time raquired to exhaust the cavity increases as the square root of the molecular weight $m$. Therefore, maximizing the frequency of the operation requires the use of a gas with the lowest possible

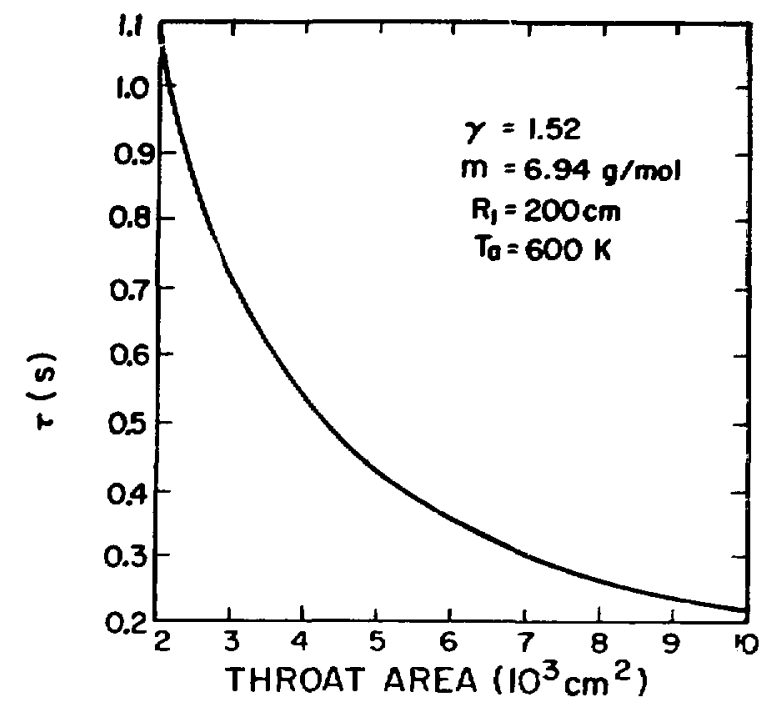

Fig. 12. Dependence of exhaust time on nozzle size, high $\gamma$.

molecular weight, in direct conflict with the requirement to use a high-atomic-number gas as a protective buffer that absorbs the pellet-emitted $x$ rays and slows the debris ions.

To illustrate the time required to exhaust a sphere of $2-m$ radius $\left(V=33.51 \times 10^{6} \mathrm{~cm}^{3}\right)$, Eq. (28) is plotted in Figs. 12 and 13 for high (1.52) and low (1.20) values of $\gamma$ and for $m$ $=6.94 \mathrm{~g} / \mathrm{mole}$ as a function of nozzle throat area

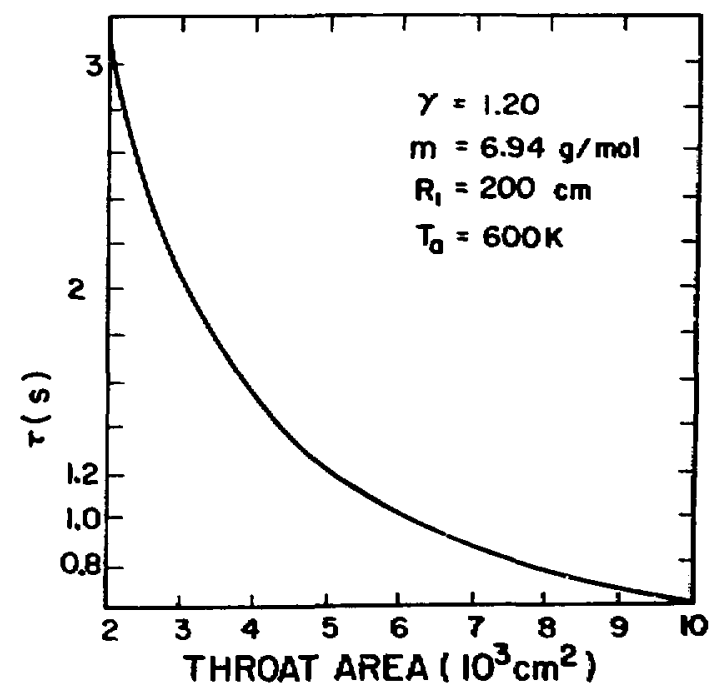

Fig. 13. Dependence of exhaust time on nozzle size, low $\gamma$. 
in the range $2000 \mathrm{~cm}^{2} \leq A \leq 10000 \mathrm{~cm}^{2}$. This range corresponds to nozzle throat radi of 25.23 to $56.42 \mathrm{~cm}$; the corresponding fractions of the surface area occupied by the nozzle throat range from 0.40 to $2.0 \%$. These fractions are small because they are based on the nozzle throat area, but they will increase in proportion to the contraction ratio required for the appropriate nozzle design.

Note that the estimates shown in Figs. 12 and 13 were derived on the basis of choked flow and therefo:e cannot be decreased by the addition of larger capacity pumps. The estimates indicate that the exhaust time will determine the pulse repetition frequency for designs that require a substantial amount of gas in the reacior cavity.

2. Condensation Effects. In reactor cavity designs where liquid lithium serves as first-wall protection (for example, the wetted wall concept), condensation of vapor on the wall is an effective mechanism for mass removal from the cavity. We can easily estimate the rate of mass removal with this mechanism, within the framework of the above formulation, by substituting the condensation rate for the flow through the nozzle. In this case, the analogue of $\mathrm{Fq} .(20)$ is

$$
\frac{d M}{d t}=S\left(\phi_{+}-\phi_{-}\right),
$$

where $S$ is the effective (wetted) wall ared, $\phi_{+}$is the flux of vapor toward the liquid surface, and $\Phi_{-}$is the evaporation rate of the liquid. The sign convention is consistent with that of Eq. (19). An elementary diffusion approximation of the molecular transport process yields the following expressions 20 for the fluxes.

$$
\Phi_{+}=\sigma_{c^{p}} \sqrt{\frac{m}{2 \pi R T}}
$$

and

$$
\phi_{-}=\sigma_{e} p_{v s} \sqrt{\frac{m}{2 \pi R T_{s}}} \text {, }
$$

where $\sigma_{c}$ and $\sigma_{e}$ are condensation and evaporation (accommodation) coefficients, $T_{S}$ is the liquid surface temperature, and $p_{y_{S}}$ is the vapor pressure corresponding to $T_{S}$. The vapor flux $\Phi_{+}$given by Eq. (30) is a conservative estimate because it does not contain the effect of convective velocity toward the wall. In the ICF reactor cavity, such a velocity will be induced by the microexplosion-generated shock wave, and its magnitude will be significant. Because experimental evidence indicates ${ }^{20}$ that ${ }_{0}$ $\tau \mathrm{ce}_{\mathrm{e}} \sim 1$, we omit these coefficients from further analysis.

We now postulate that enough liquid lithium will be maintained and circulated in the reactor cavity to ensure that the inequality

$$
p / \sqrt{T} \gg p_{v s} / \sqrt{T_{s}}
$$

is satisfied at all times; consequently, we may neglect the evaporation rate of the liquid, $\phi$.. We also assume that the vapor remaining inside the cavity behaves as a perfect gas and follows the adiabatic process described by Eq. (22). Then, using Eq. (25), Eq. (29) becomes

$$
\frac{d M}{d t}=S \sqrt{\frac{R_{0}}{2 \pi m_{0}^{\gamma-T}}} 0^{\frac{\gamma+1}{2}} ;
$$

its functional form is identical to that of Eq. (23). Remembering that integration is a linear operation and that $D$ is related to $M$ by Eq. (19), we combine Eq. (23) with Eq. (33) and obtain the following equation to describe the change in density induced by simultaneous condensation and nozzle exhaust.

$\frac{d o}{d t}=-\frac{S}{V} \sqrt{\frac{\mathrm{RT}_{0}}{\mathrm{mo}_{0}^{\gamma-1}}}\left[\frac{1}{\sqrt{2 \pi}}+\frac{A}{S}\left(\frac{2}{\gamma+\gamma}\right)^{\frac{1+\gamma}{2(\gamma-1)}} \sqrt{\gamma}\right] 0^{\frac{\gamma+1}{2}}$.

Equation (34) makes it convenient to compare the rates of mass removal by condensation and by exhaust through a nozzle. The condensation rate 
is represented in the bracket of Eq. (34) by the term $1 / \sqrt{2 \pi}=0.399$ and the exhaust by the area ratio A/S multiplied with a function of $\gamma$ that is plotted in Fig. 14 for all physically relevant values of $r$. The plot shows that it never exceeds the value 0.75 . Therefore, when the assumptions specified in the preceding discussion are satisfied, the mass removal rate through the nozzle is but a fraction of the condensation rate, and the value of the fraction is less than $A / S \sqrt{2 \pi}$. Because for workable reactor vessel designs $A / S$ will not be larger than a few hundreds, it is justifiable to neglect vapor flow through the nozzle in preliminary estimates of the time required for the restoration of ambient cavity conditions. With this approximation, Eq. (34) integrated between the limits given by Eq. (24) yields the following expression for the time ${ }_{C}$ required to exhaust the cavity with the condensation process:

$$
{ }_{c}=\frac{V}{S} \frac{2}{\gamma-T} \sqrt{\frac{2 \pi m}{R T_{0}}}\left[\sqrt{\frac{T_{0}}{T_{a}}}-1\right] .
$$

This result differs from that given by Eq. (26) in the volume-to-area ratios and in the multiplicative functions of $r$ that were shown not to differ grossly. Therefore, as expected,

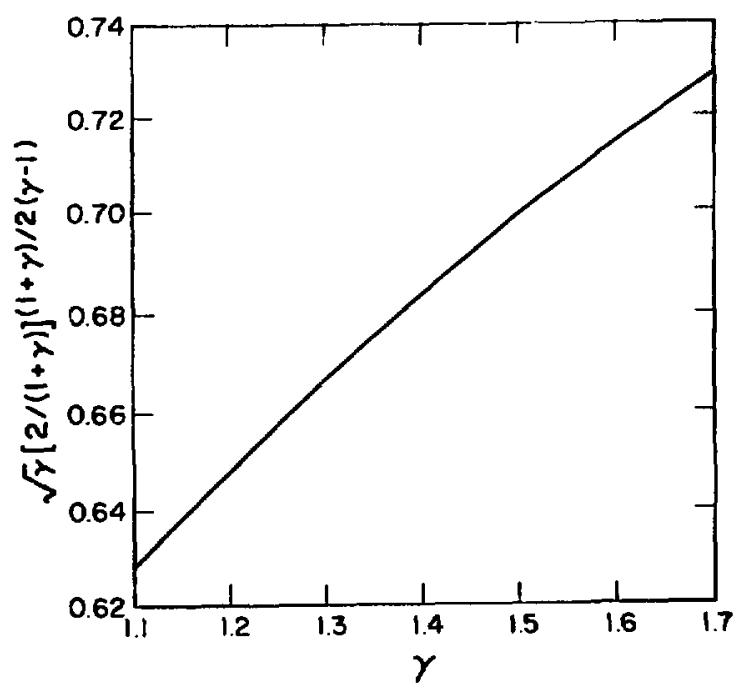

Fig. 14. Effect of adiabatic exponent on exhaust rate. the time required to restore the cavity to ambient conditions by condensation, ${ }^{\top}{ }$, is approximately A/S times shorter than that required for exhausting through a nozzle, $\tau_{n}$; that is, $\tau_{c} / \tau_{n} \approx A / S$. Because the functional forms of Eqs. (26) and (35) are the same, the observations made about $\tau_{n}$ apply also to $\tau_{\text {C. }}$.

\section{FIRST-WALL EFFECTS}

The radiation and debris emitted during fuelpellet burn, modified through interaction with the cavity medium, impinge on the first wall causing temperature increase, material loss, and stress waves. Thermal generation and propagation of stress waves are discussed in Sec. $V$ in connection with load and stress analysis. Temperature increase and material ioss are the topics of the present section.

\section{A. Surface Temperature Increase}

Pulses of energy deposited in ICF reactor walls are characterized by shallow penetration ( $21 \mu \mathrm{m})$ and short duration ( $1 \mathrm{~ns}$ to $1 \mathrm{us}$ ). An analysis of the first-wall heating requires approximations that are valid for shallow depth of energy deposition and for short, intense pulses. 21

1. Surface Flux Model. The most obvious way to approximate wall heating with an energy pulse whose depth of penetration is small relative to the wall thickness is to assume that the depth of penetration is zero and to model the pulse with a specified flux of energy, $F\left(\operatorname{erg} / \mathrm{cm}^{2} \mathrm{~s}\right)$, across the surface. The evolution of temperature distribution inside the wall then is described by the heat conduction equation that, in onedimensional approximation and for constant thermophysical properties, is 22

$$
\frac{\partial T}{\partial t}=\kappa \frac{\partial^{2} T}{\partial x^{2}}
$$

and by the boundary condition 


$$
-K \text { DC } \frac{\partial T}{\partial x}=F \text { at } x=0 \text {, }
$$

where $T$ is temperature, $t$ is time, $x$ is space coordinate perpendicular to the surface, $k$ is thermal diffusivity, $D$ is density, $c$ is heat cepacity, and $F$ is flux of energy per unit surface area per unit time.

A constant temperature distribution usually is specified as the initial condition for the above system, but this is not essential; explicit solutions $c a n$ be obtained for several distributions, 22 and, if resort is made to numerical integration, the initial temperature distribution can be arbitrary.

For a constant initial temperature distribution, which may be assumed to be zero without any loss of generality, the surface temperature at time $T$ (end of the pulse) resulting from the solution of Eqs. (36) and (37) is

$$
T_{A}(0, T)=\frac{2 F}{O C \sqrt{\pi K}} \sqrt{T} \text {. }
$$

This solution frequently has been used 23,24 to calculate first-wall evaporation rates in magnetically confined fusion reactors.

The derivation of Eq. (38) required the postulate that flux $F$ is constant in the interval $0 \leq t \leq \tau$; therefore its value is given by $4 \pi R^{2} F=x Y / \tau$, where $Y$ is the pellet yield and $x$ is the fraction of vield in the energy puise whose effects are being investigated. Substituting this value of $F$ in Eq. (38) results in an expression for the surface temperature increment $T_{A}$ that increases beyond bounus as the pulse duration $T$ tends to zero. Thus, Eq. (38) is not valid for very shori pulses; a quantitative estimate of the range of validity will follow from the volume scurce model and from the analysis presented in the next section.

2. Volume Source Model. To improve the above modei, we replace the surface flux $F$ with a volumetric energy deposition $s$ extending to a depth $\delta$. In that case, the temperature evolu$t$ ion is governed by the equation

$$
\frac{\partial T}{\partial t}=\kappa \frac{\partial^{2} T}{\partial x^{2}}+\frac{s}{\partial c}
$$

and by the boundary condition

$$
\frac{\partial T}{\partial x}=0 \text { at } x=0,
$$

where $s$ is the rate of energy deposition per unit volume per unit time.

For the volume source rpecified by

$s=\left\{\begin{array}{l}s_{0}, 0<t \leq \tau, 0 \leq x \leq \delta \\ 0, \text { otherwise }\end{array}\right.$,

the surface temperature increase (for an initially constant temperature) obtained from the solution of Eqs. (39) and (40) is 25

$T_{B}(0, \tau)=\frac{S_{0} \tau}{D C}\left[\operatorname{erf} u-2 u^{2} \operatorname{erfc} u+\frac{2}{\sqrt{\pi}} u e^{-u^{2}}\right]$,

where

$$
\begin{aligned}
& s_{0}=\text { constant, } \\
& \delta=\text { depth of energy deposition, and } \\
& u=\delta / 2 \sqrt{k T} .
\end{aligned}
$$

Equation (41) can be simplified significantly when the nondimensional variable charactoristic of heat transfer $u$ is either smali or large. For smail $u$, that is, $u \leq 0.75$, linear terms in power series exparsions approximate exponential and error functions to within a few per cent. In this case.

$$
T_{B}(0, \tau)=\frac{4 S_{0} T U}{0 . \sqrt{\pi}}
$$

Because for the pulse energy $x y$ the constant source strength is $4 \pi R^{2} s_{0}=x y / \delta \tau$, Ea. (42) is identical with Eq. (38); that is, predictions based on these two models agree. Clearly, the surface temperature increase is dominated by the neat conductivity of the wall material.

For large $u$, that is, $u \geq 1.75$, erf(u) $\approx 1, \quad \operatorname{erfc}(u) \approx 0, \quad$ and $\quad \exp \left(-u^{2}\right) \approx 0 ; \quad$ therefore,

$$
T_{B}(0, T)=\frac{S_{O} T}{O C}
$$

The surface temperature increase is dominated by heat capacity, and the resuit expressed in 
Eq. (43) could be derived naively by equating the heat stored in a spherical shell of thickness $\delta$ (cm) to the pulse energy $x y$. It is reassuring to know that this naive result is also the limiting value of a rigorous general solution of the heat transfer equation. 25

Because Eq. (43) is indeperident of the pulse duration $T$, the surface temperature $T_{B}(0, \tau)$ does not increase indefinitely as the pulse duration tends to zero; however, it does so when the depth of energy depositon $\delta$ tends to zero. Thus we have exchanged the singular behavior with respect to pulse length for singular behavior with respect to the depth of energy deposition.

For $u$ in the intermediate range, that is, $0.75<u<1.75$, surface temperature increase should be determined by Eq. (41). Comparisons of temperature increases predicted for stainless steel by Eqs. (38) and (41) are shown in Fig. 15 for $F=\tau^{-1}\left(\mathrm{~J} / \mathrm{cm}^{2} \mathrm{~s}\right)$ and $\delta=5 \mathrm{\mu m}$. The plots illustrate the different behaviar of the two solutions for short pulses. The surface flux model predicts that the surface temperature will increase as $\tau^{-1 / 2}$, and the volume source mode? predicts it will approach the value fi/oco. which is constant for fluxes inversely proportional to the pulse duration.

The comparison between the surface flux and the volume source models can be generalized by calculating the normalized difference $\left(T_{A}\right.$ - $\left.\mathrm{T}_{B}\right) / \mathrm{T}_{\mathrm{B}}$ for increasing values of $u$. Because the power series expansion of Eq. (41) yields $\left(T_{A}-T_{B}\right) / T_{A}=u+0\left(u^{2}\right)$, the discrepancy between the two modeis is equal to the value of the characteristic variable u for smail $u$. The exact relationship is plotted in Fig. 16 .

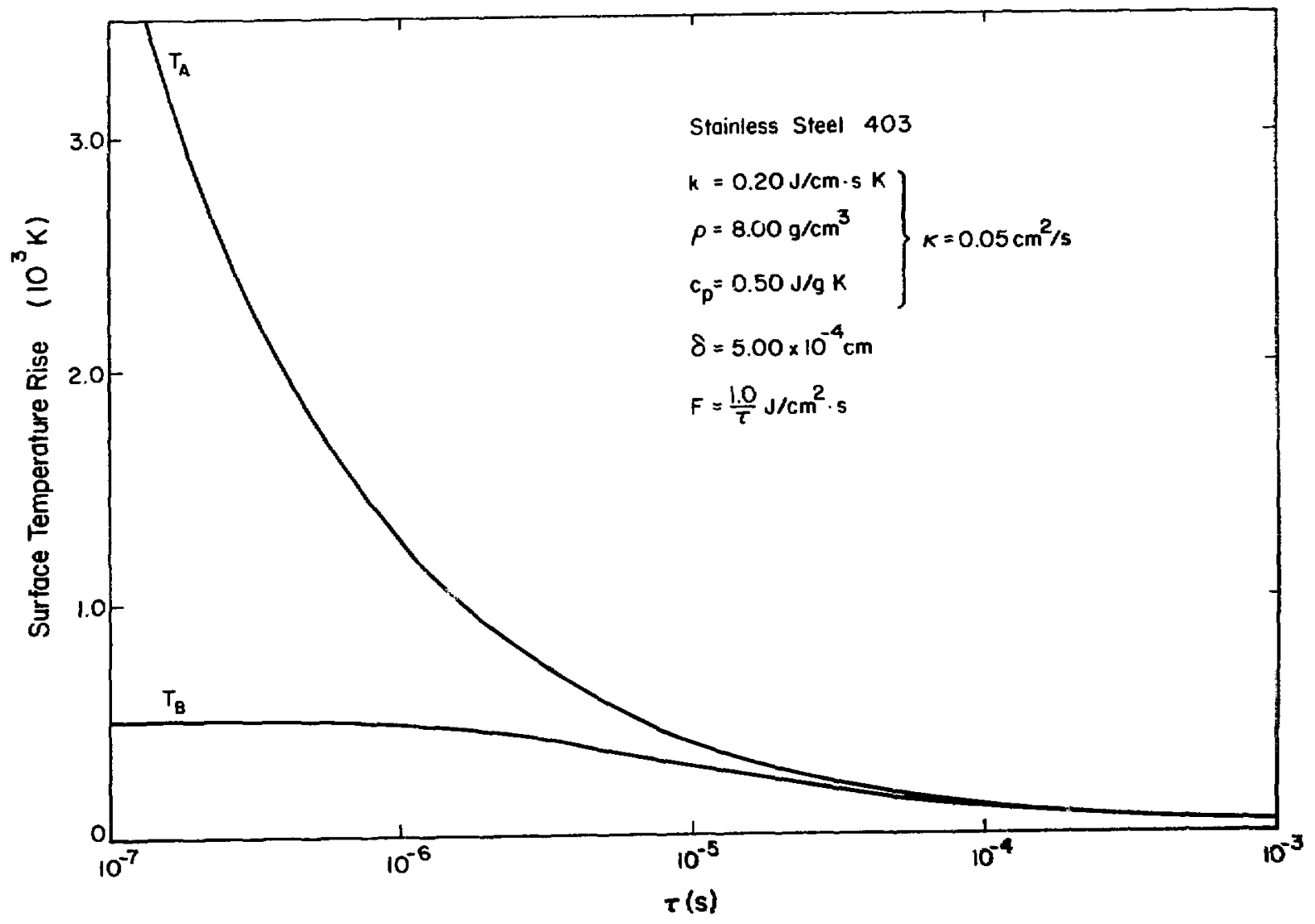

Fig. 15. Surface temperature rise for stainless steel calculated with surface fiux (TA) and volume source $\left(T_{B}\right)$ models. 


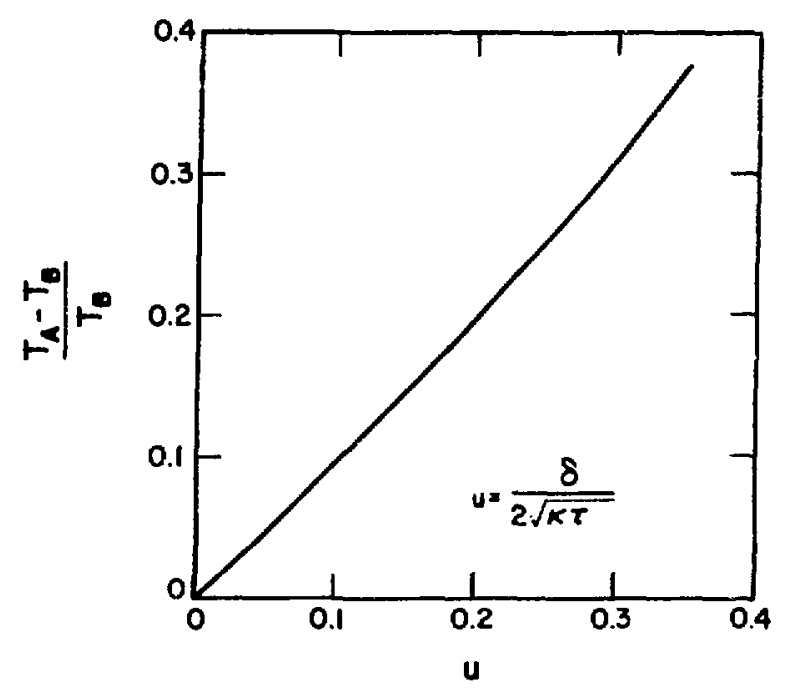

Fig. 16. Normalized difference between surface temperature rises obtained from surface flux and volume source niodels.

Present estimates of energy deposition in ICF reactor walls by $x$ ray's and charged debris particles indicate thermal loadings consisting of several pulses, some of which may overlap in time and be short enough to fall into the regime where surface flux models are not sufficiently accurate. The wall response to such thermal loading can be analyzed by using the superposition principle to generalize the volume source model, Eq. (39), to a source composed of many putses; that is, by setting

$$
s=\sum_{i=1}^{N} s_{i}
$$

with

$$
s_{i}=s_{0 i} \text { for } 0 \leq x \leq \delta_{i}, \quad t_{i 1} \leq t \leq t_{i 2}
$$

and

$$
s_{i}=0 \text { otherwise. }
$$

Figure 17 shows a source composed of two pulses. The first pulse is $0.1 \mu \mathrm{s}$ long, penetrates to a depth of $5.8 \mu \mathrm{m}$, and has a source strength of $6 \times 10^{9} \mathrm{~J} / \mathrm{cm}^{3} \mathrm{~s}$. The second pulse begins $0.5 \mu \mathrm{s}$ after termination of the first pulse, lasts $0.6 \mu \mathrm{s}$, penetrates to a depth of

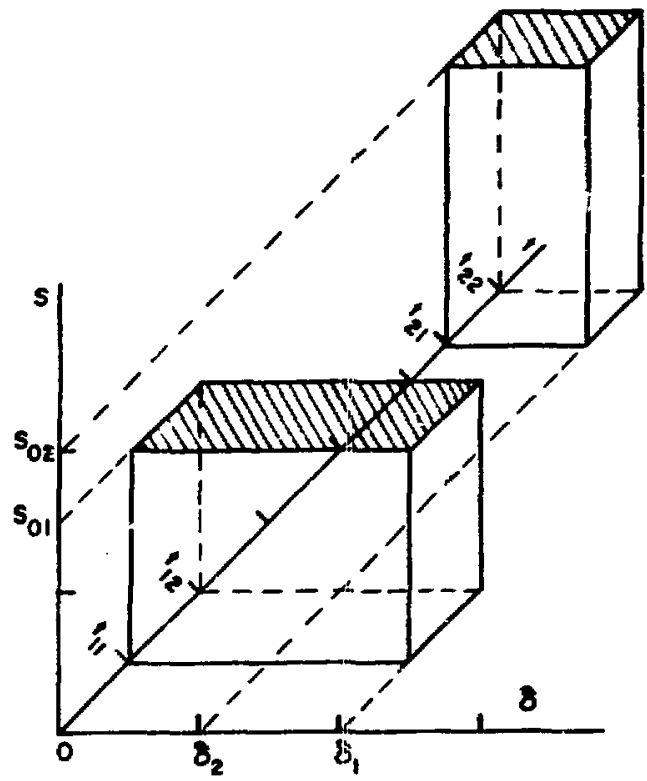

Fig. 17. Volume source composed of two pillses.

$0.677 \mu \mathrm{m}$, and has a scurce strength of 1.71 $\times 10^{70} \mathrm{~J} / \mathrm{cm}^{3} \mathrm{~s}$.

The surface temperature history of a moiybdenum wall subjected to these two pulses is shown in Fig. 18. It demonstrates the cooling between the pulses and the rapid temperature decrease at the end of the second pulse that is due to the good thermal conductivity of molybdenum. The temperatures predicted by the surface flux model at the end of each pulse also are indicated. As expected, the two predictions for the first pulse, for which $u=1.41$, are very far apart. For the second pulse, $u=0.07$, and the fractionai surface temperature difference is about $9 \%$, consistent with the result presented in Fig. 16. Show in Fig. 19 are the temperature distributions inside the wall that correspond to the surface temperature history in Fig. 18.

The solution presented above has been obtained with the Laplace transform technique. A general solution of the same problem also has been obtained by conn and coworkers ${ }^{4}$ using orthogonal function expansions. Both analytic approaches become very cumbersome when the number of pulses in the source tem exceeds three or more.

We emphasize that the solutions and their behavior are valid only as long as the thermophysical properties (for example, heat conductivity and heat capacityl of the will aterial 


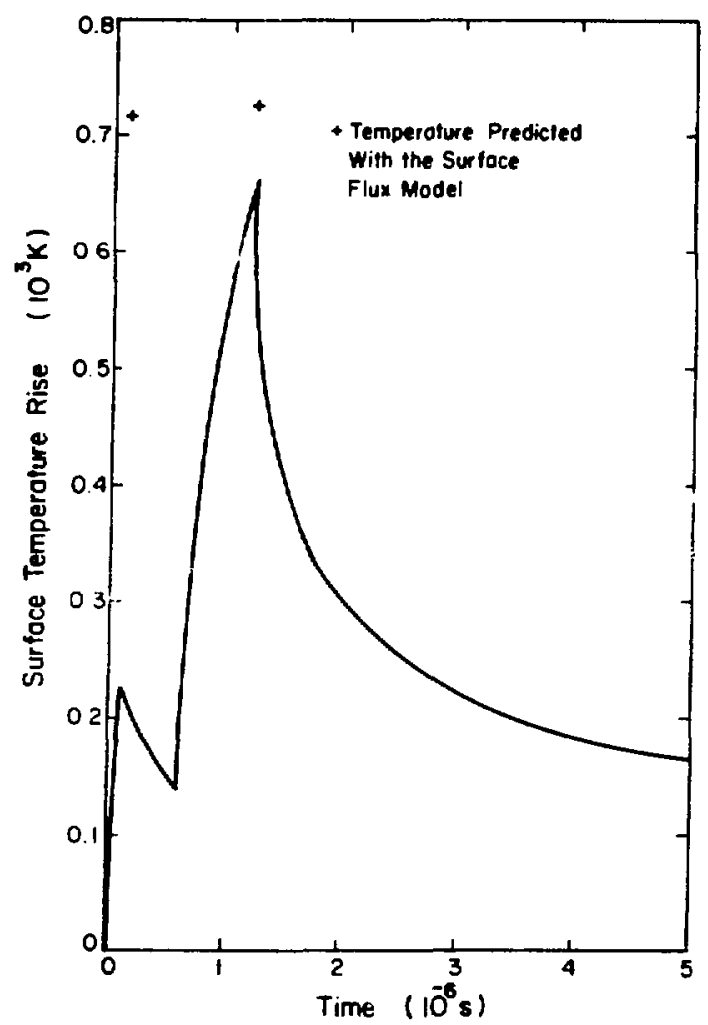

Fig. 1 . Surface temperature rise in response to two pulses.

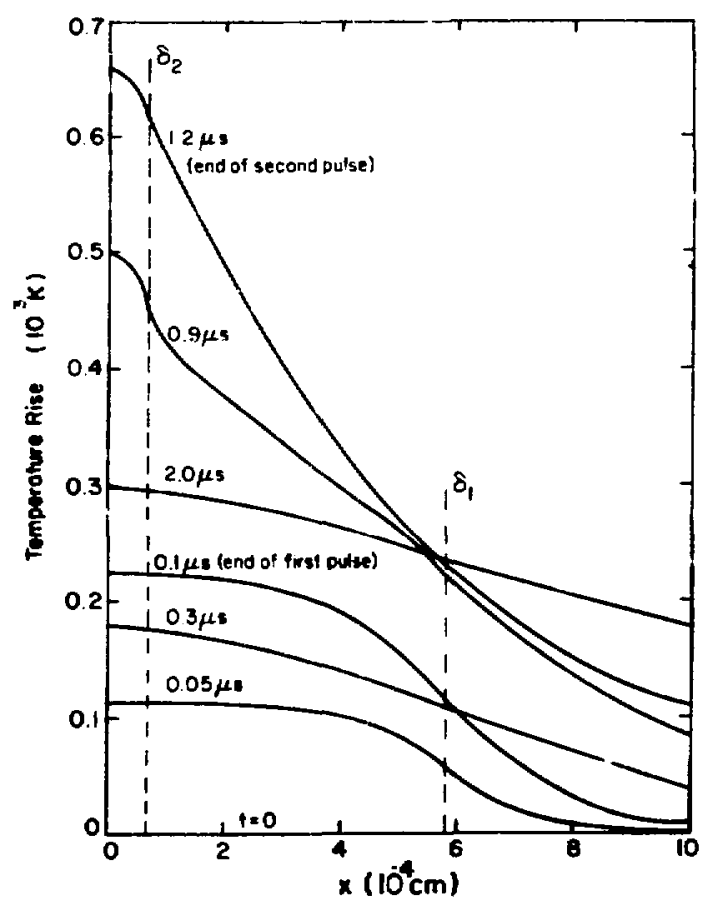

Fig. 19. Temperature distributions inside cavity wall inducer by thermal loading of two pulses. remain constant. If this is not a satisfactory approximation, explicit soiutions cannot be found, and the governing equations must be integrated numerically.

B. Material Loss

Loss of material from the ICF reactor cavity wall is caused by two physical mechanisms: (1) evaporaticn or sublimation (therme1) and (2) erosion (mechanical).

1. Evaporation. To encure economically acceptable lifetimes, ICF reactor vessel walls should last a substantial length of time. This requirement constrains thermal loadings to values that do not induce melting of the material. Even if melting occurs locally, the pulsed loads are too short for developing flow on a macroscopic scale. Under such i:onditions, the evaporation and sublimation proces'ses are physically indistinguishable and can lie described with one analytic expression.

A convenient. and sufficiently accurate expression is obtuined from the Langmuir quasiequilibrium evaporation theory. In this theory, the diffusive transport of the vapor awy from the surface is approximated with the equilibrium value determined for the vapor pressure at surface temperature, and the transport toward the surface (condensation) is neglected. The vapor pressure is specified with the Clausius-Clapeyron expression valid for low values of that quantity.

With these approximations, the rate of evaporation of surface material per unit area, $E_{v r}$, is given by

$$
E_{v r}=\frac{\sigma \sqrt{m}}{\sqrt{2 \pi R T}} B e^{-H / R T},
$$

where $\sigma$ is an experimentally determined surface accominodation coefficient, $m$ is the molecular weight, $R$ is the universal gas constant, $H$ is the heat of vaporization, and 8 is a constant determined from empirical vapor pressure data. The total emount of material evaporated during time $T$ lusualiy $T$ is the pulse or that fart of the cycle during wich the wall is at elevated zemperaturel is given by 


$$
E_{v}=\int_{0}^{T} E_{v r}(t) d t
$$

Before discussing a procedure for evaluating Eqs. (45) and (46), $i$ is useful to examine the dependence of the evaporation rate on surface temperature; an estimete of that dependence is necessary to motivate the procedure.

The expression for the evaporation rate, Eq. (45), contains as a multiplier the exponential function with; a negative and numerically very 1. - exponent that changes in inverse proportion to .ite temperature. Consequently, it is very sensitive to the surface temprature. For example, the ratio of evaporation rates for carbon at surface temperatures of 2000 and $3000 \mathrm{~K}$ is $e^{-14.16}$ or $0.71 \times 10^{-6}$. Because of this sensitivity, the evaluation of the evaporation rate with Eq. (45) and of total evaporation with Eq. (46) requires a very accurate $d^{+}$ermination of surface temperature history. Because the evaporation rate usual" is significant at eievated temperatures at which the therraphys.cal properties of the material are affected, the use of constant values for these quantities during the evolution of the temperature distribution is unacceptable.

Therefore, an accurate determination of evaporative material loss reauires that the temperature be calculated from the generalization of Ea. (39) to variable coefficients: that is, from

$$
o c(T) \frac{\partial T}{\partial t}=\frac{\partial}{\partial x}\left[k(T) \frac{\partial T}{\partial x}\right]+s(x, t)
$$

(k is the heat conductivity), supplemented with appropriate boundary and initial conditions. The source function $s(x, t)$ is determined from numerical calculations of energy deposition profiles for each component of the energy pulse.

The presence of variable coefficients and of a variable forcing function in Eq. (47), in general, precludes an analytic soiution and requires numerical integration. In this integration it is convenient to translate the space grid continuously in such a way as to maintain a constant amount of mass between the source and each grid point: that is, use the Lagrangian mesh $o \Delta x$. Because of this procedure, the material evaporated during the early part of the pulse remains in the computational mesh and shields the solid surface during the later part of the pulse: therefore. it is not necessary to exolicitly consider the changes in density ithe density dependence is not indicated in Eq. (47)1. This approximation is valid as long as the effect of expansion velocity is negligible compared to the thermal effects. The drnamic effects are discussed in Sec. $v$ in connection with the analysis of stress waves.

The necessity for numerical integrations in calculating evaporative mass losses and the extreme sensitivity of the results to initial conditions and material properties imply that analyses of this ohenomenun must be specific with respect to both fuel-dellet and reactor-cavity designs.

$\frac{\text { 2. Sputter }}{\frac{\text { ment Fu Erosion in Inertial Confine- }}{\text { Introduction. Explosive or near- }}}$ exDlosive combustion of D-T fuel pellets in ICF reactors produces high-energy neutrons and plasma dobris. The debris is composed of fast-traveling light and heavy ions; the heavy jons are obtained fron the disintegration of structural shelis that enclose fuel pellets. These energetic particles strike reactor cavity walls and transfer their momentum to the surface or subsurface atoms. When the amount of momentum transferred exceecs surface and bulk bonas, the atoms of the solid wall are eroded or sputtered away.

in this section we discuss the phenonenology of sputtering in general terms and show how the theoretically and experimentally acquired knowledge of this damage mechanism can be applied in enoineering desion calculations. We develop analvtical estimates that show explicitly the depencence of erosion on fuel-dellet mass and yield and on the materials of the pellet shell and reactor wall. A computational orocedure for detailed exact calculation of sputtering erosion a) so is included.

b. General Description. When an energetic ion strikes the surface of a solid, it gives up its momentum to the surface and subsurface atoms surrounding the Doint of impact. An atom of the solid near the surface may acquire sufficient momentum in the perpendicular direction to break the surface bond and escape. This phenomenon is known as sputtering: it is mechanical as opposed to thermal evaporation or sublimation. 
Sputtering is encountered in many technologies. For example, aerospace vehicles may be damaged by sputtering caused by impinging interDlanetary dust particies, or cathodes may be eroded by ions of the electric arc. In controlled themonuclear fusion, sputtering causes concern for two reasons. In magnetic confinement reactors, sputtering may introduce sufficient amounts of impurities into the plasma to inhibit or even prevent efficient burn. In inertial conffnement reactors. pellet microexplosions generate energetic diasma debris that may cause considerable surface erosion.

The denetration depth of the impinging ions is small comoared to the structural dimensions of reactor walls: therefore, the surface atoms in most cases escade in the direction from which the projectile ions arrive. This nechanism sometimes is called backward sputtering. Some impinging particles, however, may have sufficient energy to penetrate the solid and to escape on the opposite side. taking along the surrounding atoms. This phenomenon is termed forward or transmission sputtering. In ICF applications, only neutrons induce forward sputtering. Dur analysis is limited to backward sputtering because damage from forward neutron souttering is negligible compared to other neutron damage mechanisms.

jouttring is describen by the sputtering coefficient $S$, defined as the number of atoms knocked out of the solid surface by one projectile ion. This definition is used in theoretical calculations of $S$ that take into account collision eneray and cross section. crystal lattice st-ucture, and surface and bulk energy bonds. 26-28

However, it is not possible to perform an experiment corresponding to the above definition. Therefore, sputtering coefficients are determined by bombarding a surface with an ion beam of specified intensity $n$ (ions/s) and measuring the amount cf sputtered material after a given length of time $t$. In this process, effects are present that are not accounted for in the theoretical definition of S: for example, modification of the surface properties by the implanted ions or redeposition of sputtered ions. Because the actual environment more closely resembles experimental conditions than the theoretical definition of $S$, theoretical determinations of sputtering coefficients are of limited value in practical calculatioris. They should be used only when experimental data are lacking or when they are needed to supplement inadequate experimental data. Later indicate how the theoretical results are used in our work.

c. Dependence of Sputtering and Its Apgroximation. The amount of material sputteried from a surface depends on the mass, charge, efiergy, and angle of incidence of the striking ions, as well as on target properties, such is surface temperature and finish. The dependence on each of these parameters usually is determined by varying them in a series of experiments in wich the remaining factors are kept constant.

Effects of surface temperature and roughness have nnt been investigated systematicaliy. Data on the iffect of carget temperature on sputtering appear inconclusive and mostly 1 imited to temperature recordings during investigations of other effects. 29-31 Also, there is some evidence that the observed temperature-induced change in sputtering yield may be due to thermal evaporation 32 or chemical removal. 33

The effect of surface deviations from a plane on sputtering yield has been reported in only one reference. 34 In view of this lack of information, we postulate that the sputtering cuefficient $S$ is independent of both target surface temperature and finish. The discussion of our approach will show that these effects can be incorporated easily into the analysis when adeçuate and reliable data become available.

Results of sputtering experiments customarily are reported as graphs or tables showing the dependence of sputtering yield on either ion energy or angle of incidence for a given pair of ion and target materials. It is natural, therefore, to postulate that the sputtering coefficient $S$ is in the form of a product of two factors: one describing its dependence on inn energy, and the other on ion angle of incidence. Accordingly, for a given ion-target combination, we set

$$
S(E, \theta)=S_{1}(E) S_{2}(\theta)
$$

where $E$ denotes the energy and $\theta$ is the angle of ion incidence measured from the normal to the surface. 
The dependence of sputtering on the energy of the striking ions is the most extensively studied aspect of the phenomenon. The literature is too vast to discuss here; the data obtained have been summarized and reviewed recently by Carter and Colligan $^{29}$ and by Behrisch. ${ }^{24}$ The following dependence emerged from the numerous investigations. 29 There is a threshold energy $E_{0}$ below which no sputtering occurs. Above $E_{0}, S_{1}$ rises gradually, becomes nearly linear with $E$ in a certain range, reaches a maximum, and decreases asymptotically to zero as $\ell n E / E$. This behavior is illustrated schematically in Fig. 20. It is physically plausible because $E_{0}$ is related to the energy required to break both the bulk and the surface bonds and because the decrease beyond maximum is due to the increasing depth of penetration of energetic ions, which makes it difficult for the effects to propagate back to the surface before dissipating.

The behavior of $S_{1}$ depicted in Fig. 20 can be described by

$$
s_{1}=0, \quad \varepsilon<1
$$

and

$$
S_{1}=S_{0} \frac{(\ln \varepsilon)^{1+c / \varepsilon}}{\varepsilon^{1+b / \varepsilon}}, \quad \varepsilon \geq 1,
$$

where $E$ is $E / E_{0}$ and $S_{0}, b$, and $c$ are positive constants to be determined from empirical data. When sufficient data points are available (more than 5 to 10 ), the constants $S_{0}, b$, and $c$ can be calculated to approximate most of the experimentally determined dependencies to within a few per cent. Agreement with particular data is

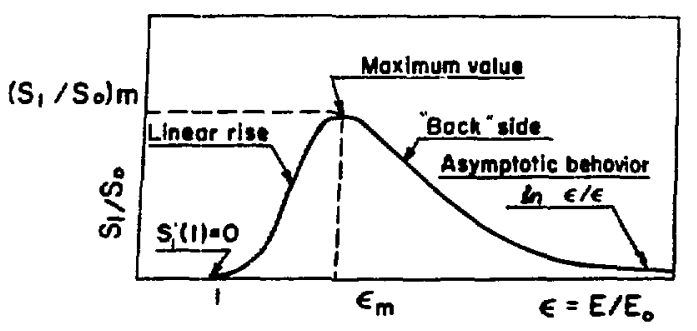

demonstrated in the next section. However, when only a few experimental points are available, the determination of coefficients is not reliable and should be supplemented with physico-theoretical considerations.

In addition to approximating the experimental data, Eq. (49) correctly describes the asymptotic behavior of the sputtering coefficient at large energies. This behavior has been determined theoretically 26-29 to be $l n \varepsilon / \epsilon$, and it is the limiting form of Eq. (49) as $E \rightarrow \infty$. A1so, $S_{1}$ (E) given by Eq. (49) (with $b>0, c>0$ ) has a vanishing slope at the threshold energy $\varepsilon=1$ and for $\varepsilon \rightarrow \infty$, as the experimental evidence seems to suggest.

The effect of the angle of incidence $\theta$ on the sputtering coefficient $S_{2}$ has been studied by many investigators. 24,29,35-37 They found that the reciprocal of $\cos \theta$ generally reflects the behavior of $S_{2}(\theta)$, but in some cases a negative exponent different from unity better approximates the data. Cleariy, such an increasing behavior can persist only to a maximm value of $S_{2}$, which occurs for $\theta$ in the neighborhood of 60 to $80^{\circ}$; after the maximum is attained, $S_{2}(\theta)$ must vanish at $\theta=90^{\circ}$ because sputtering cannot be induced by ions traveling parallel to the surface. Thus $S_{2}(\theta)$ must start from unity at $\theta=0$ (by definition) with initially horizontal slope, increase to a maximum before $\theta=90^{\circ}$, and vanish precipitously at $\theta=90^{\circ}$. A function with these properties is given by

$$
S_{2}=\left[\exp \left(g x^{2}\right)\left(1-x^{2}\right)\right]^{h}
$$

where $x=2 \theta / \pi$ and the parameters $g$ and $h$ specify the location $x_{m}$ and magnitude $S_{2 n}$ of the maximum value of $S_{2}(\theta)$. In terms of these guantities, $g$ and $h$ are given by

$$
g=\frac{1}{1-x_{m}^{2}}
$$

and

$$
h=\frac{\ln S_{2 m}}{9 x_{m}^{2}+\ln \left(1-x_{m}^{2}\right)} .
$$


For specific computations, $x_{m}$ and $S_{2 m}$ are chosen from experimental data. The angular dependence $S_{2}(\theta)$ given by Eq. (50) is shown in Fig. 21 for $S_{2 m}=2.5$ at $\theta_{m}=75^{\circ}$ with the reciprocal cosine relation and experimental data from Ref. 37.

d. Determination of Constants. In our studies, the dependence of the sputtering coefficient on ion energy $S_{1}(E)$ given by Eq. (49) is determined in ejther of two ways. When sufficient empirica? data are available, the constants $S_{0}$, $b$, and $c$ are calculated to obtain the least squares approximation to given experimental points. When the data are insufficient, we begin with a set of values for $S_{0}, b$, and $c$ obtained for some similar ion-target pair and vary them until the curve approximates the available data and its maximum occurs sufficiently near the theoretically predicted location of the maximum. (Locations of theoretically and experimentally determined sputtering maxima agree much better than the amplitudes of the maxima.) The results of Goldman and Simon, 26 Sigmund, 27 or Kanaya et a1. 28 are used in this process. The ability to change the approximation and determination of its acceptability is left to our judgment. The above procedure also is used when Eq. (48) with regressively determined coefficients has multiple

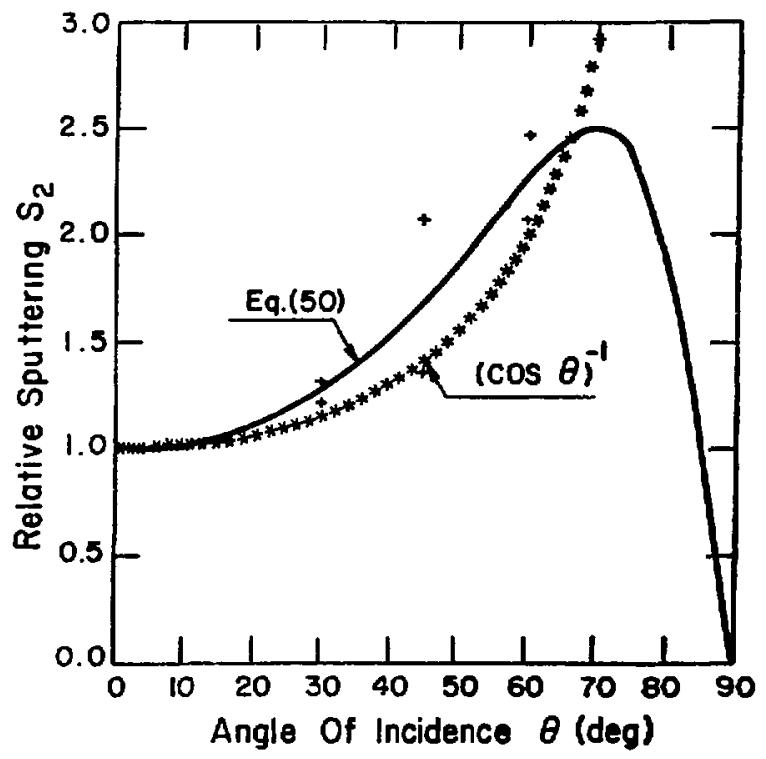

Fig. 21. Dependence of sputtering yield on angle of incidence. maxima; this occurs rarely, but we were unable to exclude analytically ranges of coefficients where it occurs.

The dependence of the sputtering coefficient on the ion angle of incidence $s_{2}(\theta)$ given by Eq. (50) can be determined also in two ways. Either the constants $g$ and $h$ are calculated from Eq. (51) and from direct specification of the parameters $x_{m}$ and $S_{2 m}$, or $g$ and $h$ are determined from a least squares approximation to available data.

e. Validity of the Approximation. Equations (49) and (50), which constitute our sputtering model, describe the correct qualitative variation of sputtering yield with ion energy and angle of incidence. Now we demonstrate the quantitative agreement of these expressions with theoretical and experimental results.

Figure 22 shows $S_{1}(E)$ for the bombardment of niobium with alpha particles [given by Eq. (49)] in which the constants $S_{0}, b$, and $c$ have been determined with a least squares regression to experimental data (indicated by crosses) reported by Carter and Colligan, 29 Yonts, 31 Rosenberg and Wehner, 34 Sommers et al., 37 and Kaminsky. 38 Agreement appears to be within experimental error. The dashed curve in Fig. 22 is the theoretical dependence derived by Goldman

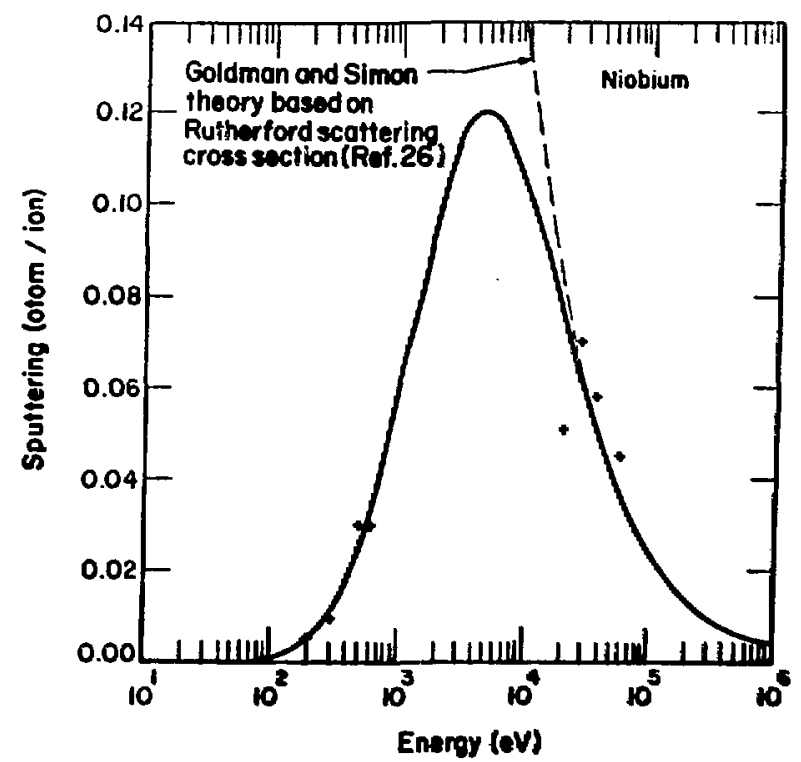

Fig. 22. Sputtering yield of niobium bombarded with $\alpha$ particles. 
and Simon ${ }^{26}$ based on the assumption of Rutherford scattering cross sections, which is a good approximation at high energies. The two curves are identical in the range in which the GoldmanSimon theory is valid; this agreement is real because the Goldman-Simon expression for sputtering has no adjustable parameters.

The maximum sputtering yield indicated by Eq. (49) is 0.12 atom/ion at $4750 \mathrm{eV}$. Theoretical modeling of the sputtering phenomenon 27,28 indicates a maximum of 0.237 atom/ion at $3864 \mathrm{ev}$. Such correspondence is reasonable in view of the discrepancy between theoretical definition and experimental determinations of sputtering mentioned earlier.

Figures 23 and 24 show sputtering yield for iron and carbon bombarded with alpha particles predicted by Eq. (49), with coefficients determined from experimental results (indicated by crosses) reported by Carter and colligan, ${ }^{29}$ .osenberg and Wehner, ${ }^{34}$ and Kulcinski et a1. ${ }^{39}$ Again, agreement is excellent. However, the agreement with asymptotic behavior predicted by the analysis of Goldman and $\operatorname{Simon}^{26}$ is not as good as in the case of niobium, probably because for iron and carbon we had no data points for the high-energy "back" of the sputtering curve.

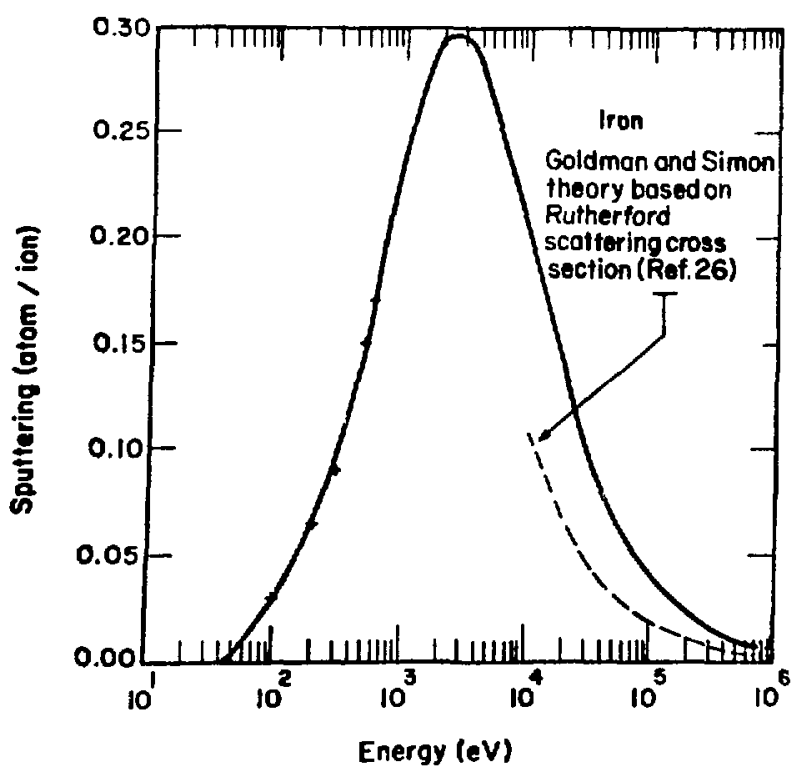

Fig. 23. Sputtering yield of iron bombarded with a particles.

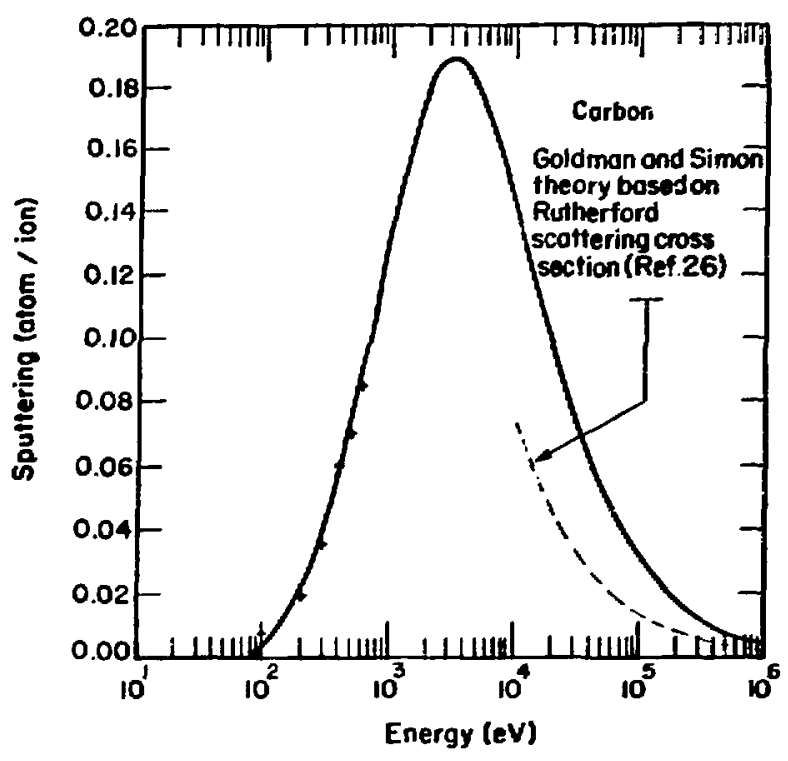

Fig. 24. Sputtering yield of carbon bombarded with a particles.

Equation (49) predicts a maximum sputtering of almost 0.30 atom/ion at $3000 \mathrm{el}$ for iron and of 0.19 atom/ion at $3000 \mathrm{eV}$ for carbon. Theoretical analyses of sigmund 27 and Kanaya et a). ${ }^{28}$ predict a maximum of 0.482 atom/ion at $2207 \mathrm{eV}$ for iron and of 0.129 atom/ion at $514 \mathrm{eV}$ for carbon. In addition to the difference between theoretical and experimental sputtering, there are uncertainties about the type of carbon used in different experiments and its lattice orientation (if lattices existed) during ion bombardment; therefore, comparing theory and experiment for this material may not be meaningful.

These three calculations illustrate how well Eq. (49) approximates experimental data over the entire range of energy values.

The approximation by Eq. (50) of the angular dependence of sputtering is compared in Fig. 21 with experimental data and with the usually postulated reciprocal cosine relation; agreement is satisfactory. Because of insufficient empirical and theoretical results, this topic is not discussed further.

f. Erosion Rate and Erosion. For feasibility studies, preliminary design analyses, and other engineering applications, it is more convenient to know surface erosion than the sputtering coefficient itself. Surface erosion is determined 
by integrating the product of the sputtering coefficient and the ion flux over all energies and angles of incidence to obtain the erosion rate and then integrating the rate with respect to time. Thus, with $S(E, \theta)$ given by EqS. (48), (49), and (50), the erosion rate $E_{r r}$ is given by

$$
E_{r r}=\frac{a}{A} \bar{D} \iint S(E, \theta) n(E, \theta, t) d E d \theta \text {, }
$$

where $a$ is the atomic weight, $A$ is Avogadro's number, and $\mathcal{O}$ is density of the target material.

The factor a/Ao converts the units of erosion rate $E_{r r}$ from atoms/s to $\mathrm{cm}^{3} / \mathrm{s}$, which are more appropriate for engineering analyses. In some parametric studies, it is convenient to interpret the quantity $n(E, \theta, t)$ as ion flux per unit area (ions/s $\mathrm{cm}^{2}$ ), so that the units of the erosion rate are $\mathrm{cm} / \mathrm{s}$, and $E_{r r}$ is a direct indication of the rate at which the surface recedes because of sputtering. For other applications, it may be convenient to aiso convert $n$ into mass flux with the factor a/A appropriate for ions and thus to combine the effects of pellet mass and pulse repetition rate into one parameter.

Total erosion after any given length of time $\tau$ is given by

$$
E_{r}=\int_{0}^{T} E_{r r}(t) d t .
$$

g. Dependence of Sputtering Erosion on FuelPellet Characteristics, A simple Version of $S_{f}(E)$. For parametric investigations of sputtering erosion, we use an approximate version of $S_{1}(E)$ that is sufficiently simple to allow analytic representation of the sputtering yield $E_{r}$. To derive this representation, we assume no angle of incidence dependence $\left[S_{2}(\theta) \rightrightarrows 1\right.$, to be justified in the next section] and postulate that the sputtering coefficient depends on the energy of the impinging ion in the following way.

- $S(E)$ increases i inearly from zero at $E=0$ to $S_{m}$ at $E_{m}$, where $S_{m}$ is the maximum value of $S$ and $E_{m}$ is the ion energy at which sputtering reaches its maximum.

- $S(E)$ remains constant in the region $E_{m}$ $\leq E \leq r E_{m}$, where $r \geq e=2.718$ is an arbitrary constant (the restriction on $r$ is not essential and is explained later).
- $S(E)$ decreases as ene/E for $E$ $>r E_{m}$, where now $\varepsilon=E / E_{m}$.

This behavior is represented schematically in Fig. 25. It is a reasonable approximation of the theoretically and experimentally determined functional dependence of $S(E) .29$ We assumed that the threshold energy for sputtering is zero. This approximation is satisfactory for the present analysis because the threshold energy is usually $10 \mathrm{eV}$ and therefore negligible in comparison to $E_{m}$, which generally equals tens or hundreds of kev.

The physical quantities $E_{m}$ and $S_{m}$ are characteristic of the ion material and deperid on its atomic number $z$. He use sigmund's theor $y^{27}$ to calculate that dependence. The results for molybdenum targets (cavity walls) are snown in Figs. 26 and 27; they indicate that $E_{m}(Z)$ is nearly quadratic and $S_{m}(Z)$ neariy linear in $Z$. Sigmund's results calculated for other target materials have similar behavior as shown by the plots of the same quantities for a carbon wall in Figs. 28 and 29. Accordingly, we set

$$
E_{m}=k z^{2}
$$

and

$$
S_{m}=C_{1}+C_{2} Z \text {, }
$$

where the constants $K, C_{1}$, and $C_{2}$ are obtained by matching Eqs. (54) and (55) with iheoretically or experimentally obtained dependencies $E_{m}(Z)$ and $S_{m}(Z)$. For example, in the case of a molybdenum target (cavity wall), Figs. 26 and 27 indicate $k \approx 9000, C_{1} \approx 2.0$, and $C_{2} \approx 0.2$.

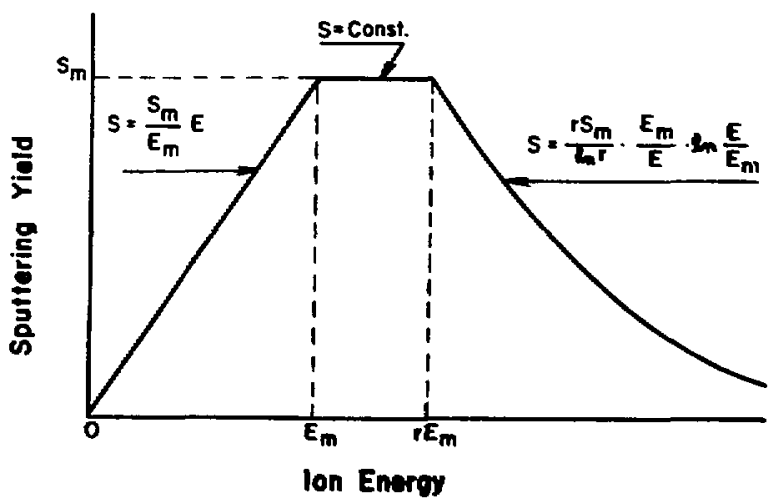

Fig. 25. Approximate energy dependence of the sputtering coefficient $S(E)$. 

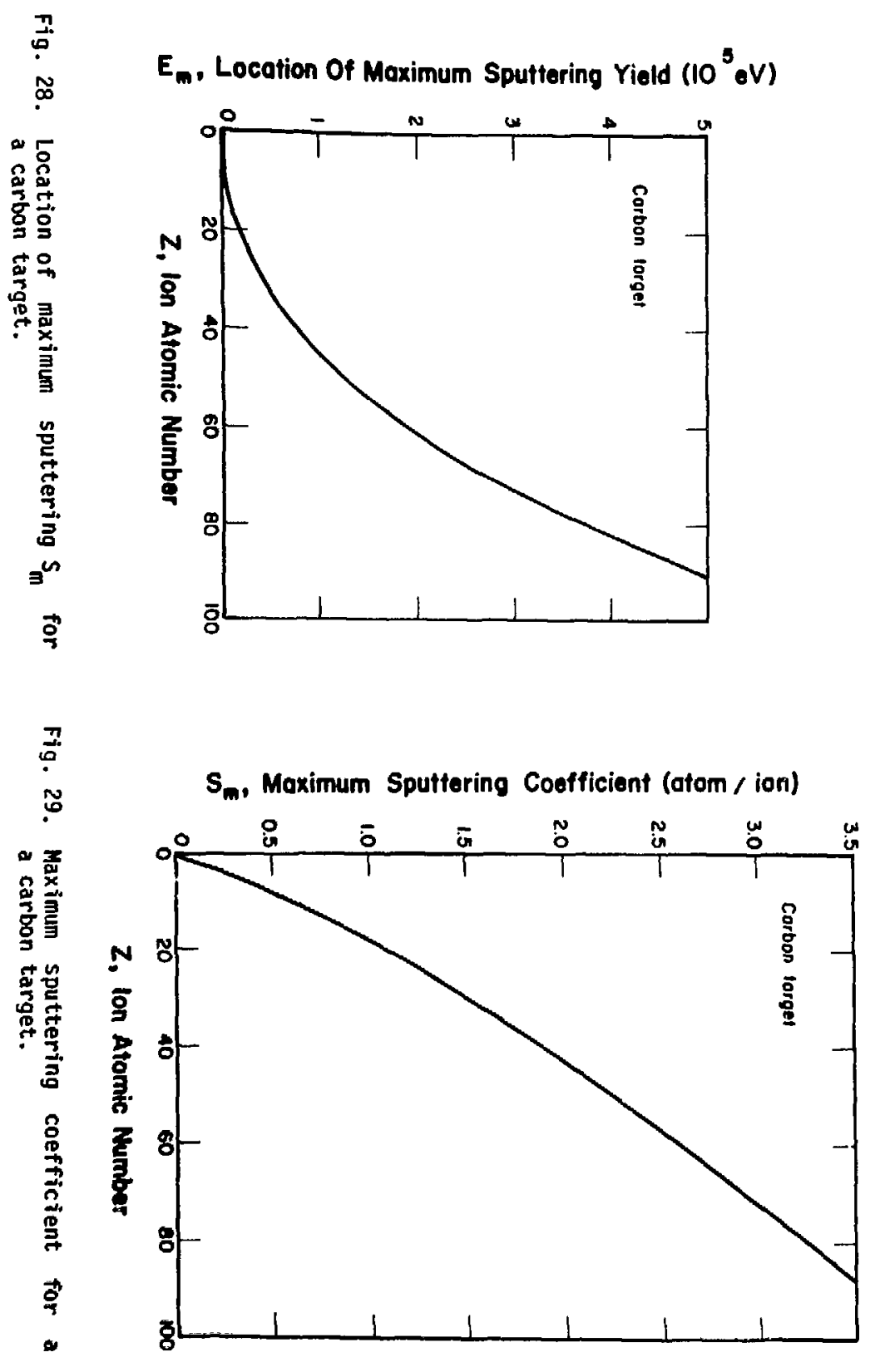

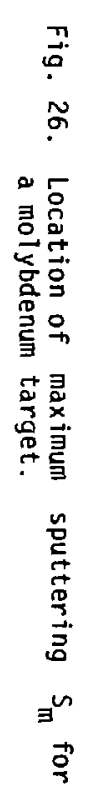

$E_{m}$, Location Of Maximum Sputtering Yield $\left(10^{5} \mathrm{eV}\right)$
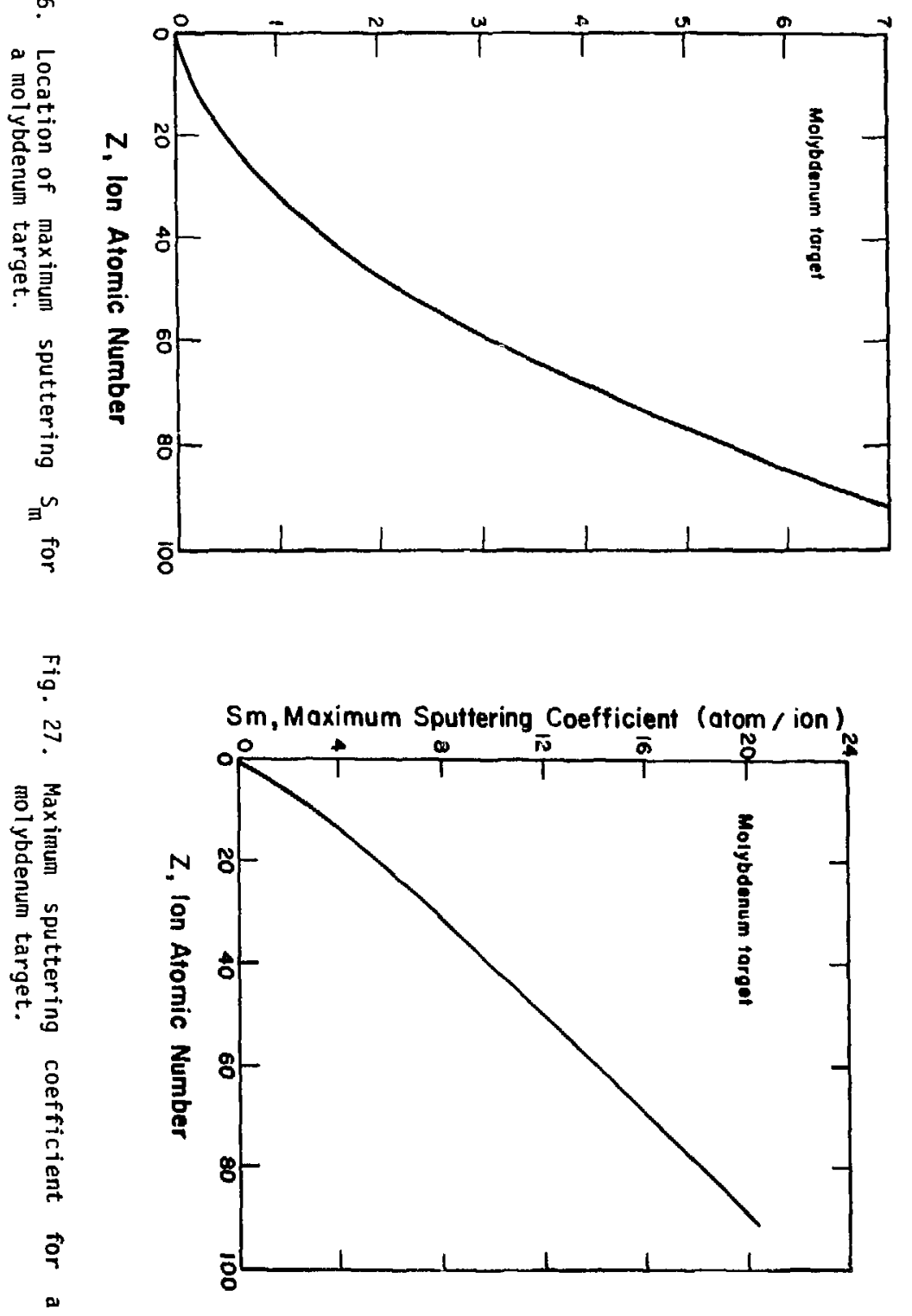
Consistent with the approximations inherent in Eqs. (54) and (55), we also approximate the atomic weight a by

$$
a=2 z+\delta z^{2},
$$

where o' is a small number.

Equations (55) and (56) indicate that, as $Z$ increases, the atomic weight increases slightly faster than the maximum of the sputtering coefficient $S_{m}$. Therefore, sputtering erosion per gram (instead of per ion) of the pellet material should decrease as the atomic number of the dominant pollet material increases. That this is indeed so is shown in Fig. 30, where total erosion of the wall per gram of pellet material is plotted as a function of ion energy for light $(\alpha)$ and heavy (U) ions and two wall materials, iron (steel) and carbon. Sigmund's theoretical model of sputtering yield ${ }^{27}$ was used to calculate these results.

h. Erosion Estimates. At present, the composition and design of ICF fuel pellets have not been determined. Therefore, for reactor feasibility and systems studies, it is convenient to have expressions that estimate the amount of erosion per pellet microexplosion in terms of pellet material, mass $M$, and energy yield.

To derive such expressions, we assume that pellet composition can be characterized with adequate accuracy by an average atomic weight. Then

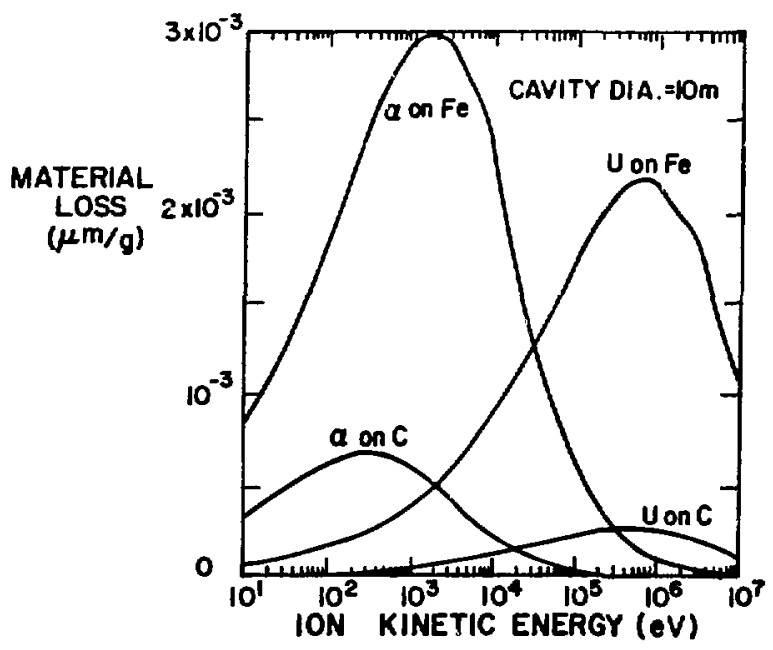

Fig. 30. Sputtering erosion per unit mass of pellet material. the number of plasma ions generated by its microexplosion is

$$
n_{0}=\frac{M A}{a} \text {. }
$$

If we assume that the fusion energy is distributed uniformly over all ions, the ayerage energy per ion $E$ is

$$
E=\frac{f Y a}{\text { MA }} \text {, }
$$

where $Y$ is the total microexplosion energy re lease in electron volts $(\mathrm{eV})$ and $f$ is the fraction of yield deposited in plasma debris.

In this simp?e case, Eq. (53) for the total erosion reduces to

$$
E_{r}=S n_{0}
$$

To evaluate Eq. (59), we use the simple version of $S(E)$ introduced previously and shown in Fig. 25 and consider separately the three ion energy regimes: low, intormediate, and high (where the sputtering coefficient increases, remains constant, and decreases, respectively).

i. Low-Energy Regime, $E<E_{m}$. Plasma ions will be in the low-energy regime when the inequality (fYa/MA) $\leq E_{m}$ is satisfied; in general, this inequality is satisfied when the pellet yield-to-mass ratio is low.

In this regime, the sputtering coefficient increases linearly with ion energy and is given by $S=\left(S_{m} / E_{m}\right) E$. Using this expression together with Eqs. (57) and (58) in Eq. (59), we obtain

$$
E_{r}=\frac{S_{m}}{E_{m}} f Y,
$$

which becomes, upon substitution for $E_{m}$ and $S_{\pi}$, expressions given by Eqs. (54) and (55),

$$
E_{r}=\frac{c_{1}+c_{2} z}{k z^{2}} f y
$$

This result shows that in the low-eneryy regime (where the sputtering coefficient increases with ion energy), the total anount of material eroded is independent of pellet mass, directly proportional to the total ion energy, and for a constant 
pellet yield decreases as the atomic number $z$ of the pellet material increases.

j. Intermediate-Energy Regime, $E_{m}<E$ $\leq r E_{m}$. In this regime, the ion energy satis-
fies $\leq r E_{m}$, and the sputtering coefficient is constant with ion energy. The amount of material eroded is given by

$$
E_{r}=S_{m} \frac{M A}{a} \text {. }
$$

Using Eqs. (55) and (56) to express $E_{r}$ in terms of atomic number, we obtain

$$
E_{r}=\frac{M A}{Z} \frac{C_{1}+C_{2} Z}{2+\delta Z} \text {. }
$$

This result shows that in the intermediate-energy regime (where the sputtering coefficient is independent of ion energy), the amount of eroded material does not depend directly on fuel-pellet yield, is proportional to pellet mass $M$, and for constant $M$ decreases with increasing $Z$. The decrease, however, may not be uniform for some combinations of values of $c_{1}, c_{2}$, and $\delta$.

$$
\text { k. High-Energy Regime, } E>r E_{m} \text {. Plasma }
$$
ions are in the high-energy regime when the inequality (fYa/MA) >rE $m$ is satisfied; in general, this inequality is satisfied when the pellet yield-to-mass ratio is high.

Theoretical and experimental investigations indicate 27,29 that in the high-energy regime, the sputtering coefficient decreases with increasing energy as $\varepsilon^{-1}$ ln $\varepsilon$ (this function decreases monotonically for $\varepsilon \geq e$, hence the prevfous restriction on $r$ ); consequently,

$$
S=\frac{r S_{m}}{\ln r} \frac{\ln \varepsilon}{\varepsilon},
$$

where the multiplicative constant was determined from the continuity of $S$ at $E=r E_{m}$.

Substituting Eq. (64) for S, together with Egs. (57) and (58), into Eq. (59), we obtain

$$
E_{r}=\frac{r(M A)^{2}}{f Y l n r} \frac{E_{m} S}{a^{2}} \ln \frac{f Y a}{W A E_{m}} .
$$

In Eq. (65), the product $E_{m} S_{m}$ increases as $z^{3}$ [Eqs. (54) and (55)], and $a^{2}$ increases as $z^{2}$ [Eq. (56)]; however, no convenient estimate exists to reflect the variation of the logarithmic term with $Z$. The following considerations indicate the behavior of $E_{r}$. For low values of $Z, E_{m}$ is relatively small, making the argument of the logarithm large and the logarithm itself slowly varying with $Z$. Thus, the behavior of $E_{r}$ is determined predominantly by the factor $E_{m} S_{m} / a^{2}$, which increases with $z$. For high values of $Z$, the argument of the logarithm decreases as $(z)^{-1}$, and the logarithm diminishes more rapidly than the increasing factor $E_{m} S_{m} / a^{2}$. Thus, eventually $E_{r}$ decreases with increasing $Z$.

To verify the above deduced behavior and to obtain the value of $Z$ at which $E_{r}$ changes from an increasing to a decreasing function of $Z$, we evaluated Eq. (65) numerically using for $E_{m}(Z)$ and $S_{f}(z)$ exact expressions from Sigmund's theory ${ }^{27}$ and for $a(Z)$ exact values from the periodic table of elements. The calculation was carried out for $r=2.80, f=0.20, Y=100 \mathrm{MJ}$, and $M=0.07 \mathrm{~g}$, chosen to put ions in the highenergy regime for $Z \leq 48$.

The result is plotted in Fig. 31. It shows that $E_{r}$ behaves as predicted: initially it

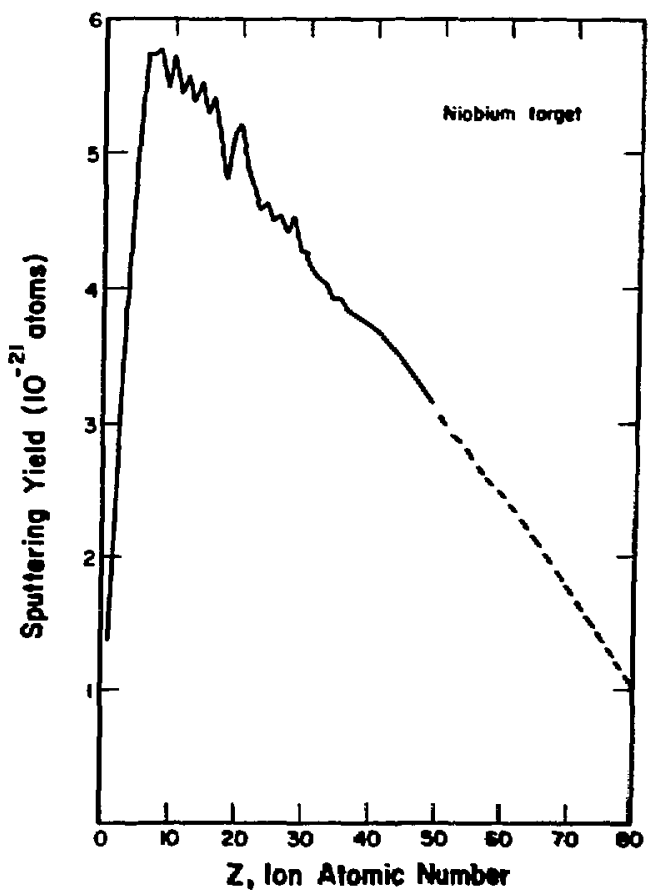

Fig. 31. Variation of total sputtering erosion in the high-energy regime. 
increases with $Z$, reaches a maximum (at $Z=8$ for the particular set of parameter values used in this computation), and then decreases as $Z$ increases. The curve is extended with dashes beyond $Z=49$ to illustrate the treno. The behavior is not uniformly smooth, however, but has small undulations superimposed on the general trend near the maximum.

The complenentary problem of determining the amounts of different wall materials eroded by a given ion flux has been investigated extensively by Wehner and his collaborators, ${ }^{40-42}$ and their findings are summarized in Sec. 7.4.2 of Ref. 29. Briefly, with minor exceptions the sputtering $y$ ield follows closely the state of electron concentrations in the " $d$ " shells of target material atoms and also crudely resembles the reciprocals of the heats of sublimation. Thus, the yield variec almost periodically with target atomic number. The results presented in convenient-to-use graphs can be found in Ref. 29 (PF. 315-316) and are not reproduced here.

1. Dependence of Erosion Rate on Ion-Zeam Characteristics. We conclude this discussion of sputtering with a brief study of the dependence of wall erosion rate on ion-beam characteristics and with a demonstration that the effect of angular dependence of the sputtering coefficient $c$ an be neglected in preliminary studies.

We use Eqs. (49) and (52) to calculate the dependence of erosion rate $E_{r r}$ on ion flux characteristics, and we specify a steady-state flux intensity in the form $n_{1}(E) n_{2}(\theta)$.

To isolate and investigate the effects of ion energy, we set $n_{2}(\theta) \equiv 1$ and specify $n_{1}(E)$ to be a Maxwellian distribution given by

$$
n_{1}(E)=2 n_{0}\left(2 E_{m}\right)^{-3 / 2}\left[E / \pi \exp \left(-E / 2 E_{m}\right)\right]^{1 / 2}
$$

Equation (66) is used only in the interval between the threshold energy $E_{0}$ and some high cutoff value (typically, $10 \mathrm{MeV}$ ), which must ise finite for numerical evaluation of the integral in Eq. (52).

Calculations were performed for an ion beam of peak intensity $8 \times 10^{13}$ alpha particles per $\mathrm{cm}^{2} / \mathrm{s}$. Figure 32 shows the erosion rate as a function of peak entrgy for an ion beam with Maxwellian energy distribution incidint normally on

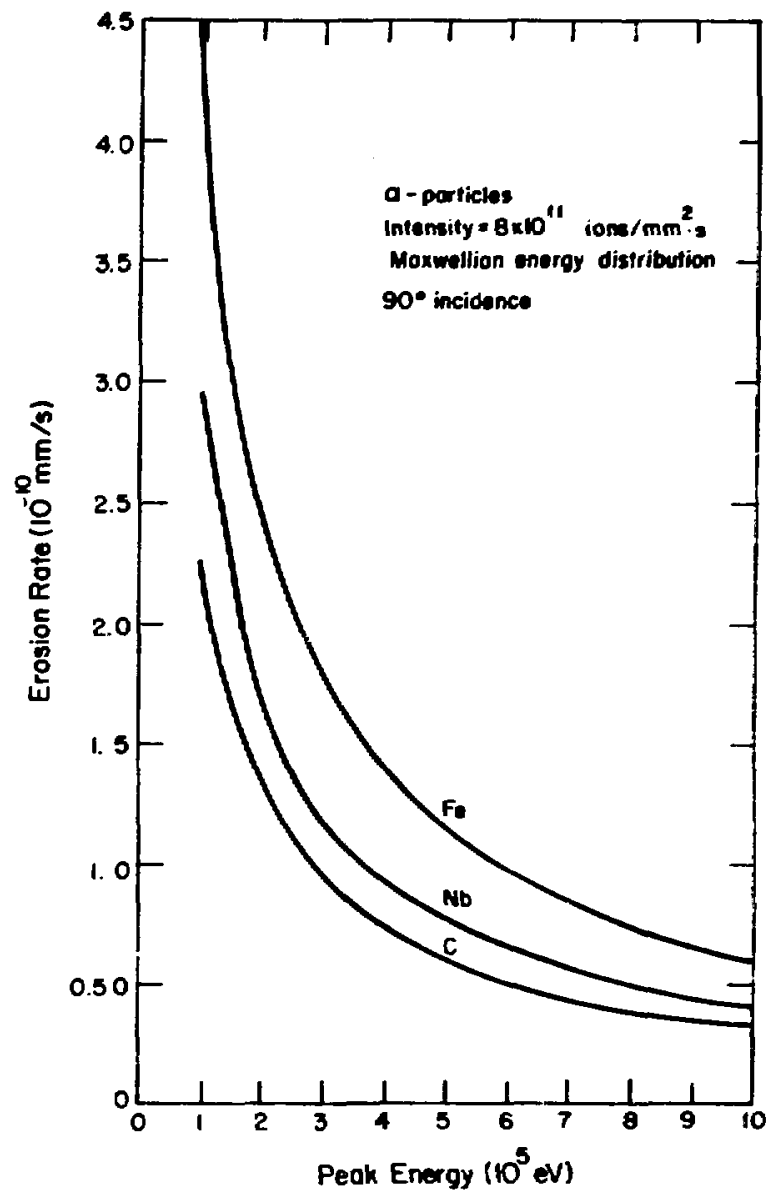

Fig. 32. Energy dependence of erosion rate.

iron, niobium, and carbon targets. The erosion rate decreases rapidly with increasing energy because the energy range included in this graph is on the back side of the sputtering curve (see Fig. 20).

To isolate and investigate the effects of the ion angle of incidence, we set $n_{1}(E)=n_{0}$ (constant) and postulate that the beam intensity in different directions varies as $\cos \theta$ in the interval $0 \leq \theta \leq \pi ; 2$; that is,

$$
n_{2}(\theta)=\frac{\cos \left(\theta-\theta_{p}\right)}{\cos \theta_{p}+\sin \theta_{p}},
$$

where $\theta_{p}$ is the location of the maximum intensity (also in the interval $0 \leq \theta_{p} \leq \pi / 2$ ), and the denominator is the normalizing factor that makes $n_{2}(\theta)$ a probability distribution. 


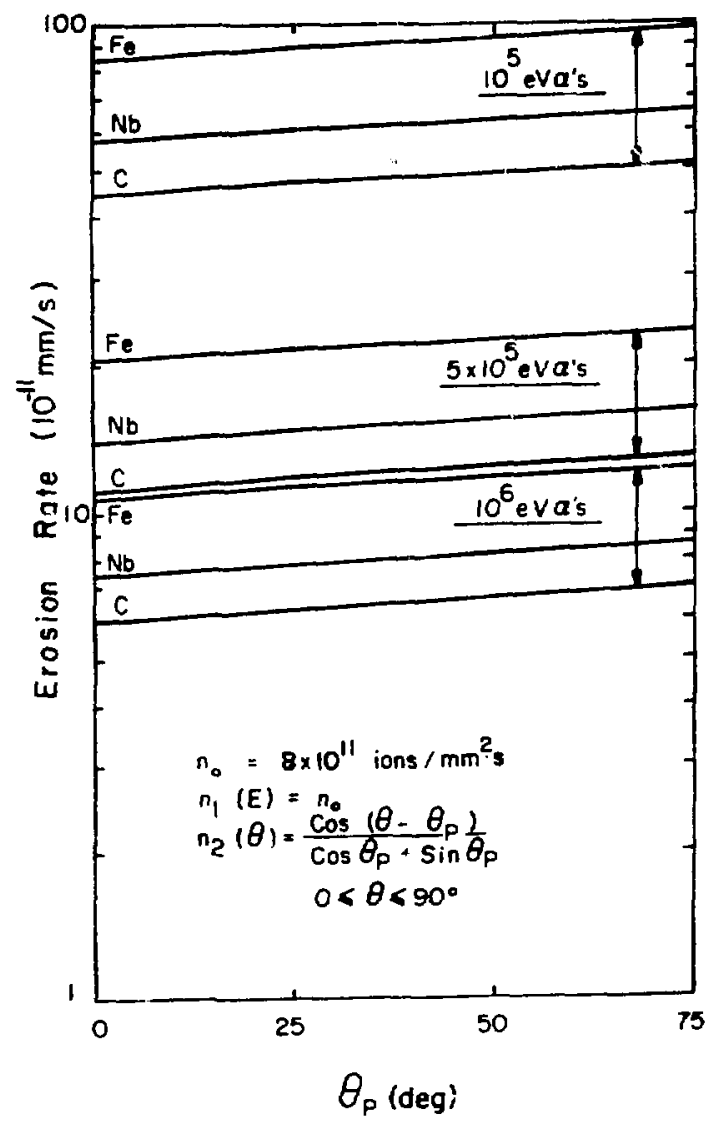

Fig. 33. Angle-of-incidence dependence of erssion rate.

Results of calculations for different materials bombarded with beams of different energy are shown in $\mathrm{Fig} .33$. In the range of angles from 0 to $75^{\circ}$, the variation is small, not exceeding $25 \%$; in these calculations, the sputtering coefficient $S_{2}$ has a maximum of 2.5 located at $75^{\circ}$. Thus, it appears that in preliminary analyses of reactor cavity configurations, the effects of the angle of incidence need not be considered.

\section{ICF REACTOR NEUTRONICS}

\section{A. Introduction}

The energy released from the fuel $(D-T)$ in the pellet during its explosive burn appears as the kinetic energy ( $14 \mathrm{MeV}$ ) of neutrons $(a p-$ proximately 65 to $80 \%$ ), ionized pellet debris (approximately 20 to $30 \%$ ), and $x$ rays. Because most of the energy is in neutron kinetic energy, and it is not known at present how to utilize it directly, every functional ICF reactor must contain a component that

- converts neutron kinetic energy intoconveniently usable form and

- produces ("breeds") tritium in a quantity that equals or exceeds the amount consumid; because tritiun does not occur in nature. breeding is necessary to ensure seifsufficiency of the fusion power generation industry.

Both needs are satisfied by surrounding the source of neutrons with a "blanket" of lithium (liquid or solid compounds). The light element lithium converts neutron kinetic energy into thermal energy by scattering interactions, and tritium is produced by transmutation in a mijor fraction of reactions between neutrons and iltnium atoms. Some of these reactions are exoergic and produce additional thermal energy. It is essential that as much of the neutron energy as possible be converted into highi-grade thermal energy for ultinate conversion into electicicity and that at 'east as much tritium be generated as is consumed and list.

The energy of neitrons enitted from the fuel burning at the core of the pellet is modified as the neutrons pass through the unburned compresseo fuel and the expanding pellet structure. Consequently, the spectrum of the neutron energy that arrives at the containment wall and passes into the blanket may be controlled to some degree ty the pellet design; in a detailed determination of first-wall effects and blanket phenomena, the fuel pellet and the containment vessel shourd be viewed as a system. Such details, however, are feyond the scope of the present discussion.

In th's section, we discuss neutron transport and its calculation, neutron energy conversion and the liquid blanket response, tritium breeding, malerials effects, and an approach to parametric studies.

\section{B. Neutron Transport}

The range of neutron penetration is iarge; therefore, neutrons produce noticeable effects throughowt the blanket and the unsthielded reactor structure. These effects are of two $k$ inds, 
- energy deposition through elastic and inelastic collisions (neutron thermalization) and

- neutron reactions; reactions involving neutrons are important because of ancillary effects such as energy releases and element transmutations that are both beneficial (tritiuin production) and detrimental (for example, helium bubble formation in structurai materials).

Determination of neutronic effects requires integration of the neutron transport equation with coefficients depending on the neutron energy and on the material through which the neutrons pass. Therefore, in addition to time, the equation involves three independent variables: neutron energy, direction of transport, and position. Depending on the treatment of these variables, a variety of approximate methods has been developed for integrating the transport equation. Division of the energy variable into discrete intervals or groups is called the multigroup approximation, whereas that of direction is called the discrete ordinate $\left(S_{n}\right)$ approximation. It is customary and therefore convenient to approximate the variation with the scattering angle by an expansion in Legendre polynomials $P_{n}$ with $n$ denoting the degree of the expansion. These approximations of individual variables may be used in combinations most suitable for the specific problems. However, with the adveni of superfast computers, the expansions into series of orthogonal polynomials are being neglected in favor of totally discrete calculations. For detailed discussions, the reader is referred to textbooks and many excellent expositions of this subject. 43,44

For initial scoping, a one-dimensional, timeindependent $S_{n}$ calculation usually suffices and saves considerable time and computational labor. Typically, the calculation is carried out to order $S_{4}$ and $P_{3} \quad\left(P_{0}\right.$ represents isotropic scattering); for greater accuracy the $S_{8} P_{3}$ approximation may be used.

often a two-dimensional calculation is required (for example, to represent cylindrical geometry). For this purpose a two-dimensional $S_{n}$ code may be used, although the computational time will increase significantly. If, in addition, a time-dependent anaiysis is desired, Monte Carlo is the preferred approach.

$$
\text { The Monte Carlo computationa] }
$$

approach $43,45,46$ follows the paths of individual neutrons by using experimentally or theoretically determined probabilities to determine their fate after each encounter. The number of neutron paths followed must be sufficiently large to ensure that the statistically determined results approximate the exact solution with satisfactory accuracy. This method is advantageous for the determination of neutron transport througn complex and intricate regions.

This discussion is a brief indication of available approaches; the retails of each method may be found in many standard treatises and texts on this subject. Every practitioner of the trade has a favored computational method and a computer code to implement it.

\section{Energy Deposition and Blanket Response}

The $k$ inetic energy of neutrons is transferred to blanket and structural materials primarily through elastic and inelastic scattering encounters and secondarily through exoergic neutron reartions. Because of the second contribution, the heat content of the blariket may exceed the available neutron energy. The ratio of these two quantities is sonetimes called the blanket energy multiplication factor or blanket gain.

Because characterizations of neutron encounters resulting in energy transfer to blanket and structural materials are contained implicitly in the neutron transport equation, its solutions provide energy deposition distributions (or profiles if one-dimensional approximation is used). Neutron energy deposition in liquid lithium regions results in increased temperature and pressure. Because of energy deposition gradients in the lithium, pressure waves result that travel between structural components. An accurate determination of the hydrodynamic blanket response requires numerical integration of the heat-transfer and fluid-mechanics equations. However, an approximate solution can be obtained analytically that models the essential features of the wave phenomena. 


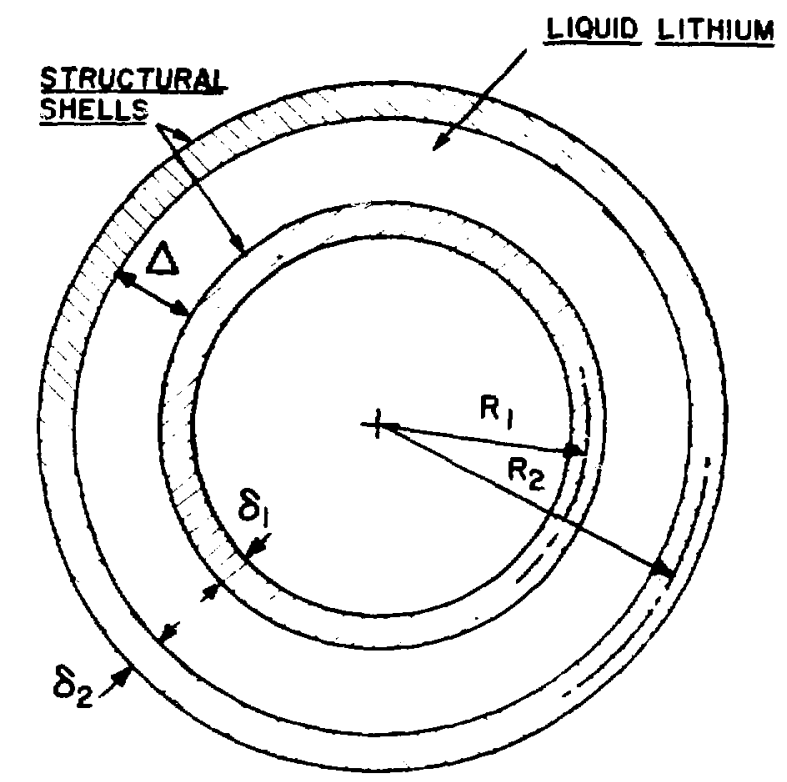

Fig. 34. Spherical ICF ieactor vessel model.

Because the nonuniform errergy deposition in the reactor blanket is volumetric, it induces a nonuniform temperature distribution, which resuits in an instantaneous pressure imhalance that is resolved with wave mrition. Dir model for its analysis is a liquid region between two immovable walls located at $r=R_{i}$ as shown in Fig. 34 .

The instantaneous temperature fistribution following neutron energy deposition induces an initial prussure perturbation, which may be approximited with an exporential profile $p(r)$,

$$
p=p_{1} e^{-\left[\left(r-R_{1}\right) / \lambda\right]}
$$

where $\lambda$ is the e-folding distance of the exponential energy reposition profile $(\lambda \sim 70 \mathrm{~cm}$ for lithium) and $p_{1}$ is the pressure generated at $r=R_{1}$,

$$
p_{1}=\frac{\beta b}{C_{p}^{0}} \frac{(1-f-x) Y}{\pi\left(R_{1}+R_{2}\right)^{2} \lambda} \text {. }
$$

In Eq. (69), $B$ is the adiabatic bulk modilus, b is the volume coefficient of thermal expansion, $c_{p}$ is the heat capacity, 0 is the density of the liquid medium, $Y$ is the fuel-pellet energy yield, $f$ is the fraction of yield in debris ions, and $x$ is the fraction of yield in $x$ rays. The derivation of these expressions is straightforward. Some details are in Sec. $v$ and Ref. 47.

The motion of the liquid blank $i$ medium resulting from the initial pressure imbalance is governed by the following set of linearized equations.

$$
\frac{\partial p}{\partial t}=a_{0}^{2} o_{0} \frac{\partial u}{\partial r}
$$

and

$$
\frac{\partial u}{\partial t}=\frac{1}{o_{u}} \frac{\partial p}{\partial r}
$$

where $L$ is the fluid velocity, $\varepsilon_{n}$ is the constant iiquid density, and $a_{0}$ is the sound speed; the initial conditions at $t=0$ are given by Eq. (68) and by $u(0)=0$ and the boundary conoitions by $u\left(R_{i}, t\right)=0$. The general solution of Eq. (70) may be written in terms of outward and inward propagai ing waves as follows.

$$
p=a_{0} o_{0}\left[f_{1}\left(r-a_{0} t\right)-f_{2}\left(r+a_{0} t\right)\right]
$$

and

$$
u=f_{1}\left(r-a_{0} t\right)+f_{2}\left(r+a_{0} t\right) \text {. }
$$

where the arbitrary functions $f_{i}$ are determineo from initial and boundary conditions.

Using the standard fourier series expansion, the solution is

$$
f_{1}=\frac{1}{2} \sum_{n=0}^{\infty} b_{n} \sin \left\{\frac{m}{\Delta}\left(r-a_{0} t\right)-\pi\left(\frac{1}{2}+n \frac{{ }^{R}}{\Delta}\right)\right\}
$$

and

$f_{2}=\frac{1}{2} \sum_{n=0}^{\infty} b_{n} \sin \left\{\frac{m}{\Delta}\left(r+a_{0} t\right)+\pi\left(\frac{1}{2}-n \frac{R}{\Delta}\right)\right\} ;$

here $\Delta$ is the blanket thickness, $\Delta=R_{2}$ - $R_{1}$. Because of Eq. (7l), the expression for the pressure perturbation can be written as

$\frac{p}{a_{0} o_{0}}=-\sum_{n=0}^{\infty} b_{n} \operatorname{ccs}\left[n \pi \frac{r-R_{1}}{\Delta}\right] \sin \left[\pi\left(\frac{l}{2}+\frac{n a_{0} t}{\Delta}\right)\right]$,

where the Fourier coefficients $b_{n}$ are 


$$
b_{0}=-\frac{\beta b}{a_{0} o_{0}^{2} c_{p}} \frac{(1-f-x) \gamma}{\pi\left(R_{1}+R_{2}\right)^{2} \Delta}\left(1-e^{-\Delta / \lambda}\right)
$$

and

$$
\begin{aligned}
b_{n} & =-\frac{2 \beta b}{a_{0} o_{0} c_{p}} \frac{(1-f-x) Y}{\pi\left(R_{1}+R_{2}\right)^{2} \lambda} \frac{\Delta / \lambda}{(\Delta / \lambda)^{2}+(n \pi)^{2}} \\
& x\left[1-(-1)^{n} e^{-\Delta / \lambda}\right], n \geq 1 .
\end{aligned}
$$

Several observations can be made based on this solution. First, the leading constant term $b_{0}$ gives the pressure rise that would obtain if the energy were added so slowly that waves did not develop. This agrees with the result obtained independently in Sec. $V$. Second, the first two terms describe the motion with less than $10 \%$ error; this is illustrated in Fig. 35, which shows approximations to the initial pressure distribution wotained by evaluating the series in Eq. (73) at $\mathrm{t}=0$ with $1,2,3$, and 4 terms successively.

Because the first harmonic component characterizes the motion with sufficient accuracy for cur purposes, we will examine the variation of its amplitude $b_{1}$ in some detail. Figure 36 illustrates the dependence of tinis amplitude on the blanket thickness $\Delta$, normalized with the e-folding scale $\lambda$. The current conceptual ICF

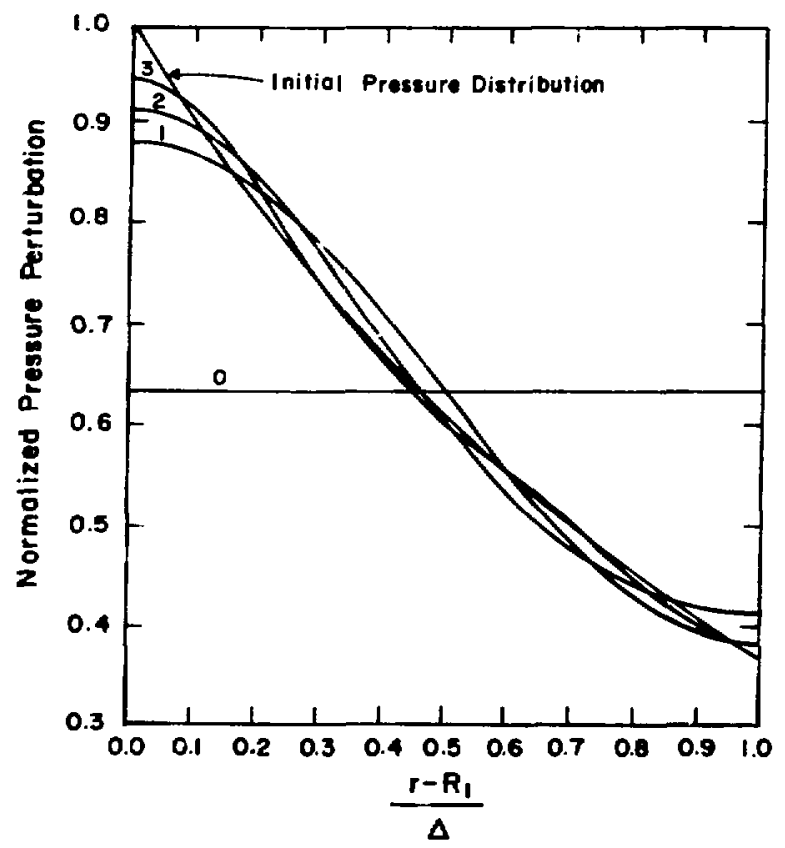

Fig. 35. Successive approximations to initial pressure distribution in liquid lithium blanket.

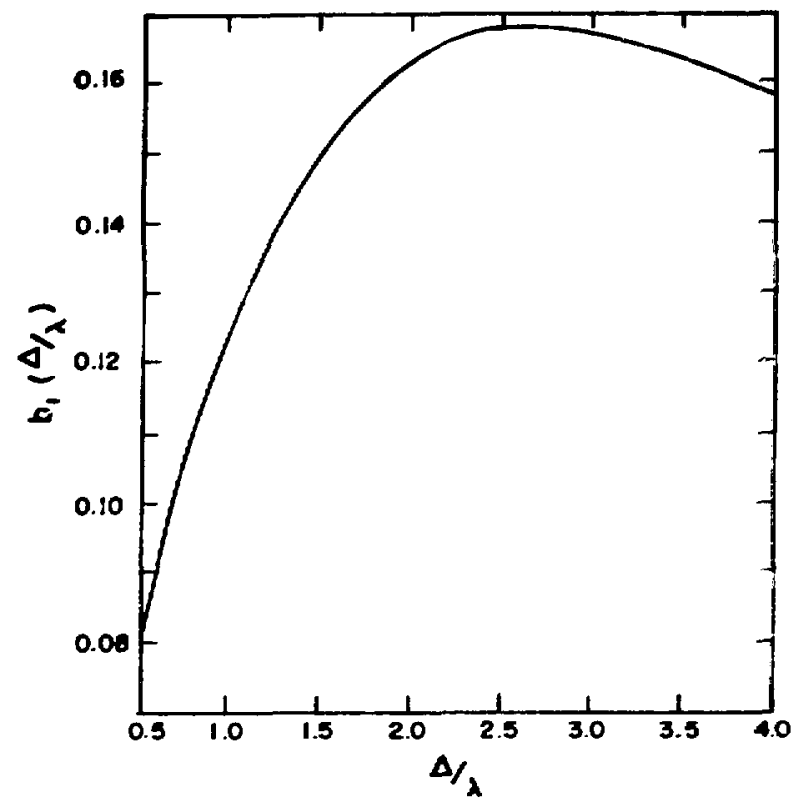

Fig. 36. Amplitude of first hamonic comoonent in liquid lithium blanket.

reactor designs have values of $\Delta \lambda$ in the range between 1.0 and 1.5 . Therefore, the amplitude of the pressure wave for these designs will increase steeply and approximately linearly with blanket thickness. This result indicates the incentive to design biankets that are as thin as possible, consistent with the needs of energy extraction. A more convenient form of this result is presented in Fig. 37, showing the ratio of sise ampiitude $b_{1}$ to the mean pressure $r$ ise $b_{0}$. Note that this ratio also increases with blanket thickness.

The impulse celivered by the first harmonit component to the inner shell is calculated easily by integrating the pressure at the wall, $r=R_{z}$. over the compression phase of the wave. The result is

$$
I_{1}=\frac{\Delta_{0}}{2 a_{0}}\left(\frac{1}{2}+\frac{1}{\pi} \frac{b_{1}}{b_{0}}\right),
$$

showing that the impuise is proportional to the blanket thickness $\Delta$ and to tie mean pressure rise $b_{0}$ and increases as the ratio $b_{1} / b_{0}$ increases. Substitution of explicit expressions for $b_{c}$ and $b_{1}$ shows that the impuise is proportional to the inverse square of the acoustic impedance $a_{0} 0_{0}$. 


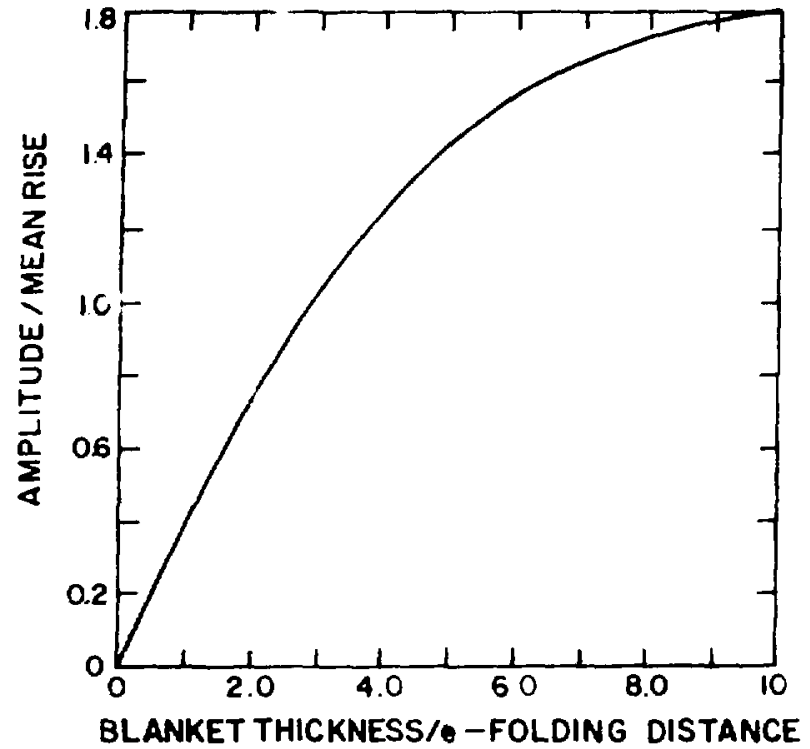

Fig. 37. Relative amplitude of first harmonic component of pressure wave in the blanket.

\section{Ir itium Breeding}

A fusion economy must be self-sufficient in tritium; that is, for each fusion reaction, at least one atom of tritium must be produced by nuclear transmutation of lithium. There are two tritium-breeding reactions of interest: ${ }^{7} L i\left(n, n^{\circ} \alpha\right)-2.46 \mathrm{MeV}, \quad$ a fast-neutron reaction, and ${ }^{6} \operatorname{Li}(n, a) T+4.79 \mathrm{MeV}$, which has a large thermal-neutron cross section. In addition, lithium is a relatively good neutron moderator. has good heat..transfer properties, and is reasonably abundant.

The ratio of tritium atoms produced to tritium atoms burned is called the tritiumbreeding ratio. Because only one neutron is produced in each $D-T$ fusion reaction, blankets must be designed for good neutron utilization. This requirement demands occasional use of neutron multiplying and moderating materials. The most important sources of neutrons in blanket regions are $(n, 2 n)$ reactions in structural and other materials such as beryllium and sodium that may be included for this purpose. Tritium production from ${ }^{7} L$ also uses nevi-ons economically because each nuclear reaction results in the birth of a neutron that can be reacted with $6_{L i}$. Tritiumbreeding ratios in the range 1.1 to 1.5 can be obtained in blankets consisting of structural materials and natural lithium with thickresses less than $1 \mathrm{~m}$. Breeding ratios exceeding 2 can ve obtained in blankets rontaining lithium enriched in ${ }^{6} L$, combined with a neutron muitising and moderating material such as beryllium.

significant increases in iotal entrgy released per fusion reaction can ve oblaineo by including materlals that undergo pxoergic ne"itron reactions. Tritium production by neutron capture in ${ }^{6} L i$ resuits in a net release of energy, so that total energy release per fusion reaction generally increases with increases in ureeding ratio. Other nuclear reactions that amplify fusion energy include $(r, y)$ reactions in siructural materials.

\section{E. Materials ffiects}

1. General Considerations. Possiole blanket structural materials include such refractory metals as niobiui, molybdenumi, vanadum, anc alloys of these materials. Stainless steels and sintered aluminum products also may be used. Considerations important in determining final cnoices include temperature limitations, corrosion resistance, fatigue strength, radiation damegc effects. neutron-inauced radioactivity and afterieat, ano availability. Niobium is attractive because of its compatibility with lithium at high temperatures and its decirable neutronics characteristics. However, it may not be sufficiently abundant to satisfy requirements, and it has some long-lived neutron-incuced radioactuve isotopes. Nolyboenum, which is abundant in the continental 115, also is an attractive blanket structural material. it is compatible with lithium, has good migh-temperature mechanical properties, is relatively impermeasle to the tiffusion of hyarogen isotopes, and has a large $(n, 2 n)$ cross section for high-energy neutrons. Some engineers are reluctant to use molybdenum for this application because of the difficulty of preventing brittle weld zones. However, significant progress has been made in the development of techniques for fabricating molybdenum and molybdenum alloys, and it may prove to be the preferred structural material for hightemperature reactor blanket and primary-loop heat exchanger systems. Two materials, vanadium and sintered aluminum products, offer significant 
ddvintades with respect to neutron-induced radioactivity. It is not known whether vanadium for vanadium alioysj has sufticient strength and lithium-corrosion resistance for use in 'ser fusion applications. Sintered aluminum products and stainless steels are limited to operation below $2790 \mathrm{k}$ because of lithium corrosion and serength limidions. Corrosion of stainless steei d nigh temperatures might be dlleviated by retractory-metal cladding.

2. Danage. The extent lo which structural materials are damaged by the products of fusion redctions is largely unknown, but it will be very important in determining material choices and component lifetimes. large amounts of protium and iritium will be produced in the structural materials and lithium coolant. The formation of nyorides and the resulting embrittlement could be a serious structural problem. Niobium and vanadium form stable hydrides at low temperatures, but hydrogen solubllity in these materials decreases rapidly with increasing lemperature. If reactor cooldowns can be programmed to allow hydrogen to diffuse out of these materials before ambient temperatures are reached, the hydrogen embrittlement problems may not be severe. Molybdenum does not form hydrides and has $a$ very low hydrogen solutility.

Neutron primary damage is most severe for the wall surrounding the central cavity. For niobium, the often-specified first-wall material, Fig. 38 (Ref. 48) shows the number of atomic displacements per atom, the amount of niobium destroyed by transmutations ( $98 \%$ of which is transmuted to $z$ ir conium), and the amounts of hydrogen and helium produced in one year of operation with a pulse rate of one 100-MJ microexplosion per second--all plotted versus cavity diameter.

Some neutron energy is deposited directly in structural components, and, in addition, $(n, r)$ reactions occur thrcughout the reactor system. The gamma-ray energy is deposited primarily in high-density structural regions. Energy deposition in structural components results in thermal gradients that, in tui.., give rise to thermal expansion and thermal stresses. Also, temperature gradients are superimposed in the blanket because of coolant flow. Hodeling and analysis of thermal and mechanical stresses are discussed in Sec. $V$.

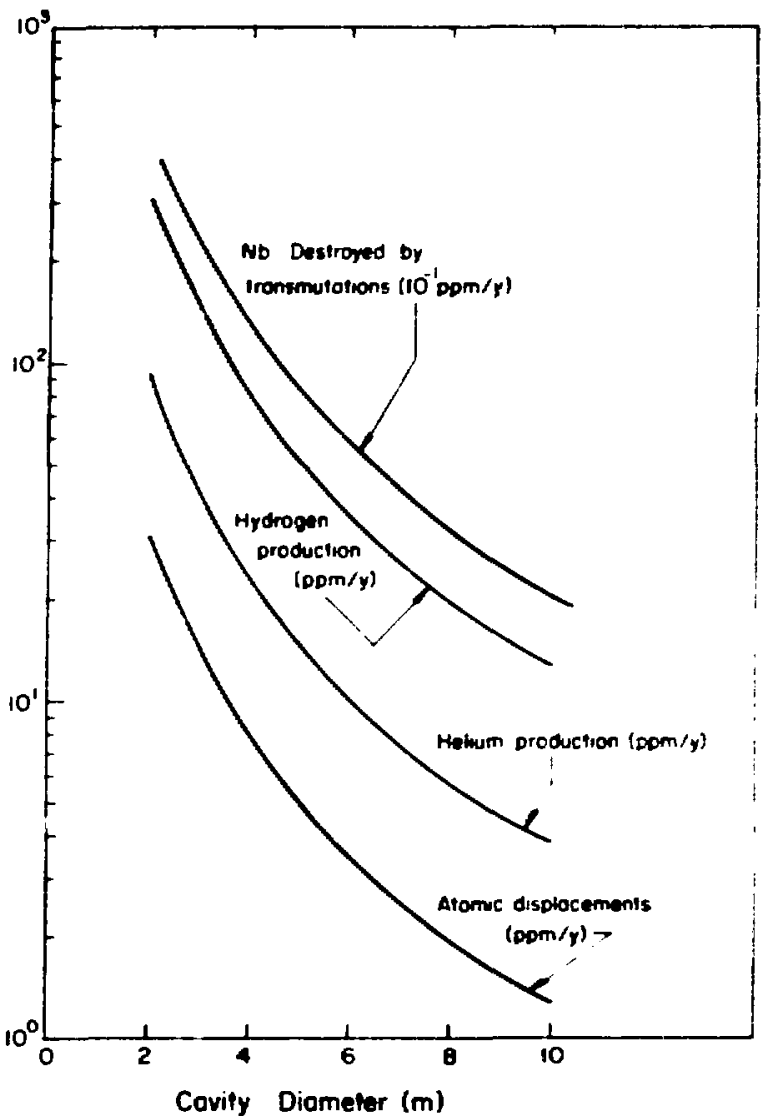

Fig. 38. Effect of cavity diameter on niobium transmutations to $\mathrm{H}_{2}$ and the in reactor cavity wall (for 1 year of coeration with one 100-MJ fusion-pellet microexplosion per second).

3. Activation and Afterheat. The activity induced in reactor structural materials by neutron irradiation poses a threat to workers and necessitates remote operations. Assessing requirements for remotely operated maintenance and handling equipment requires estimates of these induced activities. Estimates of activity in waste material resulting from first-wall change out and the replacement of other structural parts that resuit in waste depend on such activation estimates. In this connection, it has been shown that induced activity and afterheat in a fusion reactor are relatively small compared to those in fission reactors.

\section{F. Parametric Studies}

Preliminary and scoping analyses of ICF reactor concepts require parametric studies of the 
neutronic effects to assess sensitivity to variations in neutron spectra and other parameters. Unfortunately, in general, neutronic calculations are laborious and time-consuming. The computational labor needed to perform some parametric studies (for example, to investigate effects of different pellet neutron spectra on blanket response) may be reduced significantly by exploiting the properties of the adjoint of the neutron transport operator. The approach is outlined below.

Let us represent the neutron transport equation in symbolic form

$$
L \psi=S,
$$

where $L$ is the transport operator, $\psi$ is the neutron flux, and $S$ is the source.

In general, we seldom are interested in the detailed behavior of the solution but only in the responses to the neutron flux $\psi$ that $c$ an be characterized with a single number, such as the tritium-breeding ratio or the blanket heat production. These responses are represented symbolically as functionals $f$ of $\omega$ given by

$$
f=(U, R),
$$

where $R$ is the appropriate response function and the parentheses indicate the inner product, that is, the integral of the product over the appropriate region.

From the theory of differential operators, we know that $L$ has an adjoint $L$ * such that

$$
\left(L \psi, w^{\star}\right)=\left(\psi, L \star \psi^{\star}\right)
$$

for any function $\psi^{*}$. Let $\omega^{*}$ be the solution of the adjoint equation with the source given by the response furiction $R$; that is,

$$
L \star U^{\star}=R
$$

Then, using Eqs. (77) and (80) in Eq. (79), we obtain the equality

$$
\left(S, \omega^{*}\right)=(\omega, R)
$$

which, because of Eq. (78), implies that

$$
f=\left(S, \psi^{\star}\right)
$$

Thus, the desired response $f$ can be calculated for any given neutron source $S$ from the solution w of the adjoint Eq. (80).

The advantage of Eq. (81) over Eq. (78) is that it enables us to determine responses to any number of sources with only one solution w* of the adjoint transport Eq. (80). Therefore, once the adjoint solution has been calculated, the effects of a varying pellet spectrum $S$ can be investigated parametrically with very little effort; the additional computations require only evaluations of definite integrals indicated by Eq. (81).

We illustrate this advantage with two examples. Figure 39 shows the neutron spectrum corresponding to a pellet composed of $1 \mathrm{mg}$ of pure, equimolar D-T compressed uniformly to a radius at which $O R=1 \mathrm{~g} / \mathrm{cm}^{2}$. The spectrum was calculated with the LOS Alamos National Laboratory discrete-ordinates code ONETRAN-DA in $S_{8} P_{3}$ approximation and required a few seconds of $\operatorname{CDC} 7600$ computer time. The calculation used 30

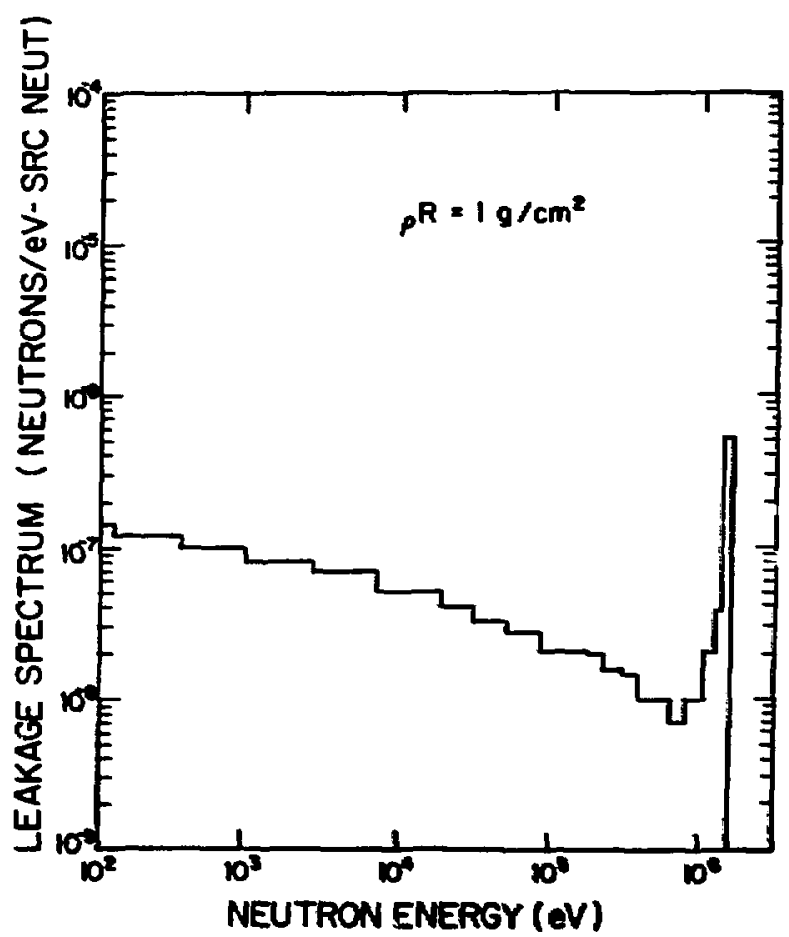

Fig. 39. Peilet ieakage spectrum. 
group cross sections processed from the nuclear data file ENDF/B-IV with the LOS Alamos processing code system NJOY. The spectrum is normalized to one D-T source neutron and contains three regions: a 14.1-MeV fision peak, a region of first- and second-collided flux, and $a$ Because of the presence of $(n, 2 n)$ reactions, the area under the histogram is slightly larger than unity. Spectra for pellets of $O R=2$ and $4 \mathrm{~g} / \mathrm{cm}^{2}$ were similarly calculated. Each spectrum represents a source term $S$, which is to be folded with the adjoint function $w^{\star}$.

We first illustrate the rapid calculation of tritium-breeding ratio for pellets of varying OR. In this case, the adjoint source is the response function for tritium production with the cross sections for natural lithium. The energydependent cross sections are multiplied by the lithium atom density in a typical commercial reactor blanket. Solutions of the adjoint to the transport equation give the adjoint function for tritium production, shown in Fig. 40. The extreme right-hand side of the plot shows the value of the tritium-breeding ratio expected for a pure 14.1-MeV source, approximately 1.35; this value agrees with the direct determination using Eq. (77) with a 14.1-Mev source. However, the adjoint function contains implicitly the tritiumbreeding ratio for all possible pellets. In particular, the tritium-breeding ratio for pellets of $O R=1,2$, and $4 \mathrm{~g} / \mathrm{cm}^{2}$ was determined by folding the adjoint function shown in Fig. 40 with the pellet spectra, such as Fig. 39, in a simple desk calculator operation. The result is shown in Fig. 41. Note that for very high values of of

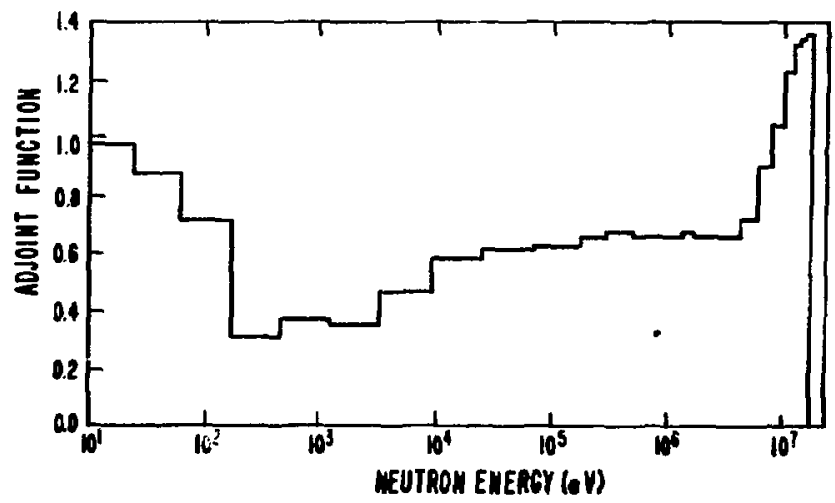

Fig. 40. Adjoint function for tritium production.

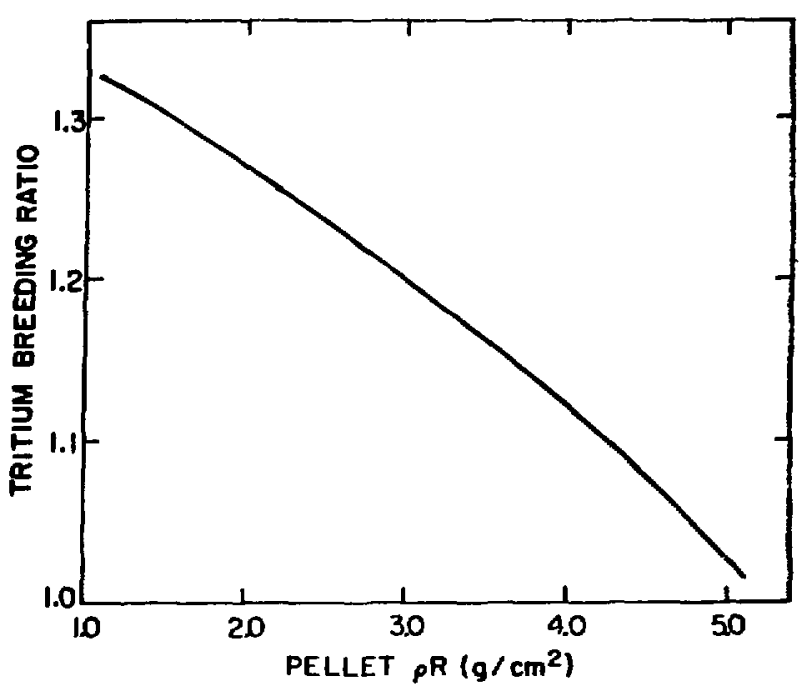

Fig. 41. Tritium production as a function of pellet oR.

the tritium-breeding ratio falls to near unity. Generating the same figure by direct calculations of the tritium-breeding ratio from Eqs. (77) and (78) would have required several solutions of the transport Eq. (77).

The adjoint function also can be used in tailoring pellet spectra to produce a given blanket response or to mitigate an undesirable response. The method is illustrated by showing how helium production in the first wal of the vessel can be minimized by use of nigh-oR pel-lets. Here the energy-dependent helium production in niobium is multiplied by the niobium atom density to obtain the response function (that is, the source for the adjoint equation). The adjoint function plotted in Fig. 42 shows a sharp threshold; pellet spectra degraded below this threshoid should give sharply reduced helium production. The first-wall helium production as a function of pellet oR shown in Fig. 43 confirms the behavior predicted by the adjoint function. However, the effect is not very dramatic: a four-fold increase in of (from 1 to 4) reduces the helium production by not much more than $60 \%$.

The examples demonstrate use of the adjoint function in reducing computational effort in investigating effects of pellet spectra on blanket response and in tailoring pellet spectra to achieve a desired blanket response. 


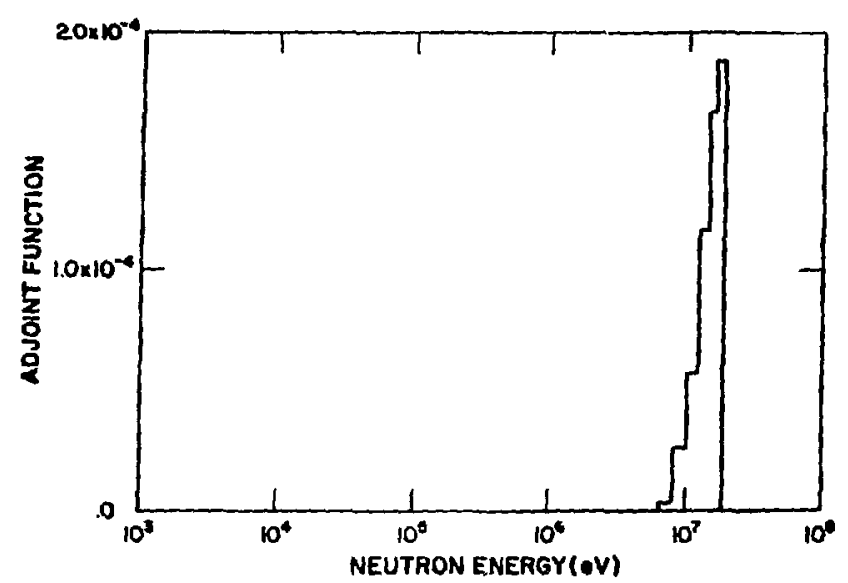

Fig. 42. Adjoint function for first-wall helium production.

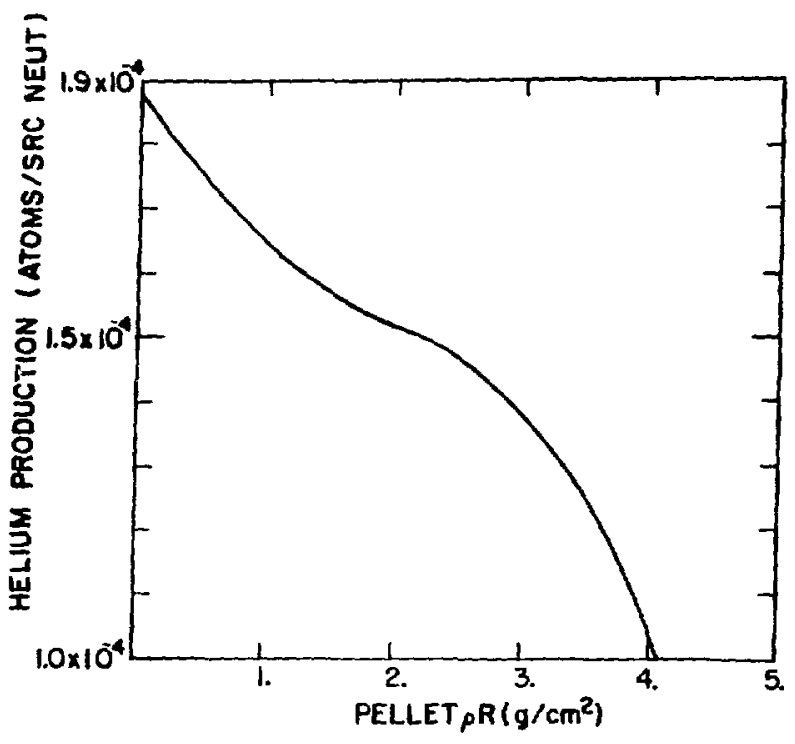

Fig. 43. First-wall helium production as a function of pellet oR.

\section{LOAD AND STRESS ANALYSIS}

\section{A. General}

At present, the characteristics of ICF pellet energy releases are not known sufficiently well to examine specific containment vessel designs. It is more appropriate to formulate the impulse containment problem in general and to derive results in the form of explicit expressions from which magnitude estimates and parametric dependencies (trends) can be inferred conveniently and rapidly. To carry out this task, we use assumptions and approximations as required to perform the analys is.

The general ICF impulse containment problem may be formulated in the following way. For a total pellet energy release $Y$, the energy to be contained is $(f+x) y$ where $(f+x)$ is the frac$t$ ion of energy yield in debris and $x$ rays f that is, $1.0-f-x$ is the fraction of energy release that escapes the containment vessel as high-energy neutrons). Assuming that the response of the containment vessel remains in the elastic regime (necessary for long-term repetitive operation) and that its wall thickness $\delta_{j}$ is small in comparison to the radius $R_{i}$, the elastic energy stored in the volume of the containment vessel material as two-dimensional membrane strain is given by $\left(4 \pi R_{j}^{2} \delta_{i}\right) \cdot\left[E E^{2} / 2(1-v)\right] \cdot 2$ where $E$ is Young's modulus, $\varepsilon$ is the linear strain, and $v$ is Poisson's ratio. It is reasonable to postulate that there exists a functional relation between the energy contained in the cavity and the energy stored elastically in the wall material.

$$
2\left(4 \pi R_{i}^{2} \delta_{i}\right) \frac{E E^{2}}{2(]-v)}=F[(f+x) \gamma] .
$$

Our objective is to derive the dependence $F$ and thus to obtain explicit expressions relating energy yield $Y$ to containment vessel parameters.

The function, $F$, depends on the mechanism of energy transfer from the cavity medium into the wall material. It is clear that $F$, in general, is not an identity function because it is not possible to transfer the entire energy content of the cavity into the wa11. This circumstance is analogous and related to the fact that in ICF only a fraction (5 to 50\%) of the pusher $k$ inetic energy can be transferred to the thermonuclear fuel. To determine $F$, it is necessary to investigate a specific containment model and momentum coupling between the energy release and the vessel wall.

\section{B. The Model}

The containment vessel model most suitabie for obtaining explicit results consists of two concentric spherical shells with a layer (blanket) of liquid between them as shown in Fig. 34. To 
proceed with the analys is we make the following as sumptions.

- Structural shells are spherical.

- Shell thickness $\delta_{i}$ is small relative to the shell radius $R_{i}$.

- Blanket thickness $\Delta$ is small relative to the radius.

- Structural shell responses remain in the elastic regime.

- Load pulses are short relative to the period of free (elastic) shell vibrations.

The last assumption permits us to approximate with Dirac delta functions loads applied to the vessel wall. It is a very good approximation because load pulse durations in ICF are estimated to be in the microsecond range, whereas periods of free shell vibrations are in the millisecond range for radii exceeding $1 \mathrm{~m}^{47}$ The use of thinshell approximations in stress calculations for the containment vessel walls is justified because in practical (commercial) applications these walls will be thin relative to the vessel radius $\left(\delta_{j} / R_{i}<0.01\right)$ to avoid excessive neutron energy deposition and material cost.

To obtain conservative estimates that are valid when voids or bubbles develop in the liquid blanket or when the blanket is absent, we omit the hydrodynamic coupling term in the equation governing the elastic response of a thin shell to the impulse per unit area I and obtain the following formulation. 47

$$
0 \frac{d^{2} w_{i}}{d t^{2}}+\frac{2 E}{(1-v) R_{i}^{2}} w_{i}=0 \text {, }
$$

where $w_{i}$ is the radial shell displacement related to the tensile or compressive strain $E$ by the geometric compatibility relation

$$
\varepsilon_{i}=w_{i} / R_{i}
$$

and $t$ is the time elapsed aiter impulse application.

The initial conditions for the solution $w_{i}(t)$ are given by

$$
w_{i}(0)=0 \text { and } \frac{d w_{i}(0)}{d t}=\frac{I}{0 \delta_{i}} \text {. }
$$

The effect of hydrodynamic coupling of the shell to the liquid blanket will be discussed later.

The solution of Eq. (83) that satisfies compatibility condition Eq. (84) and initial condi$t$ ions Eq. (85) is

$$
\varepsilon_{i}=\sqrt{\frac{T-\nu}{2 E D}} \frac{I}{\delta_{i}} \sin \sqrt{\frac{2 E}{(1-v) R_{i}^{2} 0}} t ;
$$

therefore the maximum strain is

$$
\varepsilon_{i m}=\sqrt{\frac{1-v}{2 E D}} \frac{I}{\delta_{i}} .
$$

Using Eq. (87) and Eq. (82), we obtain an expression for the dependence of $F$ on the impulse applied to the wall,

$$
F=2 \pi R_{j}^{2} \frac{I^{2}}{D \delta_{i}}
$$

To complete the analysis, we examine different mechanisms by which the energy released in a pellet microexplosion generates an impulse at the vessel wall, and we derive the corresponding expressions for $I$ in terms of $Y$.

\section{Impulse Generation}

Thermonuclear burn of $D-T$ fuel in an ICF fuel pellet releases energetic (14-MeV) neutrons, $x$ rays, and energetic ionized pellet debris. These energy forms apply impulse to the wall through evaporation recoil, debris impact, blast wave reflection, and blanket thermal expansion.

In addition to the above mechanisms, excitation of stress waves in the vessel wall generates stresses different from membrane stresses; we will analyze them in a separate section.

1. Evaporation Recoil. The pulse of $x$ rays produced in a fusion microexplosion is absorbed in a thin layer of wall material, part of which may evaporate and thus generate an impulse at the wall. The magnitude of the recoil impulse per unit area $I_{r}$ maximized with respect to the mass of material evaporated is given by 47 


$$
l_{r}=\frac{Y \times \eta}{4 \pi R_{1}^{2} \sqrt{2 H}}
$$

where $H$ is the heat of vaporization of the wall material and $n$ is the effectiveness coefficient that accounts for the fact that not all vapor moves away from the wall with maximum attainable velocity; for example, for a particular model, namely that of a Riemann wave extending to vacuum, $n=0.15$.

With this expression for impulse, Eqs. (82) and (87) lesult in the following radius-yield relation.

$$
R_{i}=\left[\frac{E}{\pi-v) H_{0}}\right]^{1 / 4} \sqrt{\frac{\gamma x}{8 \pi \sigma_{m} \delta_{i}}} n \text {, }
$$

in which the strain $\varepsilon_{i m}$ has been eliminated in favor of maximum allowable stress $\sigma_{m}$ using

$$
\sigma=\frac{E \varepsilon}{T-v} .
$$

2. Impact of Pellet Debris. The kinetic energy of the high-velocity fuel pellet debris delivers to the vessel wall an impulse per unit area, $I_{d}$, whose magnitude is 47

$$
I_{d}=\frac{\sqrt{Y f M}}{2 \sqrt{2} \pi R_{1}^{c}}
$$

Here $M$ is the fuel-pellet mass. Equation (92) is conservative because it is based on the assumption that all kinetic energy is converted into impulse. In practice, part of the kinetic energy will appear as heat and will produce a recoil impulse whose magnitude can be estimated from Eq. (89) with an appropriate value for the energy fraction $x$. The two recoil impulses cannot be combined because, in general, they occur at different $t$ imes.

In this case, the radius increases as the fourth root of the energy yield.

$$
R_{i}=\frac{1}{2} \sqrt{\frac{1}{\pi \sigma_{m} \delta_{i}}}\left[\frac{\text { EMYf }}{(1-v) 0}\right]^{1 / 4} \text {. }
$$

3. Blast Wave Reflection. When the ambient density in the cavity exceeds about $10^{14}$ atoms $/ \mathrm{cm}^{3}$, the pellet microexplosion will generate a spherical blast wave. The impulse experienced by the reactor vessel wall during blast wave reflection is easily estimated as the product of the pressure at the wall behind the reflected wave, Eq. (7) of Ref. 47, and the pulse duration, which we approximate with the transit time of a sound wave through the shock compressed layer of the ambient cavity gas. The resulting expression for the impulse per unit area $I_{b}$ is

$$
I_{b}=\sqrt{\frac{3 \gamma-I}{8 \pi}} \sqrt{\frac{Y \sigma_{0}}{R_{i}}}
$$

where $o_{0}$ is the ambient mass density of the cavity medium and $r$ is the constant ratio of specific heats in that medium. In the derivation of Eq. (94), the Taylor-Sedov similarity description $^{18}$ of the blast wave was used. The validity of this solution deteriorates as the pellet mass increases and approaches the mass of the ambient cavity medium; at that point, a modified blast wave theory ${ }^{19}$ should be used to obtain accurate results. Unfortunately, any analys is of the blast phenomena that is more complex than the TaylorSedor description precludes obtaining an analytic impulse estimate analogous to Eq. (94).

When the above assumptions are valid, the radius is directly proportional to the yield with the proportionality constant depending on the ambient cavity density $o_{0}$,

$$
R_{i}=\frac{1}{16 \pi} \frac{3 y-1}{1-\nu} \frac{E o_{0}}{0 \sigma_{m}^{2} \delta_{i}^{2}} y_{f} .
$$

4. Thermal Response of the Blanket. Lithium blankets, both liquid and solid compounds in pellet form, are designed to convert neutron kinetic energy into thermal energy and will expand during reactor operation. The mean pressure increase caused by confinement of a liquid lithium blanket is 47

$$
p=\frac{\beta b}{C_{\ell} D_{l}} \frac{Y(l-f-x)}{V} \text {, }
$$

where $p$ is the pressure increase, $B$ is the adiabatic bulk modulus, $b$ is the volume coefficient of thermal expansion, $c_{l}$ is the heat capacity of liquid lithium, $o_{\ell}$ is the density of Biquid 
lithium, and $v$ is the blanket volume. This estimate is based on the assumption that neutron energy deposition is sufficiently slow or uniform and does not induce dynamic imbalances in the process. Actually, neutron energy is deposited with an exponentially decreasing intensity in a time that is short relative to the hydrodynamic response time and therefore generates pressure waves in the liquid blanket. To analyze these waves and to model their effect, we solved the acoustic equations (in the plane wave approximation) for pressure $p$ and velocity $u$, perturbations in a liquid medium between two concentric shells shown in Fig. 34. The medium was initially at rest with an exponentially decreasing (from the inner shell $R_{1}$ to the outer shell $R_{2}$ ) pressure distribution induced by a postulated instantaneous neutron energy deposition with the scale depth $\lambda$ ( $\lambda \approx 70 \mathrm{~cm}$ for liquid lithium). The details of the solution and the neutronic analysis are presented in Sec. IV, together with the neutronic analysis. Here we recapitulate the conclusions relevant to the present discussion.

- The mean pressure $r$ ise and the first harmonic component account for nearly $90 \%$ of the deposited energy and therefore provide an approximate description of the phenomena that is adequate for the purpose of this report.

- The ratio of the amplitude of the first harmonic to the mean pressure rise increases nearly linearly with the nondimensional blanket thickness $\Delta / \lambda$ for values of $\Delta / \lambda<4$, as shown in $F$ ig. 37. At the typical value of the blanket thickness, $\Delta / \lambda \approx 1.6$, that ratio is approximately 0.50 . Therefore, in stress calculations the mean pressure estimate given by Eq. (96) should be mul:iplied by a factor of 1.50 to account for the transient overpressure.

The analysis presented in Sec. IV also verified the intuitively expected fact that the pulse duration to be used with Eq. (96) to estimate the impulse at the wall is given by $\tau=\Delta / a_{\ell}$, where $a_{\ell}$ is the sound speed in the blanket liquid given by $a_{\ell}=\sqrt{B / a_{\ell}}$. Using these results, the expression for the impulse delivered to the vessel wall because of confining the thermal expansion of the blanket becomes

$$
I_{t}=\frac{1.5 a_{\ell} b}{4 \pi c_{\ell} R_{i}^{2}} Y(1-f-x)
$$

$I_{t}$ is independent of the blanket thickness $\Delta$ because of the approximation $v \approx 4 \pi R_{i}^{2} \Delta$.

Using this expression for the impulse with Eqs. (82) and (87) results in the following radius-yield relation,

$$
R=\left[\frac{E \beta}{2(T-V) O 0_{\ell}}\right]^{1 / 4} \sqrt{\frac{T .5 b}{4 \pi \delta_{i} C_{\ell} \sigma_{m}} Y(1-f-x)} .
$$

\section{Elastic Stability}

The pressure increase in the blanket not only generates tensile and compressive stresses in the structural shells, but also tends to buckle the inner shell. Assuming that the critical value of the pressure for this mode of failure is the same as that developed for a static load application and substituting an expression for $i t^{49}$ into Eq. (96), we find that the radius of the vessel cancels out and the wall thickness, $\delta_{1}$, required to prevent buckling, increases as the square root of the yield.

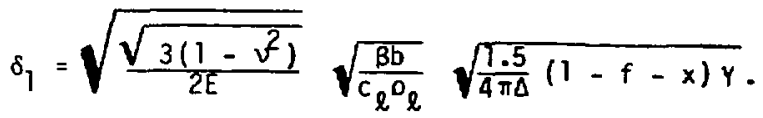

For a cylindrical vessel the corresponding formula is

$$
\delta_{1}=\left(1-v^{2}\right)^{3 / 10}\left[\left(\frac{\beta}{C_{\ell} D_{\ell}}\right) \frac{1.5 L Y(1-f-x)}{0.807 E V}\right]^{2 / 5} R_{J}^{3 / 5} .
$$

Here $V$ is again the blanket volume and $L$ is the unsupported cylinder length. Equation (100) depends on the cylinder radius $R_{j}$, but the dependence is weak; for example, when the blanket volume corresponding to the length $L$ is approximated with $V \approx 2 \pi R^{L \Delta}$, then $\delta_{1}$ 
Equations (99) and (100) are applicable only when the vessel design does not provide for the relief of thermally induced blanket pressure increase and when it is impractical or undesirable to provide stiffening ribs or flanges.

\section{E. Sample Results}

The radius-yield relations developed above are evaluated in the range of parameters applicable to ICF, and the results are presented in Fig. 44. The constants used in the evaluation are listed in Table I.

In these calculations, the shell thickness is $\delta_{i}=1 \mathrm{~cm}$, the pellet mass is $M=0.5 \mathrm{~g}$, and the ambient density of the cavity medium is $0_{0}$ $=1.5 \times 10^{-7} \mathrm{~g} / \mathrm{cm}^{3}$, which corresponds to approximately 0.1 torr of argon at $500 \mathrm{~K}$. This density apparently is the highest that may allow satisfactory propagation of the laser beam through the cavity. We see that the structural strength requirements for an unprotected containment vessel wall are dominated by evaporation recoil and elastic buckling and that the blast wave effects are negligible.

The magnitudes of the results show that the structural requirements of ICF impulse containment are mild; the radij required are less than $1 \mathrm{~m}$.

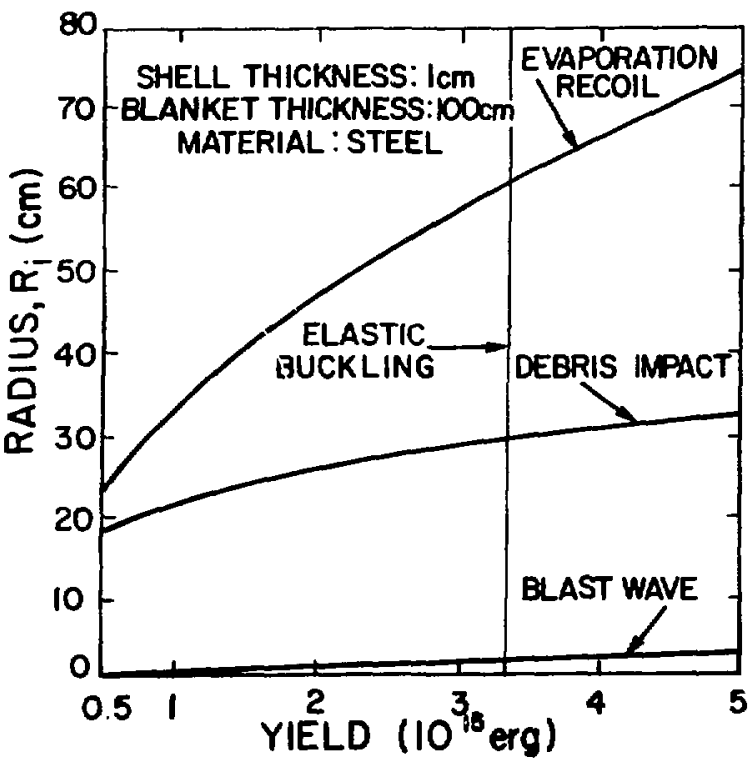

Fig. 44. Radius-yield relations for ICF reactor vessels determined by structural strength requirements.
TABLE I

REPRESENTATIVE PARAMETER VALUES

\begin{tabular}{|c|c|}
\hline Quantity & Value \\
\hline$\alpha$ & $14.4 \times 10^{-6} \mathrm{~K}^{-1}$ \\
\hline b & $1.39 \times 10^{-4} \mathrm{~K}^{-1}$ \\
\hline B & $1.09 \times 10^{11} \mathrm{dg} / \mathrm{cm}^{2}$ \\
\hline$\Delta$ & $100 \mathrm{~cm}$ \\
\hline$c_{\ell}$ & $3.55 \times 10^{7} \mathrm{erg} / \mathrm{g} \mathrm{K}$ \\
\hline$E$ & $2 \times 10^{12} \mathrm{dym} / \mathrm{cm}^{2}$ \\
\hline$n$ & 0.25 \\
\hline $\boldsymbol{Y}$ & 1.20 \\
\hline$H$ & $7.47 \times 10^{10} \mathrm{erg} / \mathrm{g}$ \\
\hline o & $7.81 \mathrm{~g} / \mathrm{cm}^{3}$ \\
\hline$o_{\ell}$ & $0.52 \mathrm{~g} / \mathrm{cm}^{3}$ \\
\hline$a_{m}$ & $10^{9} \mathrm{dyn} / \mathrm{cm}^{2}$ \\
\hline$v$ & 0.30 \\
\hline$x$ & 0.05 \\
\hline$f$ & 0.20 \\
\hline
\end{tabular}

Therefore, the design of contaimment vessels will be determined not by the structural integrity constraints but by lifetime considerations that depend on first-wall material loss and neutron damage mechanisms.

F. Hydrodynamic Coupling

The natural oscillations of a structural shell described by Eq. (86) are modified significantly when the shell is surrounded by a blanket because of the transfer of $k$ inetic energy from the shell to the liquid. This energy-loss mechanism, which is much more effective than the internal damping in the shell material, will be estimated in this section.

In an unbounded fluid, the pressure pulses generated by an oscillating sphere carry the energy away in the form of sound waves. In a fluid blanket of finite thickness, the pulses are reflected at the outer shell, and the wave interaction pattern must be determined to obtain a complete description of the phenomena. Here we limit the analysis to a time before the first 
reflected wave returns to the inner shell. With this restriction, we explicitly model the coupling of the shell to the liquid blanket and show that the motion is overdamped.

The differential equation governing the elastic motion of a shell hydrodynamically coupled to the surrounding fluid is obtained by adding to Eq. (83) a term representing the pressure exerted by the fluid. When the fluid is inviscid and the propagation of spherical waves can be approximated with locally plane wave fronts, this term is $o_{\ell} a_{\ell}\left(d w_{j} / d t\right)$, where $a_{\ell}$ is the sound speed in the liquid and $o_{\ell} a_{\ell}$ is known as the acoustic impedance of the medium. With this addition, the differential equation for the radial shell displacement is

$$
0 \frac{d^{2} w_{i}}{d t^{2}}+\frac{o \ell^{a} \ell}{\delta_{i}} \frac{d w_{i}}{d t}+\frac{2 E}{R_{i}^{2}(1-v)} w_{i}=0,
$$

and the boundary conditions are given by Eq. (85) supplemented with the condition of no cavitation.

The solution of this system is

$$
w_{i}(t)=C_{i}\left(e^{-\omega_{1} t}-e^{-\omega_{2} t}\right)
$$

with the characteristic roots given by

$$
w_{1,2}=\frac{o \ell^{a} \ell}{20 \delta_{i}} \pm\left[\left(\frac{o \ell^{a} \ell}{20 \delta_{i}}\right)^{2}-\frac{2 E}{o R_{i}^{2}(1-v)}\right]^{1 / 2}
$$

and the constant $C_{j}$ by

$c_{i}=-\frac{1}{20 \delta_{i}}\left[\left(\frac{o \ell^{\mathrm{a}} \ell}{20 \delta_{i}}\right)^{2}-\frac{2 E}{o R_{i}^{2}(1-v)}\right]^{-1 / 2}$.

In ICF applications the inequality

$$
\left(\frac{o l^{a} \ell}{20 \delta_{i}}\right)^{2}>\frac{2 E}{R_{i}^{2}(1-v)}
$$

is usually satisfied ${ }^{47}$ and therefore Eq. (102) is exponential and not oscillatory.

Equation (102), although explicit, is too complex to allow convenient interpretation. To understand the physical meaning of different terms and thus to identify parameters that determine its behavior, we use Eq. (105); it implies that the isolated shell response represented by the terin $2 E / O R_{i}^{2}(1-v)$ may be neglected in comparison to the hydrodynamic coupling effect $\left(o_{\ell} a_{\ell}\right)^{2} /\left(20 \delta_{j}\right)^{2}$. except in $\omega_{2}$ where the ratio of these terms is the dominant contribution. With this approximation and an appropriate expansion of the square root, the result simpiifies to

$$
\begin{gathered}
\omega_{1} \approx \frac{o_{\ell} a_{l}}{o \delta_{1}}, \\
\omega_{2} \approx \frac{\delta_{i}}{R_{i}} \frac{2 E}{R_{i}(1-\nu) o_{\ell} a_{\ell}},
\end{gathered}
$$

and

$$
c_{i} \approx-\frac{1}{o_{\ell} a_{\ell}}
$$

These terms have physical interpretations: in is the ratio of the hydrodynamic impedance to the shell mass per unit area, $w_{2}$ is the ratio of the shell elastic stiffness to the hydrodynamic impedance modified by the aspect ratio $\left(\delta_{i} / R_{i}\right)$, and $c_{i}$ is the negative of the ratio of applied impulie to the hydrodynamic impedance.

To illustrate the behavior of the sheilblanket system, we evaluated EG. (102) for I $=183 \mathrm{dyn}-\mathrm{s} / \mathrm{cm}^{2}, \quad R_{i}=500 \mathrm{~cm}, \quad$ and $\delta_{i}$ $=1.35 \mathrm{~cm}$ (the values of the remaining parameters are listed in Table I) and plotted the resu't in Fig. 45. It shows that the time, $t_{m}$, given by

$$
t_{m}=\frac{\ln \left(\omega_{1} / \omega_{2}\right)}{\omega_{1}-\omega_{2}} \text {, }
$$

at which the strain (and stress) peaks occurs we]1 before the return of the first pressure wave given by $t_{r}=2 \mathrm{~N} \mathrm{a}_{\ell}$ and that the stress reaches its maximum value very rapidly in comparison to the subsequent relaxation. For the condjtions of this example, the maximum stress is $\sigma\left(t_{m}\right)$ $=4.17 \times 10^{6}$ dyne/cnf; this value is approximately 10 times lower than the corresponding value for an isolated shell given by Eq. (87). Such a result is physically obvious. The inertia of the liquid blanket inhibits shell expansion and thus reduces strain and stress. 


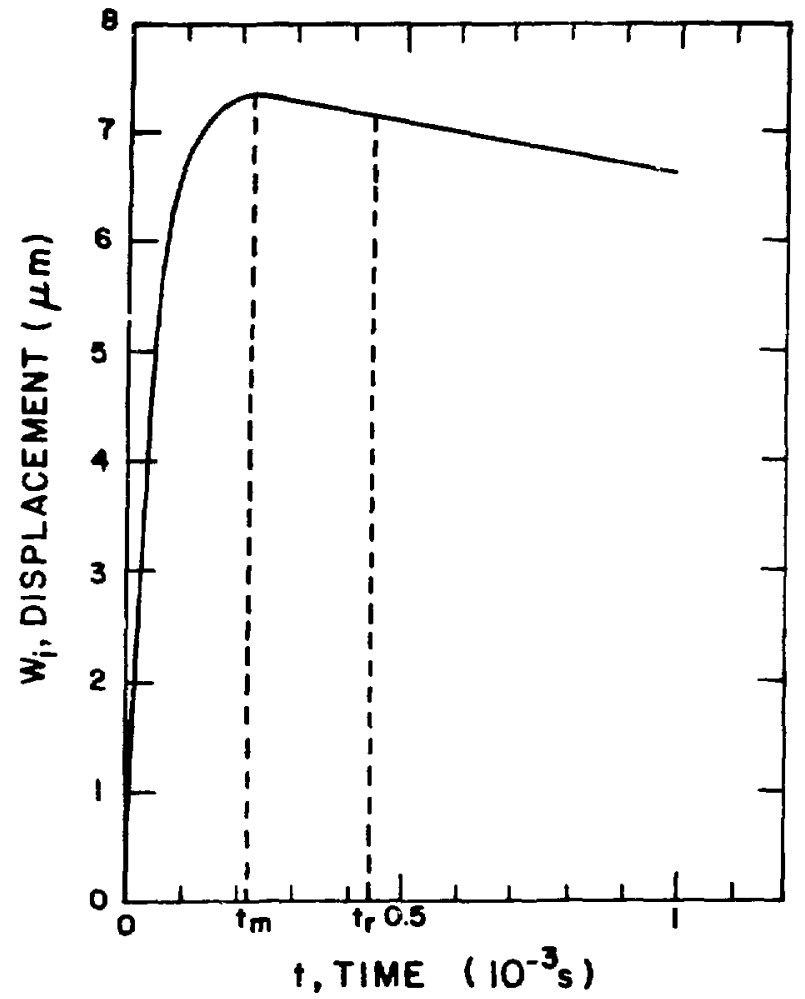

Fig. 45. Response of a structural shell in the presence of hydrodynamic coupling.

The shell relaxation time indicated by Fig. 45 is very long in comparison to the hydrodynamic wave transit time $\Delta / a_{\ell}$. Therefore, in some investigations, approximating the wave motion in the blanket with reflections from stationary shells may be justified.

Analysis of this problem requires simultaneous solution of Eq. (101) for the inner $(i=1)$ and for the outer $(i=2)$ shells and of the linearized wave equation describing the motion of the liquid between the shells.

$$
\frac{\partial^{2} u}{\partial t^{2}}-a_{\ell}^{2} \frac{\partial^{2} u}{\partial r^{2}}=0 \text {, }
$$

where $u$ is the local particle velocity in the liquid medium.

The initial conditions for $w_{1}$ at $t=0$ are given by $\mathrm{Eq} .(85)$ and for $w_{2}$ at $t=\Delta / a_{\ell}$ by

$$
w_{2}\left(\Delta a_{\ell}\right)=0 \text { and } \frac{d w_{2}\left(\Delta a_{\ell}\right)}{d \hat{t}}=\frac{1_{2}}{\Delta \delta_{2}} \text {, }
$$

where $I_{2}$ is the impulse transforted by the wave across the blanket to the outer shell. The initial and boundary conditions for EG. (110) are

$$
u \equiv 0 \text { at } t=0
$$

and

$$
u=d w_{j} / d t \text { at } r=R_{i}
$$

Equation (113) expresses the assumption qnat cavitation does not occur.

The pressure perturbation in the iquid blanket is given by the acoustic relations

$$
p= \pm o_{\ell} \dot{a}_{\ell} u
$$

where $(+)$ corresponds to outward and $(-)$ to inward traveling waves.

The system of equations specified above is solved easily by substituting for $u$ the genera? traveling-wave solution, which for the plane wave approximation is

$$
u=f\left(r-\partial_{Q} t\right)+G\left(r+a_{Q} t\right) \text {, }
$$

and by determining the functions $F$ and $G$ from initial and boundary conditions [Eas. (112) and (113)j combined with Eq. (102) for both shells. The results for different time intervals are presented separately.

$$
\text { 1. } 0<1<\Delta / a=t_{b}=\text { In this }
$$
time interval the outward (+) propagating wave has not yet reflected at $r=R_{2} ;$ therefore $G \equiv 0$ and

$$
\begin{aligned}
& u=c_{1}\left\{{ }_{-\omega_{1} e^{-\frac{\omega_{1}}{a_{l}}}\left[R_{l}-\left(r-a_{2} t\right)\right]}\right. \\
& \left.+w_{2} e^{-\frac{w_{2}}{a_{2}}\left[R_{j}-\left(r-a_{2} t\right)\right]}\right\} \text {. }
\end{aligned}
$$

$$
u \equiv 0, \quad r>a_{p} t
$$

The impulse delivered to the outer shell equals the integral of the pressure at $r=P_{2}$ from the time when the wave reflection begins, $t_{b}$, to the $t$ ime when it ends, $2 t_{b}$; that is, 


$$
I_{2}=\int_{t_{b}}^{2 t} p\left(R_{2}, t\right) d t .
$$

Substituting Eqs. (114) and (116) into (117), we easily calculate

$$
I_{2}=c_{\ell} a_{l} c_{1}\left(e^{-\omega_{1} t_{b}}-e^{-\omega_{2} t_{b}}\right) \text {. }
$$

A sample result illustrating the shape and amplitude of the propagating wave described by Eqs. (114) and (116) is shown in Fig. 46 for $1_{1}$ $=183$ dyne $\cdot \mathrm{s} / \mathrm{cm}^{2}$ and $\delta_{1}=\delta_{2}=1.5 \mathrm{~cm}$; the liquid is lithium $\left(o_{2}=0.53 \mathrm{~g} / \mathrm{cm}^{3}\right)$.

2. $\quad t_{b}<t<2 t_{b}-$ At time $t_{b}$, the head of the wave arrives at the outer shell ( $r$ $=R_{2}$ ) and begins to be reflected. In this process the front part of the wave accelerates the shell so that the remainder of the profile is reflected from a moving boundary. A rigorous determination of the motion precludes an explicit closed-form solution; however, the process $c$ an be modeled by postulating that a fraction $x$ of the impulse carried by the wave accelerates the shell and that the remainder $(1-x)$ is reflected. Accordingly, when $\mathrm{I}_{2}$ in $\mathrm{Eq} .(111)$ is replaced by $\mathrm{XI}_{2}$ the solution in the interval $t_{b} \leq t$ $\leq 2 t_{b}$ is

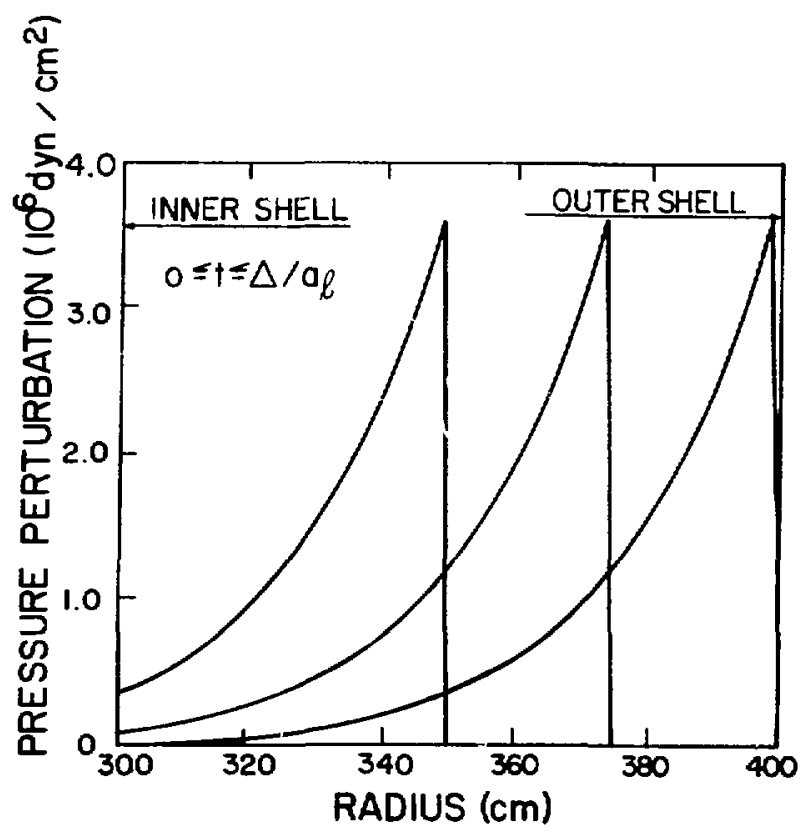

Fig. 46. Impulse-generated wave in the liquid blanket.

$$
\begin{aligned}
& u=c_{1}\left\{-\omega_{1} e^{-\frac{\omega_{1}}{a_{2}} R_{1}} e^{+\frac{\psi_{1}}{a_{2}}\left(r-a_{2} t\right)}\right. \\
& +\omega_{2} e^{-\frac{\omega_{2}}{a_{k}}} R_{1}+\frac{w_{2}}{a_{k}}\left(r-a_{2} t\right) \\
& +\omega_{1} e^{-\frac{\nu_{1}}{a_{l}}\left(R R^{\left.-2 R_{2}\right)}\right.} e^{-\frac{\nu_{1}}{a_{l}}\left(r+a_{2} t\right)} \\
& -w_{2} e^{-\frac{w_{2}}{a_{l}}\left(R_{1}-2 R_{2}\right)} e^{-\frac{w_{l}}{a_{l}}\left(r+a_{l} t\right)} \\
& +x C_{2}\left\{-\Omega_{1} e^{-\frac{\Omega_{1}}{a_{2}}\left(R_{1}-2 R_{2}\right)} e^{-\frac{\Omega_{1}}{a_{2}}\left(r+a_{l} t\right)}\right. \\
& +\Omega_{2} e^{-\frac{\Omega_{2}}{a_{l}}\left(R_{1}-2 R_{2}\right)} e^{-\frac{\Omega_{2}}{a_{2}}\left(r+a_{2} t\right)}
\end{aligned}
$$

Here $\Omega_{1}$ and $\Omega_{2}$ are the characteristic roots given by $\mathrm{Eq} .(103)$ for the outer sheji. $i=2$. In Eq. (119), the terms containing the variable $\left(r+a_{\ell} t\right)$ represent the reflected wave and therefore vanish identicaily for $r<R_{2}+\Delta-a_{2} t$.

of particular interest for the stress anajysis is the value of the impulse $1_{3}$ returned to the inner shell. It is given by the integral of the pressure at $r=R_{1}$ over the time interva] $2 t_{b} \leq t \leq 3 t_{b}$. Using Eq. (119) and the acoustic relations of $E q .(114)$, we obtain the simple expression

$$
\frac{I_{3}}{I_{2}}=1-\frac{\left.x_{2} i e^{-\Omega_{1} t_{b}}-e^{-\Omega_{2} t_{b}}\right)}{c_{1}\left(e^{-\omega_{1} t_{b}}-e^{-w_{2} t_{b}}\right)} .
$$

This expected result shows that the impuise $I_{3}$ returned to the inner shell approaches the value $I_{2}$ delivered by the wave to the outer shell as the amount of impulse absorbed by the outer shell diminishes $(x \rightarrow 0)$ because of increasing rigidity. Also, when the shelis are geometricaljy and elastically similar $\mathrm{CC}_{2} \approx \mathrm{C}_{7}$; 
$\left.\Omega_{1} \approx w_{1} ; \quad \Omega_{2} \approx w_{2}\right), \quad$ the impulse returned is diminished by the factor $1-x$, which, therefore, may be termed the reflection coefficient and $x$ the absorption coefficient.

The dependence of the reflected wave on the absorption coefficient $x$ is presented in Fig. 47 for the incident wave shown in Fig. 46. The result shows strong dependence, so $x$ must be known before the second impulse $c$ an be estimated. Unfortunately, we have not yet determined the appropriate value of $x$ analytically; it probably will depend on the relative magnitudes of the acoustic impedance, elastic stiffness, and inertia of the shell. We have, however, estimated the value of $x$ by comparing the analytic solution witli the as yet unpublished exact numerical calculations of the nonlinear blanket-shell interaction by R. R. Karpp of Los Alamos National Laboratory. The comparison shows that for the range of parameters of interest in ICF reactor studies, $x \approx 0.5$. The shape and the propagation of the reflected wave for that value of $x$ are illustrated in Fig. 48. With a known value of $x$, the successive wave reflections can be followed for as long as desired. However, this is best accomplished with a computer program because the algebra very rapidly becomes unmanageable.

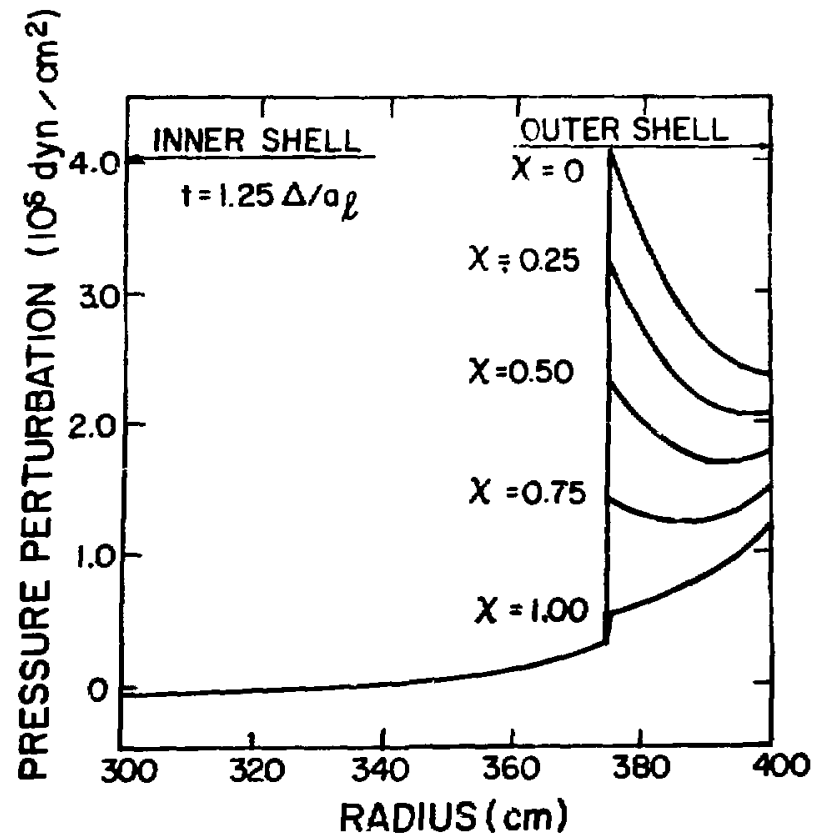

Fig. 47. Dependence of the reflected wave on the absorption coefficient $x$.

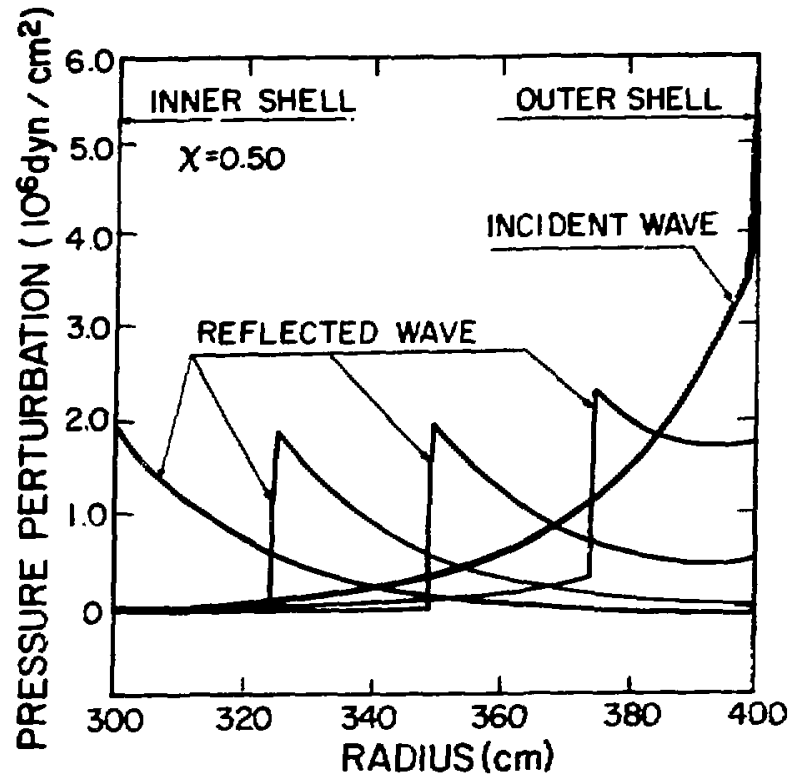

Fig. 48. Shape and propagation of reflected wave.

We close the discussion of impulse-generated wave motion with a few remarks about the effects of spherical geometry. To include the effect of spherical divergence in the analysis, Eq. (110) could be written and solved in spherical coordinates at a cost of more laborious algebraic operations, and the solution would be more complex. It is simpler to approximate the effect by superimposing on Eas. (116) and (119) the radial divergence and convergence as required. The results will be sufficiently accurate for mariy purposes when the blanket is not thick compared to the radius. It is also true that in one round trip of the wave the effects of the spherical divergence and convergence almost cance?.

\section{G. Stress Waves, Thermal Stress}

Thermal stresses arise in a structure because thermal distortions of its different parts are, in general, incompatible. In a thin shell with a steady-state linear temperature variation, the temperature difference $\Delta T$ across the faces induces the maximum stress at the surface given by 47,49

$$
\sigma=\frac{E a \Delta T}{2(T-v)}
$$


where $\alpha$ is the coefficient of linear thermal expansion. The factor 2 appears in the denominator because of the linear antisymetric stress distribution; that is, half of the shell thickness is in iension and half is in compression. In a thin surface layer with one side attached to inextendible $(E=0)$ bulk of material, the surface stress developed is 47,49

$$
\sigma=\frac{E \alpha \Delta T_{S}}{T-\nu},
$$

where $\Delta T_{S}$ is the instantaneous surface temperature increase.

The expression for the surface temperature increase $\Delta T_{s}$ to be used in Eq. (122) was developed in Sec. III (also Ref. 21) for the heatcapacity-dominated regime.

$$
\Delta T_{S}=\frac{Y x}{4 \pi R_{1}^{2} \delta o c_{1}}
$$

where $c_{1}$ is the heat capacity of the shell mate$r$ ial and $\delta$ is the depth of the heated surface layer. When the stress given by Eq. (122) is interpreted as the allowable value $\sigma_{m}$ and Eqs. (122) and (123) are combined, we obtain the radius-yield relation analogous to Eqs. (90), $(9 s),(95)$, and (98),

$$
R_{1}=\sqrt{\frac{E \alpha X Y}{8 \pi(T-v) \delta \alpha_{1} \sigma_{m}}} .
$$

Two comments about the above relation are in order. First, the estimate of $\Delta T_{s}$ given by Eq. (123) may be high because in its derivation no allowance was made for the fact that part of the energy $x y$ may be used up to melt and vaporize some of the material. Second, because the temperature increase persists for only approximately $10^{-9} \mathrm{~s}$ and is localized to a depth of less than a few micrometers, it is not clear that a catastrophic failure would occur if the surface material yielded locally or even melted for a short time. clearly, theoretical and experimental investigations are needed to determine allowable values of stress to be used in. Eq. (124) for the indicated loading characteristics.
A possible approach to the determination of allowable transient thermal stress is through the analysis of stress wave propagation in the vessel wall. A surface layer of depth $\delta$ heated sufficiently rapidly to a high temperature does not have time to expand and consequently experiences a compressive stress $\sigma_{0}$ that is relieved by a stress wave rather than by heat conduction. This can be seen from the following simple estimates. The characteristic time to propagate the effect elastically through a distance $\delta$ is given by $t_{w} \approx \delta / a_{i} \quad\left(a_{i}\right.$ is the wave speed in the wall materiall; for steel and $\delta \approx 10^{-4} \mathrm{~cm}$. $t_{w} \approx 2 \times 10^{-10} \mathrm{~s}$. The characteristic time for heat conduction is $t_{c} \approx \delta^{2} / x \quad(\kappa)$ is the coefficient of thermal diffusivity); for the same material and $\delta, \quad t_{c} \approx 2 \times 10^{-7} \mathrm{~s}$. Hence the effect of the thermal pulse will propagate elastically approximately a thousand times faster than by conduction.

Instead of giving a standard mathematical description of wave propagation, we sumarize graphically in $F$ ig. 49 the characteristics of thermally excited stress waves. Shown are the initial compressive stress $\sigma_{0}$ induced by the temperature increase $\Delta T_{S}$ in the surface layer of depth $\delta$, the restiting stress during reflection from the inner face of the wall, and the same wave at the time $\delta / a_{j}$ when the reflection process is completed. The resulting wave propacating through the wall consists of a compression chase

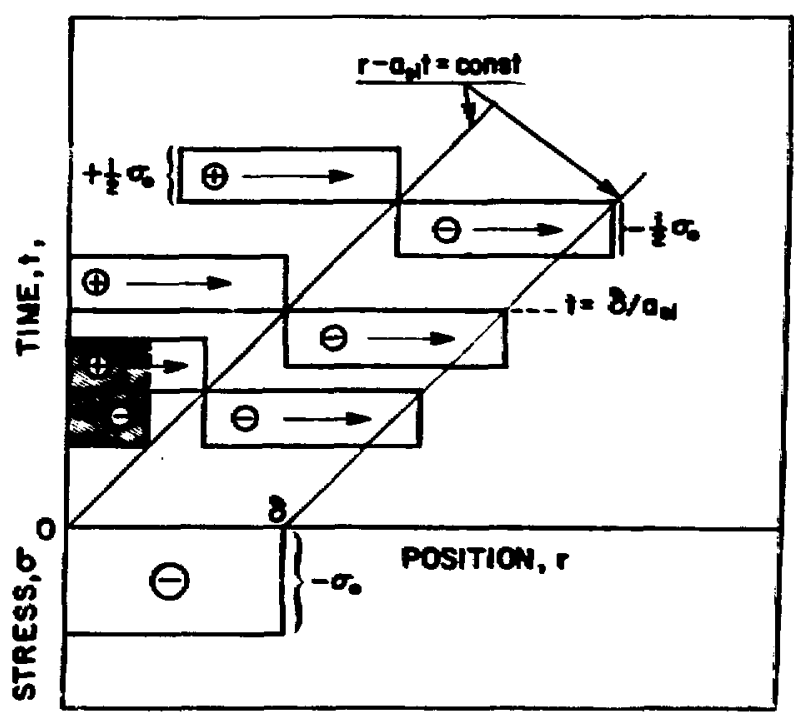

Fig. 49. Stress wave propagation: 4 tensile, - compressive. 
of length $\delta$, followed by an equally long tensile phase, the amplitudes of both phases being equal to $a_{0} / 2$. Clearly, this wave produces tensile and compressive stresses equal to $\sigma_{0} / 2$ at the inner and outer faces of the wall and, therefore, its amplitude should be limited to an allowable stress level to avoid spallation.

To resolve uncertainties indicated in the comments following Eq. (124), J. O. Jacobson (LoS Alamos National Laworatory) has been investigating numerically the characteristics and propagation of thermally generated stress waves by using model constitutive relations that admit phase transitions and tensile strength in liquid phases (for example, rillotson, Redlich-kwang). Even though the study is far from complete, preliminary results suggest certain conclusions. First, the magnitude of the impulse generated with the blowoff of wall material appears to be independent of the nonequilibrium effects at phase changes of the equation of state and of the spall strength of the material. Therefore, Eq. (89) (with ine proper value of $n$ ) is a valid estiriate of the impulse. Second, the rapid temperature and pressure in. creases in the thin layer into which the energy is deposited send a shork wave into ihe cold material, with the pressure behind it equal to approximately one-half the value in the hot layer. This value exceeds the yield strength of the shocked material. Third, the shock is attenuated very rapidly; its amplitude decreases by more than half in $16 \mathrm{um}(3 \mathrm{~ns})$. This is a consequence of the cumulative effect of ma.y short wavelengths (21 $\mathrm{lm})$. which is nonnegligible when the ratio $a_{i}{ }_{i} / 2 x$ is large 50 (this is the . case in ICF reactor studies) even though the thermoelastic coupling constant and, therefore, the logarithmic decrement are smal?.

\section{H. Fatique}

Cyclic operation of ICF reactors implies that fatigue strength (with appropriate satety factors) should be used as the allowable working stress. Fatigue strength is determined by threshold values of stress concentrations at microscopic fatigue cracks and equals that value of the stress at which the growtn -ate of the size of fatigue cracks vanishes for all practical purposes.
If thermally generated elastic stress waves are determined to be the governing phenomenon in the design of ICF reactor vesseis [Eq. [124)], then the usual fatique strengths of materials may be increased because of extreme shortness of stress wavelengths of these waves. In a recerit paper, weertman 51 pointed out that current theories of fatioue failure may not be valid whesi the stress wavelength is sinaller inan the fat aque crack and proposed an appropriate formulation. Results of his modified formulation indicate that increased levels of lluctuating stresses may be tolerated when stress wavelengths are very small. This area requires additional stucy.

\section{Concluding Remarks}

In the analysis of loads and stresses presented in this seciion, we separated the difierent effects and investigated each in isciation. Such a procedure helps to abtain insignts into enc estimates of the dominant effects and is justifieo when the snell response is in the linear lejastu: regine where the superposition principle riay de applicaole. in reality, however, all effects are present simultaneously, ani the transition of dowinunce from one effect to another occurs not abruptly but gradually; therefore, it is desirabi. to investigate certain combinations of effects 10 understand better the respunse behavior in iransi$t$ ion regimes.

An example of such transition is shown in Fiy. 50, which is the plot ut strain cevelopec in the walls of a spherica) reactor vesse] (shown in Fig. 34) for increasing buffer gas censities inside the inner shell. This result has been obtained by R. R. Karpp (Los Alamos vatjona) Laboratory) by numerically integrating ine coupled equations of gas dynamics (inside the jnner shell), elastic stiell response, and hydrodynamics (between the shells). In the calculation the shell radii were 200 and $300 \mathrm{~cm}$, the shel] thicknesses were $1 \mathrm{~cm}$, and the material was steel. The pressure pulse was generated with a $30-r i j$ energy release from an explosive charge of $0.5 \mathrm{~g}$; the aoiabatic exponent of the gas was 1.2 . The curves in Fig. 50 show that at low buffer gas densities o the debris impact dominates and therefore the strain is approximateiy independent of the 


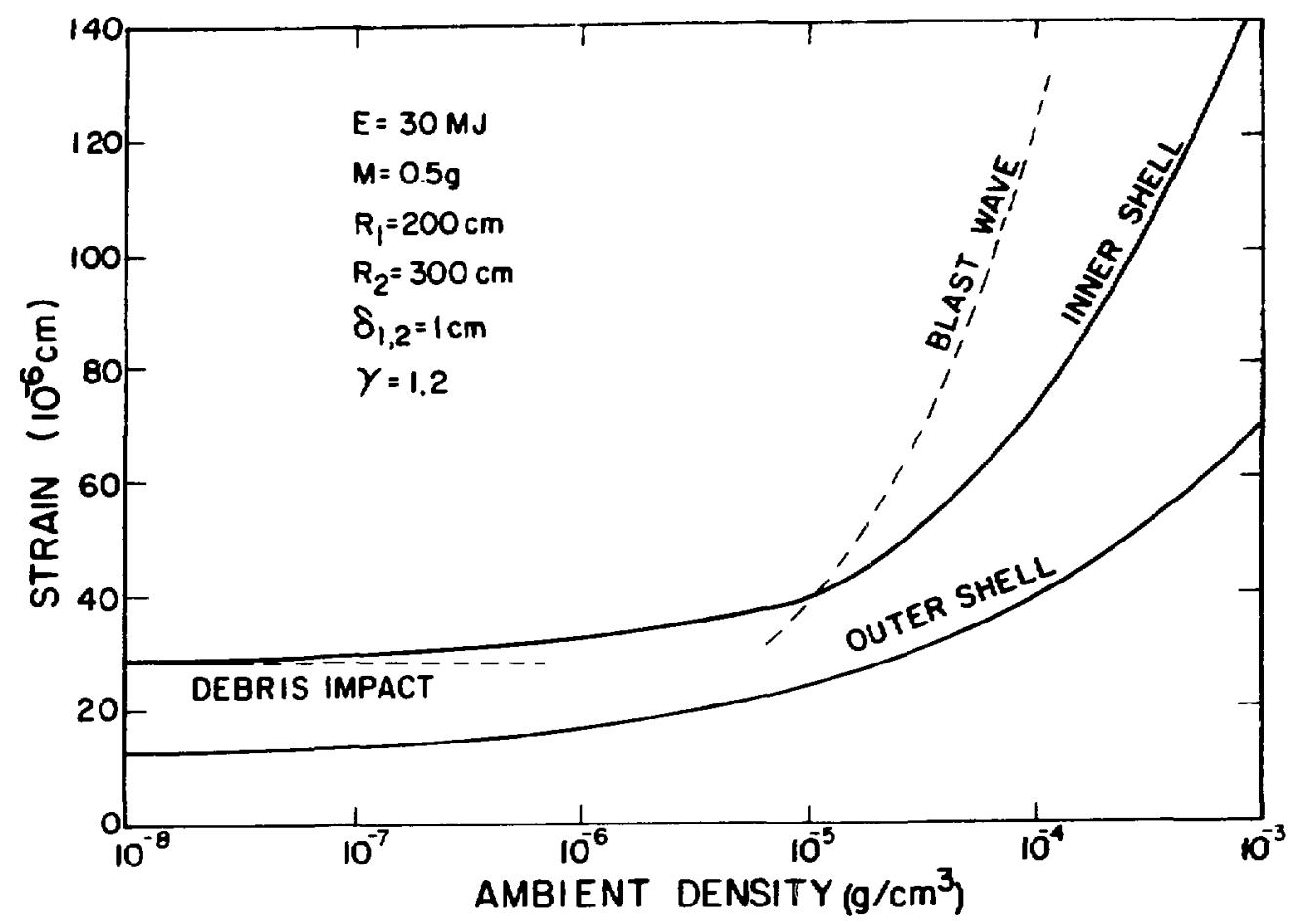

Fig. 50. Dependence of strain on ambient background gas density.

density [Eqs. (87) and (92)]; at higher densities the strain increases with $o_{0}$ but not as rapidly as predicted by the blast wave theory $\left[\sqrt{D_{0}}\right.$, cf. Eqs. (87) and (94)]. Thus, the calculation shows that for the conditions anticipated inside ICF reactor cavities the blast wave theory will overestimate the wall impulse. Consequently, the modified solution of Freiwald and Axford ${ }^{19}$ should be used to obtain accurate load estimates. Part of the reduction of strain below values predicted by the blast wave theory is attributable to the presence of liquid lithium between the shells as determined in Sec. V.F.

\section{SYSTEMS INTEGRATION}

\section{A. Introduction}

Successful commercialization of ICF requires integration of the subsystems listed in Sec. I into the functional, reliable, and efficient electric power generating plant shown in Fig. 51 . The procedure should take into account the fact that, at present, the scientific feasibility of this process has not yet been established and the ICF progran is in its research phase. Therefore,
ICF reactor systems integration efforts must interact with research and technology development activities. In this process research results are used to arrive at feasible systems configurations and to deduce functional relationships among parameters characterizing different components (scaling laws). The scaling laws are used to infer the tradeoffs, which, in turn, indicate directions of greatest payoff for the research and development activities. In this way, the systens and technology requirements can be made increasingly more precise.

Systems integration is analogous to solving a variational problem with boundary constraints. one boundary constraint is the research result that specifies the relationship between pulse energy of the driver beam and yield of the fuel pellet. Because the ratio of the pellet yield to the beam-pulse energy is called the pellet gain, this relationship is called the gain curve. The second boundary constraint is the desirable or at least economically acceptable size (that is, capacity) of the power plant, which will be determined by market analyses and experience of power utili$t$ ies. An integrated functional power plant should 


\section{ELECTRIC POWER GENERATION REQUIRES INTEGRATION OF UNIQUE ICF SUBSYSTEMS WITH CONYENTIONAL POWER PLANT TECHNOLOGY}

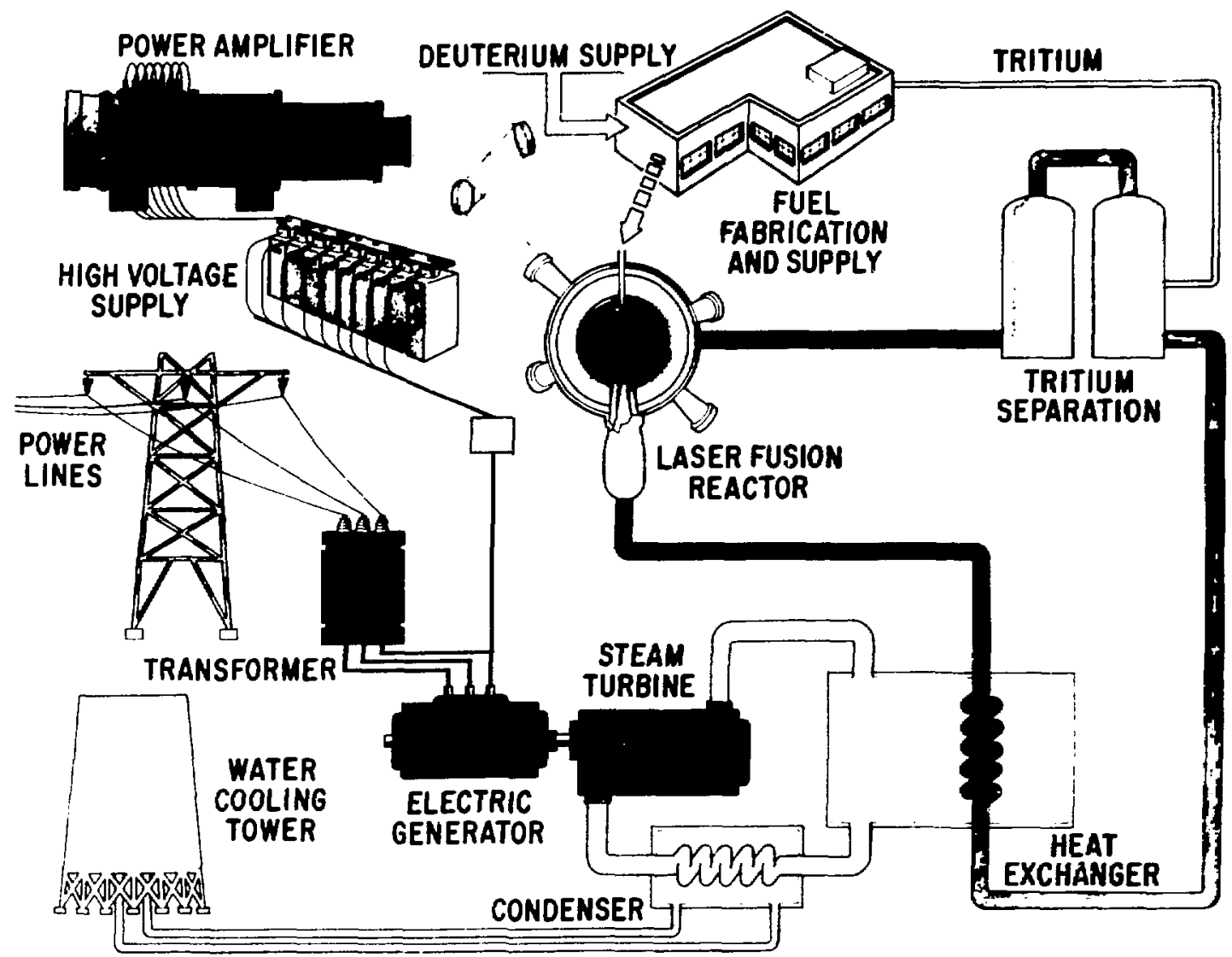

Fig. 51. ICF electric power plant elements.

satisfy these boundary constraints and, in addition, should minimize an objective function (for example, the levelized busbar cost of power or some other economic indicator).

The process of systems integration and optimization also must be subjected to the additional constraints of the subsystems' performance characteristics dictated by the availability of the necessary technologies. Investigations of the dependence of the value of the objective function on these constraints determine the potential payoffs from technology developments.

Following the analogy with the variational problem, finding a solution should begin with the establishment of its existence. As in most practical problems, it is advisable to establish this existence with a construction; in the present case, with a point design. Successive variatinns then can be performed exploiting the scaling laws to reduce the value of the objective function. Although this process may not necessarily converge to a unique configuration, it will lead to an economically competitive or acceptable design and will indicate possible tracieoffs.

\section{B. Systems Integrat ion Procedure}

The bases for the systems integration into a point design of an electric power generating station and for successive optimizations are energy and mass flows within the power plant. The principal energy paths, indicated in Fig. 52, are 


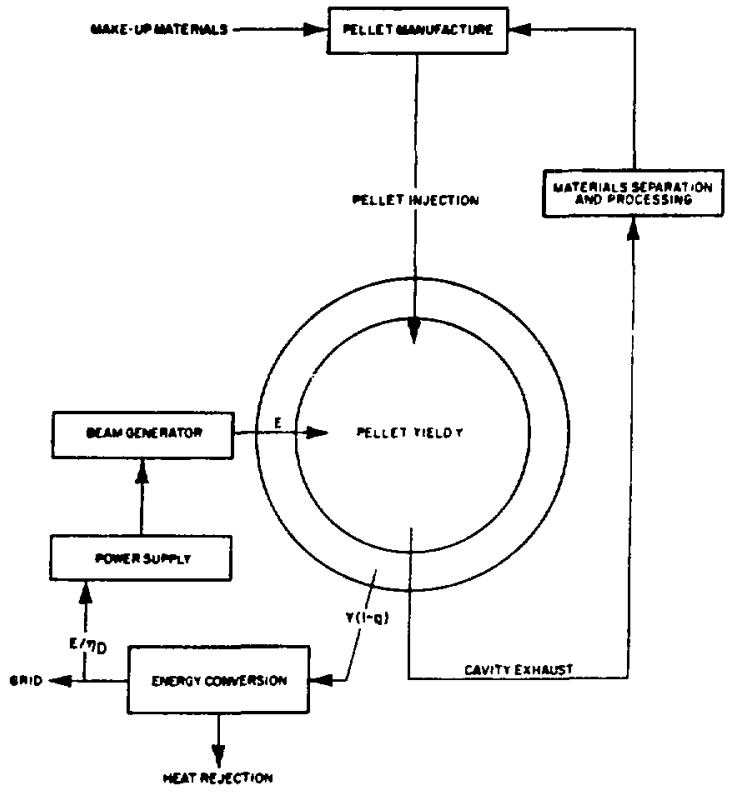

Fig. 52. Energy and mass flows in an ICF reactor.

- net energy production path (fuel-pellet yield - energy conversion - energy diversion, for example, beam power supply $\rightarrow$ heat rejection - power grid);

- driver circuit (beam-pulse energy + fuelpellet yield $\rightarrow$ energy converter $\rightarrow$ beam power supply $\rightarrow$ beam-pulse energy); and

- ancillary equipment branch (fuel-pellet yield - energy converter $\rightarrow$ ancillary equipment; for example, pumps, controls, separators).

The principal mass flow circuits (Fig. 52) are

- the primary heat transfer loop (reactor vessel $\rightarrow$ steam generator or heat exchanger $\rightarrow$ reactor vessel);

- the secondary heat transfer loop (steam generator $\rightarrow$ turbine + condensor $\rightarrow$ steam generator ); and

- the fuel supply path (pellet injection $\rightarrow$ products separation $\rightarrow$ proces sing $\rightarrow$ fuel - pellet manufacture $\rightarrow$ fuelpellet injection).

The mass flow rates in the primary and secondary loops are determined not only by the required heat transfer rates, but also by the characteristics of the fluid carriers.

The system integration procedure consists of writing the energy and mass transfer rates that are mutually consistent and satisfy the boundary conditions for each path; these are the relations that the system parameters must satisfy. They must reflect not only' the characteristics of each path element but aiso of the interfaces between individual elements (for example, reactor cavitydriver interface). Examinations of these mutual dependencies and the sensitivity of the value of the objective function to changes in characteristic system parameters indicate directions of parameter variations that lead toward design optimization.

System integration is carried out most conveniently with computer modeling, which allows inclusion of rany more details than would be otherwise possible. However, it is also possible and useful to develop insights into ICF reactor systems integration and to derive trends by using simple analytic considerations. This approach is illustrated in the present section. The results ottained may be used as general technical guidelines for parametric system optimizations.

1. The Model. The subsequent analysis is based on the postulate that variations in reactor cost with pellet $y$ ield and driver pulse energy are dominated by changes in the vessel and driver costs.

We have shown previously 52 that the capita? cost of the reactor vessel is proportional to the $3 / 2$ power of the pellet yield $Y$. More detailed cost studies 53 show that the exponent in this cost proportionality is less than $3 / 2$ but greater than unity, which is sufficiunt for the validity of the conclusions derived below.

cost studies indicate that a proportionality to the $\alpha$ power of the pulse energy $E$ may approximate the driver cost $w$ ith adequate accuracy; the economy of scale exponent $a$ is most likely to be in the range $0.4 \leq a \leq 1.0$.

The gain curve usually is given as

$$
Y=Y_{0} E^{\beta},
$$


with $Y_{0}$ and $B$ constant and $\beta>1$. In the following analysis, this relation is used also in the form

$$
E=K Y^{1 / B} \text {, }
$$

where $K$ is another constant related to $Y_{0}$ and B.

In reference to Fig. 52, energy exceeding that required to operate the plant produced by each pellet microexplosion is given by $\mathrm{Y}_{\mathrm{T}}(1$ - q) $-E / n_{D}$, where $n_{T}$ is the conversion efficiency, $q$ is the fraction of energy lost through the structure, and $n_{D}$ is the driver efficiency.

Using the above expressions for the costs of reactor vessel and driver and eliminating $E$ in favor of $Y$ by Eq. (126), we obtain the following expression for the unit capital cost of power $u$ that is the objective function in further analysis.

$$
u=\frac{n C_{V} \sqrt{Y}+C_{D} Y^{\alpha / \beta-1}}{N\left[\eta_{T}(1-q)-\left(K / \eta_{D}\right) Y^{1 / \beta-1}\right]} \text {, }
$$

where $n$ is the number of reactor vessels, $N$ is the driver repetition frequency, and $C_{V}$ and $C_{D}$ are cost constants.

2. Effect of Gain Curve, Boundary Constraints. We analyze the effect of the gain curve on the average unit cost of power and use the results to demonstrate that the boundary constraints play an essential role in the determination of parametric dependencies (trends) and therefore must be included and discussed in all optimization studies.

When studying the effects of the gain curve, it is customary to make comparisons for a constant pulse energy, E. ${ }^{54}$ In our model, making these comparisons corresponds to determining the variation of the pellet yield $Y$ with $B$ from Eq. (125) for constant $Y_{0}$ and $E$. (We assume that varia$t$ ion of the gain curve is modeled by variation in the gain exponent $B$. )

The denominator in Eq. (127) changes slowly with $Y$ both because the recirculating fraction $\left(E / T_{D}\right)$ is small and because it is weakiy dependent on $Y$ (since the exponent is small, that is, in general, $\beta$ is not far from unity).
Therefore, the variation of $u$ with $B$ is given primarily by variations of the numerator. The calculation of that variation using Eq. (125) results in the following expression for the change in unit cost $\delta u$ corresponding to the change $\delta \beta$.

$$
\begin{gathered}
\delta u=\left\{\frac{1}{2}\left(\mathrm{nC}_{V}, \bar{Y}\right) \ln \right. \\
\left.-\left(C_{D} y^{\alpha / \beta-1}\right)\left[\left(1-\frac{\alpha}{\beta}\right) \ln E+\frac{\alpha}{\beta^{2}} \ln Y\right]\right\} \delta B .
\end{gathered}
$$

In general, the driver cost $\left(C_{D}\right)$ dominates the reactor cost and $\alpha / \beta<1$; therefore, Eq. (128) shows that the unit cost decreases as $B$ increases and the amount of decrease is proportional to the cost. This result was expected. Because of the economy of scale, the driver cost per unit power output decreases as $Y$ increases with $B$ for constant pulse energy, $E$. However, because of the diseconomy of scale in reactor vessel cost (exponent greater than unity), the unit cost would increase with increasing $B$ (that is, $Y$ ) if the vessel cost was jominant. This result also is evident from $E q .(128)$. This behavior is confirmed by the results of numerical modeling with the TROFAN code 52 and is illustrated in Fig. 53.

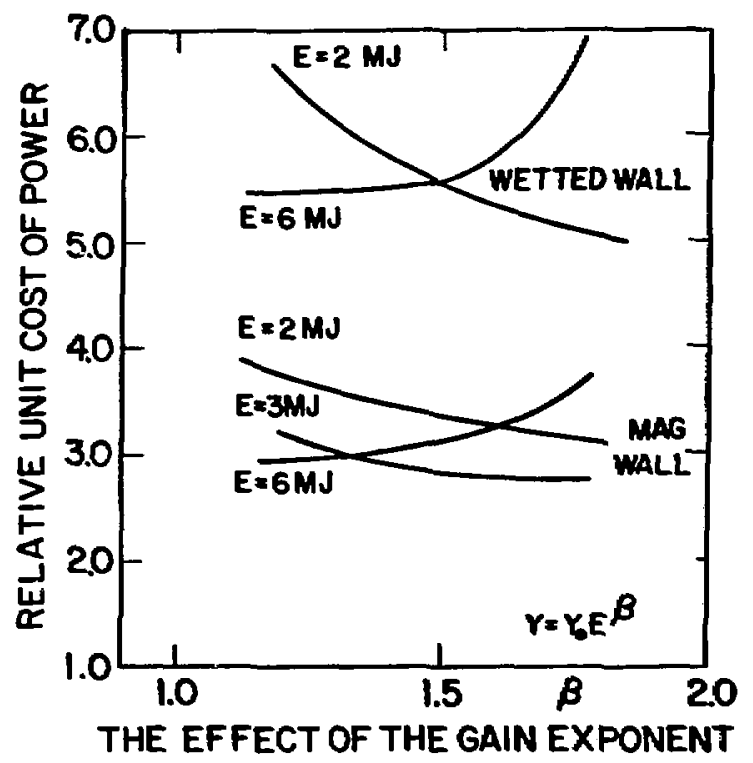

Fig. 53. The effect of the gain exponent on unit cost of power 
To illustrate the essential nature of boundary constraints in parameter optimizations, we now recalculate the effect of changing $B$ on the unit power cost when the net power output is held constant. For a constant driver repetition frequency $N$, the requirement of constant power [see Fig. 52 or the expression following Eq. (126)] implies a functional relationship $Y(B)$ from which we easily calculate the expression

$$
\left[Y \eta_{T}(1-q)-\frac{E}{B \eta_{D}}\right] \frac{d Y}{d B}=-\frac{K}{B^{2} \eta_{D}} Y^{l / \beta+1} \ln Y .
$$

This result indicates that $d y / d \beta$ is always negative, which means that, to maintain a constant net power output, the yield $Y$ must be decreased as the steepness of the gain curve increases because of the decrease in the recirculating power fraction. This is an intuitively anticipated result.

The effect of a change in $\beta$ on $u$ now can be inferred from Eq. (127) directly without additional calculations. A decrease in the yield $Y$ with an increase in $\beta(\mathrm{dY} / \mathrm{d} \beta<0)$ implies an increase in the unit cost (because $\alpha / \beta<1$ ) when the driver cost dominates (as it usually does) because of the economy of scale in driver cost. (The opposite would be true if the reactor cost was dominated by the vessel cost for which there is a diseconomy of scale). This as marently counterintuitive conclusion may be a consequence of the omission of the balance of the plant from the analysis. For constant ret power output, a decrease in the recirculating power fraction implies a smaller and less expensive turbogenerator. Whether the resulting reduction in cost is sifficient to overcome the effect of the increased unit driver cost will depend on relative magnitudes and scalings of these two costs.

These simple considerations show that the change in the unit capital cost with changing slope of the gain curve $B$ can be either downward or upward, depending on whether the driver pulse energy $E$ or the reactor power output is kept constant, and thus demonstrate the essential dependence of optimization on boundary constraints.
3. Optimal Pellet vield. It is useful to begin the ICF reactor system studies with a der $\hat{j}-$ vation of guidelines pertaining to the desirable size of the pellet yield and of the driver to be developed. This is accomplished easily by using Eq. (127). When the denominator does not vary significantly, the minimum of the numerator for a constant number of vessels $n$ occurs a $i$

$$
Y_{m}=\left[2\left(1-\frac{a}{\beta}\right) \frac{C_{D}}{n C_{V}}\right]^{\frac{2}{3-2 \alpha / \beta}} .
$$

This result shows that the value of the pellet yield $Y_{m}$, which minimizes the average unit power cost, depends only on the ratio of the economy of scale to gain exponents, $\alpha / B$, and on the ratio of the cost constants, $c_{0} / n C_{v}$. In general, the values of the ratio $\alpha / B$ are near $1 / 2$, so that the exponent in Eq. (130) is near unity. Therefore, $Y_{m}$ increases approximately 3 inearly with the ratio of the cost constants.

The variation of the optimal pellet yield in the relevant range of parameters is shown in Fig. 54. For relatively high driver costs and $h$ igh values of $\alpha / \beta$ (that is, either large $\alpha$, meaning little economy of scale, or small $B$, meaning shallow gain curve), the values of ${ }_{\mathrm{in}}$ become unmanageably high. However, for values of

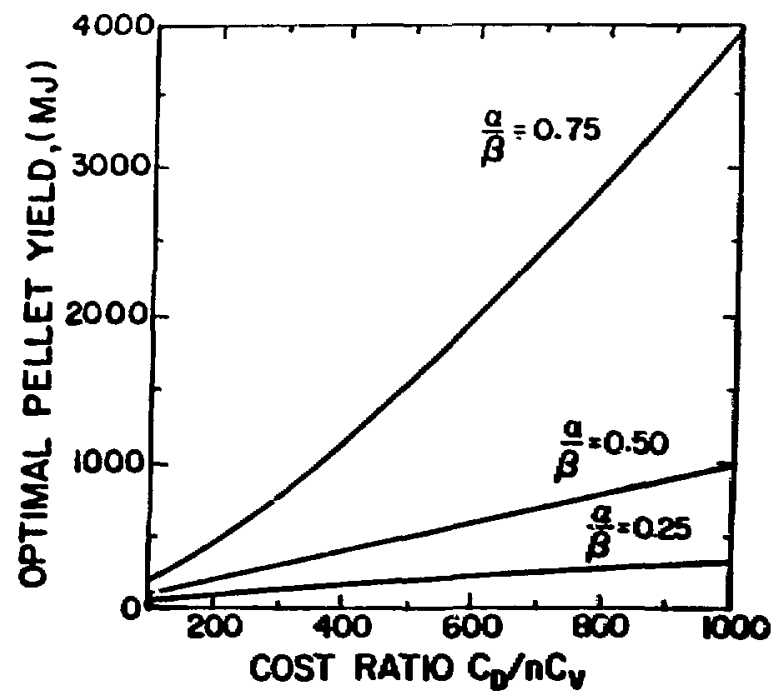

Fig. 54. Variation of optimal pellet yield. 
$\alpha / B$ near $1 / 2$ and lower, the optimal yield is acceptable for any value of the cost ratio.

For representative values $\alpha=0.6, \quad \beta$ $=1.2, \quad C_{D}=350 \mathrm{M \$}$, and $\quad n C_{V}=2 \mathrm{MS}$, the optimal yield is $175 \mathrm{MJ}$. This value of $y_{m}$ agrees closely with the results obtained with detailed computer modeling using the TROFAN Code $^{52}$ for the reactor design that uses wetted wall containment vessels. 55

4. Reactor Configuration. For a given power plant output, one of the first questions to be answered is, Should the reactor consist of one large or several small containment vessels? The answer is readily determined from the previously introduced expressions for the costs of the reactor vessel and the driver.

The vessel cost for a one-vessel design is

$$
c(1)=C_{V} y^{3 / 2}
$$

If there is no technical constraint precluding the possibility of increasing the driver repetition frequency to the required level, the vessel cost for an n-cavity design is

$$
c(n)=n C_{V}(y / n)^{3 / 2}=c(1) / \sqrt{n},
$$

because the yield per cavity is $Y / n$. Therefore, it is advantageous to use several small cavities in place of one large cavity, provided the resulting system complexity (for example, piping and beam switching) does not negate the above scaling.

The corresponding saving in driver cost is determined analogousiy. The driver cost for a one-cavity design is

$$
D(1)=C_{0} Y^{\alpha / \beta},
$$

and for an n-cavity design it is

$$
D(n)=C_{D}(Y / n)^{\alpha / \beta}=n^{-\alpha / \beta} D(1) .
$$

(Note that in this case the economy-of-scale argument does not apply because the driver repetition rate does not remain constant.) Because $\alpha / \beta$ $>0$, the above result indicates a cost reduction that will be realizable provided it is not cancelled by the required increase in the frequency capability and system complexity resulting from the addition of beam-switching devices.

In the derivation of the above simple cost estimates, the net power output remained constant. Consequently, the design policy of using several small containment vessels in place of one large vessel will lower the average capital cost of power, which is one objective of engineering opt imizations.

5. Containment Vessel size, Lifet ime Tradeoff. The lifetime of an ICF reactor vessel is governed, in general, either by the neutron fluence or by the material loss, both of which are inversely proportional to the first-wall surface area. Therefore, it is natural to look for a tradeoff between vessel size and its lifetime. The required analysis is illustrated with the derivation of the answer to a specific question. If the expenses associated with the need for $n$ containment vessel replacements during the plant lifetime can be eliminated by increasing its size, how large can the increase be before it is no longer cost effective?

A plausible estimate of the reactor vessel cost with necessary replacements is

$$
C(n)=C_{V} R^{2} d(1+n r) t_{o} / t_{p},
$$

where $C_{V}$ is a constant, $R$ is the vessel radius, $d$ is its wall thickness, $r$ is the fractional replacement cost (including installation), and $t_{p} / t_{o}$ is the productive fraction of the reactor lifetime $t_{D}$. The cost of a large vessel not requiring replacements is

$$
C(0)=C_{y} R_{0}^{2} d 0
$$

it will be cost effective, provided the inequality

$$
\frac{R_{0}^{2} d_{0}}{R^{2} d} \leq \frac{t_{0}}{t_{D}}(1+n r)
$$

is satisfied.

This analysis can be refined in several ways. One way is to postulate that the number of replacements $n$ is inversely proportional to the 
surface area $4 \pi R^{2}$ and to derive a corresponding estimate for the productive fraction $t_{p} / t_{0}$. However, the inequality derived above suffices to indicate the approach.

We illustrate the result with an example. Suppose the containment vessel has to be replaced every 3 years during a reactor lifetime of 30 years (that is, $n=10$ ). Suppose further that only the first and the inner structural walls need be replaced at $30 \%$ of the original reactor vessel cost (that is, $r=0.30$ ) and that $10 \%$ of the lifetime must be devoted to replacement activities (that is, $\left.t_{p} / t_{0}=0.90\right)$. If, in addition, we postulate for the sake of definiteness that $d_{0}$ $=d$, then the inequality reduces to

$$
R_{0} \leq 2.7 R
$$

The result means that increasing the radius by any factor less than 2 to avoid replacements is cost effective; if, however, the radius must be more than doubled to avoid replacements, then it is not cost effective.

\section{Concluding Remarks}

The discussions of trends and tradeoffs presented in this section are not intended to be complete and exhaustive, but only to illustrate one possible approach for arriving at a starting point of a design and for indicating directions of parameter variations that may reduce the value of the objective function. Analyses of this kind, when used judiciously, will provide insights into parametric interdependencies and will contribute to the understanding, appreciation, and proper assignment of priorities to technical problems encountered in the development of ICF reactors.

The technical, economic, and operating characteristics of complete central electric power generating stations can be modeled acceptably in detail only with computer codes such as the previously mentioned TROFAN (Tradeoff and Analysis). 56,57 The explicit results derived and illustrated in this section are intended to supplement the computer modelings and to save time and computational effort by indicating trends and sensitivities to be exploited in optimization studies.

\section{ACKNOWLEDGMENTS}

This report contains descriptions of models of ICF reactor components, methods of analysis, and selected analytical results that have been developed at the Los Alamos National Laboratory during the past several years. Most of the analytical development presented is the work of the author; however, ICF systems studies at Los Alamos have been conducted by a multidisciplinary team and have benefited from many contributors. The conceptual reactor designs and many of the approaches to their analysis have been developed through discussions and evaluations by team members. The stimulating environment thus created has been essential to the development of the material included in this report.

Contributors who deserve particular recognition include: L. A. Booth and T. G. Frank, the Group Leader and Assistant Group Leader when this work was carried out, and $\mathrm{J}$. J. Devaney, D. 0. Dickman, J. C. Goldstein, J. F. Hafer, A. R. Larson, J. H. Pendergrass, and W. A. Reupke. Some of the calculations were carried out by $P$. L. Rood and the final typing and production were handled by J. I. Stelzer.

\section{REFERENCES}

1. I. 0. Bohachevsky, J. F. Hafer, J. J. Devaney, and J. H. Pendergrass, "Inertial Confinement Fusion Reactor Cavity Phenomena," Proc. Am. Nucl. Soc. Topical Meeting on Tech. of Controlled Nuclear Fusion, 3rd, Santa Fe, NM, May 9-11, 1978 (National Technica? Information Service, US Dept. of Commerce, 1978) CONF-780508, Yol. 2, p. 1167.

2. J. L. Delcroix, Introduction to the Theory of Ionized Gases (Interscience Publishers, New York, 1960), pp. 19-22.

3. S. Chapman and T. G. Cowling, The Mathematical Theory of Nonun iform Gases TCambridge University Press, London, 1952), pp. 220, 252.

4. R. W. Conn, S. I. Abde T-Khalik, G. A. Moses, F. Beranek, E. T. Cheng, G. W. Cooper, R. B. Dro 11, T. Henderson, J. Howard, T. O. Hunter, E. M. Larsen, G. L. Kulcinski, E. G. Lovell, G. R. Mageissen, C. H. Maynard, K. R. Oku Ia, M. Ortman, M. M. H. Ragheb, W. B. Rensel, D. Solomon, R. L. Spencer, I. H. Sviatos lavsky, H. F. Vogelsang, R. D. Watson, and H. G. Wolfer, "SOLASE, a Conceptual Laser Fusion Reactor Design," University of Hisconsin report UWFDA-220 (December 1977). 
5. D. 0. Dickman, R. L. Morse, and C. W. Nielson, "Numerical Simulation of Axisymmetric, Collisionless, Finite- $\beta$ Plasma," Phys. Fluids 12, 1708-1716 (1969).

6. J. C. Goldstein, I. O. Bohachevsky, and D. 0 . Dickman, "Ion Motion in Laser Fusion Reactor Studies," Bull. Am. Phys. Soc. Paper 9p7, Series 11, 21, 1185 (1976).

7. R. R. Peterson and G. A. Moses, "MFP--A Calculation of Radiation Mean Free Paths, Ionization and Internal Energies in Noble Gases," Fusion Engineering Program, University of Wisconsin report UWFDM-307 (September 1979).

8. J. W. Shearer and W. S. Barnes, "Numerical Calculations of Plasma Heating by Means of Subnanosecond Laser Pulses," in Laser Interaction and Related Plasma Phenomena, $\mathrm{H}$. J. Schwarz and H. Hora, Eds. (Plenum Press, New York, 1971).

9. P. D. Lax, "Weak Solutions of Nonlinear Hyperbolic Equations and Their Numerical Computation," Comm. Pure and Appl. Math. VII, 159193 (1954).

10. I. 0. Bohachevsky and E. L. Rubin, "A Direct Method for Computation of Nonequilibrium Flows with Detached Shock Waves," AIAA Journ. 4, 600-607 (1966).

11. I. 0. Bohachersky and R. E. Mates, "A Direct Method for Calculation of the Flow About an Axisymmetric Blunt Body at an Angle of Attack," AIAA Journ. 4, 776-782 (1966).

12. I. 0. Bohachevsky and R. N. Kostoff, "Supersonic Flow Over Convex and Concave Shapes with Radiation and Ablation Effects," AIAA Journ. 8, 1024-1031 (1972).

13. J. J. Devaney, "First-Wall Magnetic Protection in an Inertially Confined Thermonuclear Reactor," Los Alamos National Laboratory report LA-8286-MS (May 1980).

14. H. A. Bethe, K. Fuchs, J. 0. Hirschfelder, J. L. Magee, R. E. Peier $1 \mathrm{~s}$, and J. von Neumann, "Blast Wave," Los Alamos National Laboratory report LA-2000 (March 1958).

15. Ya. B. Zel'dovich and Yu. P. Raizer, Physics of Shock Waves and High-Temperature Hydrodynamic Phenomena, $W$. 0 . Hayes and $R$. $F$. Probstein, Eds. (Academic Press, New York, 1967), Chapt. IX, pp. 598-684.

16. H. A. Bethe, "Theory of the Fireball," Los Alamos National Laboratory report LA-3064 (June 1964).

17. R. R. Peterson and G. A. Moses, "Blast Wave Calculations in Argon Cavity Gas for Light Ion Beam Fusion Reactors," University of Wiscons in report UWFOM-315 (October 1979).

18. V. P. Korobejnikov, Problems in the Theory of Point Explosion in Gases (Nauka, Moscow, 1973).
19. D. A. Freiwald and R. A. Axford, "Approximate Spherical Blast Theory Including Source Mass," J. App 1. Phys. 46, No. 3, 1171-1174 (1975).

20. Warren M. Rohsenow, Part A, Film Condensation in Sec. 12, Condensation; Handbook of Heat Transfer (McGraw-Hill, New York, 1973).

21. T. G. Frank, I. 0. Bohachersky, L. A. Booth, and J. H. Pendergrass, "Heat Transfer Problems Associated with Laser Fusion," AIChE Symp. Series, Vol. 73, No. 168, pp. 77-85 (1977).

22. H. S. Carslaw and J. C. Jaeger, Conduction of Heat in Solids, 2nd ed. (Oxford University Press, England, 1950), p. 57.

23. J. L. Craston, R. Hancox, A. E. Robson, S. Kaufman, H. T. Miles, A. A. ware, and J. A. Wesson, "The Role of Materials in Controlled Thermonuclear Research," Proc. Int. Conf. Peaceful Uses At. Energy, 2nd, Geneva, September 1-13, 1958 (United Nations, 1958), vol. 32, pp. 414.

24. R. Behrisch, "First-Wall Erosion in Fusion Reactors," Nucl. Fusion 12, 695 (1972).

25. R. A. Axford, "Direct Evaluation of Transient Surface Temperatures and Heat Fluxes," Los Alamos National Laboratory report LA-6051 (December 197j).

26. D. T. Goldman and A. Simon, "Theory of Sputtering by High Speed Ions," Phys. Rev. 111, 383 (July 1958).

27. P. Sigmund, "Theory of Sputtering. I. Sputtering Yield of Amorphous and Poiyerystalline Targets," Phys. Rev. 184, 383 (August 1969).

28. Koichi Kanaya, Kiichi Hojou, Kikuo Koga, and Kazuyuki Toki, "Consistent Theory of Sputtering of Solid Targets by Ion Bombardment Using Power Potential Law," Japan. J. App 1. Phys. 12,1297 (September 1963).

29. G. Carter and J. S. Colligan, Ion Bombardment of Solids (American-Elsevier Company, New York, 1968), Chap. 7, pp. 310-353.

30. T. S. Baer and J. N. Anno, "Fast-Neutron Sputtering of Iron at Various Temperatures," $\mathrm{J}$. Nuc1. Mater. 54, 79 (1974).

31. 0. C. Yonts, "Sputtering of Niobium by $\mathrm{D}^{+}$ and $\mathrm{He}^{+}$Ions," Oak Ridge National Laboratory report ORNL-TM-2682 (September 1969).

32. R. S. Nelson, "An Investigation of Thermal Spikes by Studying High Energy Sputtering of Metals at Elevated Temperatures," Philos. Mag., Ser. 8, 11, 291 (1965).

33. B. Feinberg and R. S. Post, "Graphite Surface Erosion and Blistering," J. Vac. Sci. Techno 1. 13, 443 (January/February 1976). 
34. D. Rosenberg and C. K. Wehner, "Sputtering Yields for Low Energy $\mathrm{He}, \mathrm{Kr}$, and Xe Bombardment," J. Appl. Phys. 33, 1842 (May 1962).

35. D. J. Barber, F. C. Frank, M. Moss, and J. W. Steeds, "Prediction of Ion Bombardment Surface Topographies Using Frank's Kinematic Theory of Crystal Dissolution," $J$. Mater. Sci. 8, 1030 (1973).

36. R. P. Edvin, "Measurement of the Sputter Rate of Fused Silica Bombarded by Argon Ions of Energy 12-32 keV," J. Phys. D, Appl. Phys. 6, 833 (1973).

37. A. J. Summers, H. J. Freeman, and N. R. Daly, "Sputtering of Niobium by Niobium, Hydrogen, Deuterium, and Helium Ions in the 10-80 keV Range," J. Appl. Phys. 42, 4774 (1.971).

38. M. Kaminsky, "Plasma Contamination and Wall Erosion in Thermonuclear Reactors," IEEE Trans. Nucl. Sci. NS18, 208 (1971).

39. G. L. Kulcinski, R. W. Conn, G. Lang, L. Wittenberg, D. Sze, J. Kesner, and D. $L$. Kummer, "A Method to Reduce the Effects of Plasma Contamination and First-Wall Erosion in Fusion Reactors," University of Wisconsin Nuclear Engineering Department report UWFDM108 (August 9, 1974; revised September 15, $1974)$.

40. G. K. Wehner, "Sputtering Yields for Normally Incident $\mathrm{Hg}^{+}$-Ion Bombardment at Low Ion Energy," Phys. Rev. 108, 35 (1957).

41. N. Laegreid and G. K. Wehner, "Sputtering Yields of Metals for $\mathrm{Ar}^{+}$and $\mathrm{Ne}^{+}$Ions with Energies from 50 to $600 \mathrm{eV}, " \mathrm{~J}$. Appl. Phys. 32, 365 (March 1961).

42. N. Laegreid and S. D. Dahlgren, "Wa1l Sputtering and Wall Life," J. Appl. Phys. 44, 2093 (May 1973).

43. G. I. Bell and S. Glasstone, Nuclear Reactor Theory (Van Nostrand, New York, T970).

44. A. M. Weinberg and E. P. Wigner, The Physical Theory of Neutron Chain Reactors (Univ. of Chicago Press, Chicago, Illinois, 1968).

45. E. 0. Cashwell and C. J. Everett, A Practical Manual on the Monte Carlo Method for Random Walk Probtems TPergamon Press, New York, T959).

46. L. L. Carter and E. D. Cashwell, "ParticleTransport Simulation with the Monte Carlo
Method," US Energy Research and Development Administration report TID 26607 (1975).

47. I. 0. Bohachersky, "Scaling of Reactor Cavity Wall Loads and Stresses," Los Alanos National Laboratory report LA-7014 -AS (November 1977).

48. T. G. Frank, D. Dudziak, and E. Heck, "Some Neutronic Aspects of Laser Fusion Reactors." Am. Nucl. Soc. Proc. Topical Meeting on the Tech. of Controlled Nuclear Fusion, 1st, San Diego, California, April 16-18, 1974 (National Technical Information Service, US Dept. of Commerce) CONF 74 0402-P1, Vol. 1 . p. 142 .

49. R. J. Roark, Formulas for Stress and Strajn (McGraw-Hi 1), New York, T965).

50. J. D. Achenback, Wave Propagation in Elastic Solids (North Holland, Amsterdam, T973).

51. J. Weertman, "Theory of Irradiation Induced Growth of Fatigue Cracks," J. N. Mater. 55 , 253-258 (1975).

52. I. 0. Bohachersky, L. A. Booth, J. F. Hafer, and J. H. Pendergrass, "Effect of Pellet Yield on Electricity Cost in Laser Fusion Generating Stations," Proc. Am. Nucl. Soc. Topical Meeting on the Tech. of Controlled Nuclear Fusion, 3rd, Santa Fe, NM, May 9-11, 1978, National Technical Information Service, US Department of Commerce), Yol. I, p. 517 .

53. Robert N. Cherdak, "Data Base for Laser Fusion Power Plant Capital Cost Estimation," Burns and Roe Corp. draft report (1980).

54. L. A. Booth and E. M. Leonard, "A Preliminary Assessment of Heavy Ion Fusion," Am. Mucl. Soc. Transactions, Vol. 34, p. 58 (June 1980).

55. L. A. Booth, "Central Station Power Generation by Laser-Driven Fusion," Nuc]. Eng. and Design 24, 263-313 (1973).

56. F. T. Finch, E. A. Kern, and J. M. Williams, "Laser Fusion Power Plant Systems Analysis," Proc. Am. Nucl. Soc. Topical Meeting on the Tech. of Controlled Thermonuclear Fusion. 1st, San Diego, California, Apri1 16-18, 1974 (National Technica) Information Service, US Department of Comerce) CONF 74 0402-P1.

57. E. A. Kern, T. G. Frank, and L. A. Booth, "Systems Analysis of Laser Fusion Power Plants," ANS Transactions 22, 69 (November $1 \xi 75)$. 